\title{
Extended-soft-core baryon-baryon model ESC16. III. $S=-2$ hyperon-hyperon/nucleon interactions
}

\author{
M. M. Nagels \\ Institute of Mathematics, Astrophysics, and Particle Physics University of Nijmegen, Nijmegen, The Netherlands \\ Th. A. Rijken* \\ Institute of Mathematics, Astrophysics, and Particle Physics; University of Nijmegen, Nijmegen, The Netherlands \\ and Nishina Center for Accelerator-Based Science, Institute for Physical and Chemical Research (RIKEN), \\ Wako, Saitama, 351-0198, Japan \\ Y. Yamamoto $\mathbb{1}^{\dagger}$ \\ Nishina Center for Accelerator-Based Science, Institute for Physical and Chemical Research (RIKEN), Wako, Saitama, 351-0198, Japan
}

(Received 18 April 2020; revised 29 August 2020; accepted 30 October 2020; published 23 November 2020)

\begin{abstract}
Background: This is the third of a series of papers on baryon-baryon (BB) interactions, where the baryons are the lowest lying baryon states with spin and parity $J^{P}=(1 / 2)^{+}$. The paper presents the extended-soft-core (ESC) model ESC16 for BB channels with strangeness $S=-2$.

Purpose: The aim is to describe the ingredients of the $S=-2$ ESC16 potentials, to apply these two-body interactions to BB scattering and via $G$-matrix calculations to hypernuclear systems, and to compare with the presently available experimental information.

Methods: The potentials for $S=-2$ are based on the SU(3) extension of the ESC potentials for the strangeness $S=0$ and $S=-1$ sectors, which are fitted to experimental nucleon-nucleon $(N N)$, hyperon-nucleon $(Y N)$, and hyperon-hyperon $(Y Y)$ data. Flavor SU(3) symmetry is broken only 'kinematically' by the masses of the baryons and the mesons. For the $S=-2$ channels almost no experimental scattering data exist, and the information from hypernuclei is also rather limited. Nevertheless, in the fit to the $S=0$ and $S=-1$ sectors information from the Nagara event and the scarce experimental results on the $\Xi N$ cross sections have been used as (mild) constraints to determine the free parameters in the simultaneous fit of the deuteron and the $N N \oplus Y N$ scattering data. Therefore, the potentials for the $S=-2$ sectors are almost completely determined by the fits to the $N N$, $Y N$ data, and SU(3) symmetry.

Results: Various properties of the $S=-2$ potentials are illustrated by giving results for scattering lengths, bound states, phase parameters, and total cross sections. The well-depth $U_{\Xi}$ is calculated and $\Xi N G$-matrix interactions are derived and applied to $\Xi^{-}$-capture reactions. Here, a phenomenological $\Xi N$ interaction is added to describe the experiments. Furthermore, the ESC16 model supplemented with phenomenological SU(3) symmetric gaussian interactions is analyzed, and attractive $\Xi N$ interactions are obtained. Combined with three-body forces derived from the ESC meson-pair vertices and the Fujita-Miyazawa interaction, yields good baryon well depths.

Conclusions: The ESC16 $S=-2$ potentials, with kinematically broken SU(3) symmetry, provide a basis for realistic calculations in nuclear and hypernuclear physics. For a successful description of the well depth's $U_{N}, U_{\Lambda}, U_{\Sigma}$, and $U_{\Xi}$ and hypernuclear $S=-2$ reactions phenomenological additions are needed.
\end{abstract}

DOI: 10.1103/PhysRevC.102.054003

\section{INTRODUCTION}

This paper, the third in a series of papers following Refs. [1,2], henceforth referred to as I and II respectively, presents the results and predictions of the extended-soft-core ESC16 model for low energy baryon-baryon interactions. It constitutes the next phase in the development of the ESC

\footnotetext{
*t.rijken@science.ru.nl

†ys_yamamoto@riken.jp
}

models and is the follow up of the ESC04 models [3-5] and the ESC08a,b models [6] for $S=0,-1,-2$. In Ref. [7] the Nijmegen soft-core one-boson-exchange (OBE) interactions NSC97a-f for baryon-baryon (BB) systems with $S=$ $-2,-3,-4$ were presented.

For the $S=-2$ hyperon-hyperon $(Y Y)$ and hyperonnucleon $(Y N)$ channels hardly any experimental scattering information is available, and also the information from hypernuclei is very limited. Recently the experimental data on double $\Lambda \Lambda$ hypernuclei have very much improved by the observation of the Nagara [8], Hida [9], and Kiso [10] events. These events indicate that the $\Lambda \Lambda$ interaction is rather weak, 
in contrast to the estimates based on the older experimental observations $[11,12]$.

Apart from the experimental activity there is also much interest in the $\Xi N$ interaction from the theoretical side, e.g., (i) chiral field theory $[13,14]$, and (ii) lattice QCD [15].

In the virtual absence of experimental $S=-2$ scattering information, we assume that the potentials obey (broken) flavor SU(3) symmetry, which appears to work very well in the extension from the nucleon-nucleon $(N N)$ to the $S=-1$ hyperon-nucleon $(Y N)$ channels. As in I and II, the potentials are parametrized in terms of meson-baryon-baryon, and meson-pair-baryon-baryon couplings and gaussian form factors as well as diffractive couplings and Pauli blocking. This enables us to include in the interaction one-boson exchange (OBE), two-pseudoscalar exchange (TME), and meson-pair exchange (MPE), and diffractive contributions without any new parameters. All parameters have been fixed by a simultaneous fit to the $N N$ and $Y N$ data, with the constraints imposed (i) for $\Lambda \Lambda\left({ }^{1} S_{0}\right)$ from the Nagara event, (ii) the repulsive well-depth $U_{\Sigma}$, and (iii) the attractive well-depth $U_{\Xi}$. For the procedure see the descriptions in I and II. This way, each $N N \oplus Y N$ model leads to a $Y Y, Y N$ model in a well-defined way, and the predictions for the $\Lambda \Lambda, \Xi N, \Sigma \Lambda$, and $\Sigma \Sigma$ channels contain no additional free parameters. We have chosen for ESC16 the options: SU(3) symmetry for the coupling constants, and pseudovector coupling for the pseudoscalar mesons. (In ESC04 also alternative options were investigated, but it appeared that there is no reason to choose any of these.) Then, SU(3) symmetry allows us to define all coupling constants needed to describe the multistrange interactions in the baryon-baryon channels occurring in $\{8\} \otimes\{8\}$. Quantum chromodynamics (QCD) is, as is generally accepted now, the physical basis of the strong interactions. Since in QCD the gluons are flavor blind, SU(3) symmetry is a basic symmetry, which is broken by the chiral-symmetry breaking at low energies. This picture supports our assumptions, stated already, on SU(3) symmetry. As is shown in [3,4] the coupling constants and the $F /(F+D)$ ratios used in the ESC04 models follow the predictions of the ${ }^{3} P_{0}$-pair creation model (QPC) [16] rather closely. The same is the case for the ESC08 models, see paper I and Ref. [17] for details. Now, it has been shown that in the strong-coupling Hamiltonian lattice formulation of QCD, the flux-tube model, that this is indeed the dominant picture in flux-tube breaking [18]. Therefore, since the ESC models are very much in line with the quark model and QCD, and SU(3) is a good symmetry, the predictions for the $S=-2$ channels can be expected to be realistic.

It is important to study $\Lambda \Lambda$ and $\Xi$ hypernuclei, for understanding of the $\mathrm{BB}$ interactions, giving feedback to the construction of these interactions. This all the more so in view of the at present meager scattering data. An experimental analysis of the $\Xi$-nucleus interaction indicates an attractive nuclear well-depth $U_{\Xi} \approx-14 \mathrm{MeV}$ [19], which is not really small. Therefore, it is very interesting to study the ESC BB interactions in relation to the hypernuclei, using the $G$-matrix $Y N G$ approach, see, e.g., [20].

Since the $G$-matrix studies with ESC potentials extend over quite a period of time, it is appropriate to give here a brief overview for the ESC08 and ESC16 models. In ESC08 models (ESC08a/b/a" [21] and ESC08c [22], 'effective' $\Xi N$ interactions $V_{\text {eff }}$, working mainly in $I=1{ }^{3} S_{1}$ states, have been added in the $G$-matrix calculations such as to give an attractive well-depth $U_{\Xi}$ as indicated in [19]. Then, the $\left({ }^{3} S_{1}, I=1\right)$ contributions for ESC08a/b are extremely attractive, and those for ESC08a"/c are moderate. Even in the latter case, however, they had the notable feature of a (artificial) "deuteron-like" bound state in the $\Xi N\left({ }^{3} S_{1}, I=\right.$ 1) channel, accessible in $K^{-}-K^{+}$-transition $\Xi$-production experiments at JPARC. (This, provided the "effective" interactions are interpreted as genuine two-body interactions.) Also in ESC16, 'effective' $\Xi N$ interactions $V_{\text {eff }}$ are added: the several ESC16 $\oplus V_{\text {eff }}$ models are referred to as ESC16A1, ESC16A2, ESC16B1, and ESC16B2. In ESC16A1/A2, $V_{\text {eff }}$ works mainly in the $I=1{ }^{3} S_{1}$ state as is also the case in ESC08a"/c. In ESCB16B1/B2, $V_{\text {eff }}$ works in $\left({ }^{3} S_{1}, I=1\right)$ and $\left({ }^{1} S_{0}, I=0\right)$ states, in which there is no occurrence of a "deuteron-like bound" state.

The ESC two-body potentials, although very successful for $N N$ and $Y N(S=-1)$, appear to be inadequate for the strangeness $S=-2$ hypernuclei. The nature of $V_{\text {eff }}$, mentioned before, from the viewpoint of SU(3) symmetry is unsatisfactory. In particular the separation of genuine twobody and three-body contributions is unclear. Therefore, extensions of ESC16, called ESC16 ${ }^{\star}$, are studied where phenomenological SU3-symmetric two-body gaussian contact potentials are introduced. These yield good results for $N N$, $Y N, Y Y$, and $\mathrm{BB}$ well depths.

The material in this paper is organized by the following considerations. Most of the details of the SU(3) description are well known. In particular for baryon-baryon scattering the details can be found in papers I, II, and in Refs. [7,23,24]. Here, we restrict ourselves to a minimal exposition of these matters that is necessary for the readability of this paper. Therefore, in Sec. II we first review for $S=-2$ the baryon-baryon multichannel description, and present the SU(3)-symmetric interaction Hamiltonian describing the interaction vertices between mesons and members of the $J^{P}=$ $(1 / 2)^{+}$baryon octet, and define their coupling constants. We then identify the various channels which occur in the $S=-2$ baryon-baryon systems. In Appendix C the potentials on the isospin basis are given in terms of the SU(3) irreps. In most cases, the interaction is a multichannel interaction, characterized by transition potentials and thresholds. Details are given in Refs. [7,23]. For the details on the pair interactions, we refer to papers I and II [1,2]. In Sec. III we give a general treatment of the problem of flavor-exchange forces, which is very helpful to understand the proper treatment of exchange forces and the treatment of baryon-baryon channels with identical particles. Section IV reviews the short-range phenomenology of ESC16 and its implementation in the $S=$ -2 channels. In Sec. $\mathrm{V}$ we describe briefly the treatment of the multichannel thresholds in the potentials. In Sec. VI we present the results of the ESC16 potentials for all the sectors with total strangeness $S=-2$. We give the couplings and $F /(F+D)$ ratios for OBE exchanges of ESC16. Similarly, tables with the pair couplings are shown in Appendix D. We give the $S$-wave scattering lengths, discuss the possibility of bound states in these partial waves. Also, we give the $S$-matrix 
information for the elastic channels in terms of the BryanKlarsfeld-Sprung (BKS) phase parameters [25-27], or in the Kabir-Kermode (KK) [28] format. Tables with the BKS-phase parameters are displayed in Appendix E. Such information is very useful for example for the construction of the $\Lambda-, \Sigma$-, and $\Xi$-nucleus potentials. We also give results for the total cross sections for all leading channels. The scattering cross sections [29-31] indicate that the two-body $\Xi N$ interactions are rather weak, which is also found with the ESC16 model presented in this paper. Therefore, it is expected that there are no $\Xi N$ bound states, in agreement with experimental evidence [32].

In Sec. VII, we present the results of the $\Xi N G$-matrix interactions derived from ESC16 as density-dependent local potentials. Here, structure calculations for $\Xi$ hypernuclei are performed with use of $\Xi$-nucleus folding potentials obtained from the $G$-matrix interactions. In Sec. VIII an $\mathrm{SU}(3)$ symmetric phenomenological addition to ESC16, henceforth referred to as the ESC16* model, is described and results for the $N N \oplus Y N$ fit and the BB well depths are given. We conclude the paper with a summary and final remarks in Sec. IX.

\section{CHANNELS, POTENTIALS, AND SU(3) SYMMETRY}

\section{A. Multichannel formalism}

In this paper we consider the baryon-baryon reactions with $S=-2$ :

$$
A_{1}\left(p_{a}, s_{a}\right)+B_{1}\left(p_{b}, s_{b}\right) \rightarrow A_{2}\left(p_{a}^{\prime}, s_{a}^{\prime}\right)+B_{2}\left(p_{b}^{\prime}, s_{b}^{\prime}\right) .
$$

Like in papers I, II, and Refs. [23,24] we will for the $Y N$ channels also refer to $A_{1}$ and $A_{2}$ as particles 1 and 3 , and to $B_{1}$ and $B_{2}$ as particles 2 and 4 . For the kinematics and the definition of the amplitudes, we refer to paper I [3] of this series. Similar material can be found in Refs. [7,24]. Also, in paper I the derivation of the Lippmann-Schwinger equation in the context of the relativistic two-body equation is described.

The $S=-2$ the BB channels on the particle basis has five systems with different charge. They are, see Refs. [6,7],

$$
\begin{array}{ll}
q=+2: & \Sigma^{+} \Sigma^{+} \rightarrow \Sigma^{+} \Sigma^{+}, \\
q=+1: & \left(\Xi^{0} p, \Sigma^{+} \Lambda, \Sigma^{0} \Sigma^{+}\right) \rightarrow\left(\Xi^{0} p, \Sigma^{+} \Lambda, \Sigma^{0} \Sigma^{+}\right), \\
q=0: & \left(\Lambda \Lambda, \Xi^{0} n, \Xi^{-} p, \Sigma^{0} \Lambda, \Sigma^{0} \Sigma^{0}, \Sigma^{-} \Sigma^{+}\right) \\
& \rightarrow\left(\Lambda \Lambda, \Xi^{0} n, \Xi^{-} p, \Sigma^{0} \Lambda, \Sigma^{0} \Sigma^{0}, \Sigma^{-} \Sigma^{+}\right), \\
q=-1: & \left(\Xi^{-} n, \Sigma^{-} \Lambda, \Sigma^{-} \Sigma^{0}\right) \rightarrow\left(\Xi^{-} n, \Sigma^{-} \Lambda, \Sigma^{-} \Sigma^{0}\right), \\
q=-2: & \Sigma^{-} \Sigma^{-} \rightarrow \Sigma^{-} \Sigma^{-} .
\end{array}
$$

Like in Refs. [23,24], the potentials are calculated on the isospin basis. For $S=-2$ hyperon-nucleon systems there are three isospin channels:

$$
\begin{aligned}
& I=0:(\Lambda \Lambda, \Xi N, \Sigma \Sigma \rightarrow \Lambda \Lambda, \Xi N, \Sigma \Sigma), \\
& I=1:(\Xi N, \Sigma \Lambda, \Sigma \Sigma \rightarrow \Xi N, \Sigma \Lambda, \Sigma \Sigma), \\
& I=2: \Sigma \Sigma \rightarrow \Sigma \Sigma .
\end{aligned}
$$

The potential on the particle basis for the $q=2$ and $q=-2$ channels are given by the $I=2 \Sigma \Sigma$ potential on the isospin basis. For $q=0$ and $q= \pm 1$, the potentials are related to the potentials on the isospin basis by an isospin rotation. The connection of the potentials on the particle and the isospin bases are explicitly given in [6] and given in Appendix A for completeness.

For the kinematics of the reactions and the various thresholds, see Ref. [23], and Sec. V. In this work we do not solve the Lippmann-Schwinger equation, but the multichannel Schrödinger equation in configuration space, completely analogous to [24]. The multichannel Schrödinger equation for the configuration-space potential is derived from the LippmannSchwinger equation through the standard Fourier transform, and the equation for the radial wave function is found to be of the form in paper II and Ref. [24]:

$$
u_{l, j}^{\prime \prime}+\left(p_{i}^{2} \delta_{i, j}-A_{i, j}\right) u_{l, j}-B_{i, j} u_{l, j}^{\prime}=0,
$$

where $A_{i, j}$ contains the potential, nonlocal contributions, and the centrifugal barrier, while $B_{i, j}$ is only present when nonlocal contributions are included. The solution in the presence of open and closed channels is given, for example, in Ref. [33]. The inclusion of the Coulomb interaction in the configurationspace equation is well known and included in the evaluation of the scattering matrix.

The momentum space and configuration space potentials for the ESC model have been described in Ref. [3] for baryonbaryon in general. Therefore, they apply also to the $S=-2$ hyperon-hyperon/nucleon channels and we can refer for that part of the potential to papers I and II. Also in the ESC model, the potentials are of such a form that they are exactly equivalent in both momentum space and configuration space. The treatment of the mass differences among the baryons are handled exactly similar as is done in [23,24]. Also, exchange potentials related to strange meson exchanges $K, K^{*}$, etc., are given in paper II [2].

The baryon mass differences in the intermediate states for TME and MPE potentials have been neglected, just like in $S=-1 Y N$ scattering. This, although possible in principle, becomes rather laborious and is not expected to change the characteristics of the baryon-baryon potentials.

\section{B. Potentials and SU(3) symmetry}

Like in all Nijmegen BB-interaction models we consider all $\mathrm{BB}$ channels, where the baryons are the members of the $J^{P}=\frac{1}{2}^{+}$baryon octet

$$
B=\left(\begin{array}{ccc}
\frac{\Sigma^{0}}{\sqrt{2}}+\frac{\Lambda}{\sqrt{6}} & \Sigma^{+} & p \\
\Sigma^{-} & -\frac{\Sigma^{0}}{\sqrt{2}}+\frac{\Lambda}{\sqrt{6}} & n \\
-\Xi^{-} & \Xi^{0} & -\frac{2 \Lambda}{\sqrt{6}}
\end{array}\right) .
$$

The baryon masses, used in this paper, are given in Table V.

Similar to Eq. (2.5) the nonets $M(9)$ for the pseudoscalar, vector, scalar, and axial-vector mesons can be represented by (i) the traceless matrices $M(8)=\sum_{i=1}^{8} M_{i} \lambda_{i} / \sqrt{2}$, and (ii) the unitary singlet mesons $M(0)$ are included via the definition

$$
M_{\beta}^{\alpha}(9)=M_{\beta}^{\alpha}(8)+\delta_{\beta}^{\alpha} M(0) / \sqrt{3} .
$$

For the assignment of the meson fields $M_{i}$ and the Gell-Mann matrices $\lambda_{i}$ see Refs. [34,35]. Taking the pseudoscalar mesons with $J^{P}=0^{-}$as a specific example, the nonet is written as $P(9)=P(8)+P(0)$, where the singlet matrix $P(0)$ has 
$\eta_{0} / \sqrt{3}$ on the diagonal, and the octet matrix $P(8)$ is given explicitly by

$$
P(8)=\left(\begin{array}{ccc}
\frac{\pi^{0}}{\sqrt{2}}+\frac{\eta_{8}}{\sqrt{6}} & \pi^{+} & K^{+} \\
\pi^{-} & -\frac{\pi^{0}}{\sqrt{2}}+\frac{\eta_{8}}{\sqrt{6}} & K^{0} \\
K^{-} & \frac{K^{0}}{\sqrt{6}} & -\frac{2 \eta_{8}}{\sqrt{6}}
\end{array}\right) .
$$

Here, $\eta_{8}$ and $\eta_{0}$ are the 'bare' SU(3) octet and singlet state, respectively, and the physical $\eta(448), \eta^{\prime}(958)$ are mixtures of the bare ones.
Using the isodoublets,

$$
\begin{aligned}
& N=\left(\begin{array}{l}
p \\
n
\end{array}\right), \quad \Xi=\left(\begin{array}{c}
\Xi^{0} \\
\Xi^{-}
\end{array}\right), \\
& K=\left(\begin{array}{l}
K^{+} \\
K^{0}
\end{array}\right), \quad K_{c}=\left(\begin{array}{c}
\overline{K^{0}} \\
-K^{-}
\end{array}\right),
\end{aligned}
$$

the SU(3) invariant interaction Hamiltonian for pseudoscalar mesons, omitting the Lorentz structure, reads, given by the interaction Hamiltonian [34,35],

$$
\begin{aligned}
\mathcal{H}_{\mathrm{pv}}^{\mathrm{oct}}= & \left\{f_{N N \pi}(\bar{N} \boldsymbol{\tau} N) \boldsymbol{\pi}-i f_{\Sigma \Sigma \pi}(\overline{\boldsymbol{\Sigma}} \times \boldsymbol{\Sigma}) \boldsymbol{\pi}+f_{\Lambda \Sigma \pi}(\bar{\Lambda} \boldsymbol{\Sigma}+\overline{\boldsymbol{\Sigma}} \Lambda) \boldsymbol{\pi}+f_{\Xi \Xi \pi}(\bar{\Xi} \boldsymbol{\tau} \Xi) \boldsymbol{\pi}+f_{\Lambda N K}[(\bar{N} K) \Lambda+\bar{\Lambda}(\bar{K} N)]\right. \\
& +f_{\Xi \Lambda K}\left[\left(\bar{\Xi} K_{c}\right) \Lambda+\bar{\Lambda}\left(\overline{K_{c}} \boldsymbol{\Xi}\right)\right]+f_{\Sigma N K}[\overline{\boldsymbol{\Sigma}}(\bar{K} \boldsymbol{\tau} N)+(\bar{N} \boldsymbol{\tau} K) \boldsymbol{\Sigma}]+f_{\Xi \Sigma K}\left[\overline{\boldsymbol{\Sigma}}\left(\overline{K_{c}} \boldsymbol{\tau} \Xi\right)+\left(\bar{\Xi} \boldsymbol{\tau} K_{c}\right) \boldsymbol{\Sigma}\right] \\
& +f_{N N \eta_{8}}(\bar{N} N) \eta_{8}+f_{\Lambda \Lambda \eta_{8}}(\bar{\Lambda} \Lambda) \eta_{8}+f_{\Sigma \Sigma \eta_{8}}(\overline{\boldsymbol{\Sigma}} \boldsymbol{\Sigma}) \eta_{8}+f_{\Xi \Xi \eta_{8}}(\bar{\Xi} \Xi) \eta_{8} \\
& \left.+f_{N N \eta_{0}}(\bar{N} N) \eta_{0}+f_{\Lambda \Lambda \eta_{0}}(\bar{\Lambda} \Lambda) \eta_{0}+f_{\Sigma \Sigma \eta_{0}}(\overline{\boldsymbol{\Sigma}} \boldsymbol{\Sigma}) \eta_{0}+f_{\Xi \Xi \eta_{0}}(\bar{\Xi} \Xi) \eta_{0}\right\} / m_{\pi} .
\end{aligned}
$$

For the other mesons the octet matrix is obtained by the following substitutions: (i) vector mesons $\pi \rightarrow \rho, \eta_{8} \rightarrow \phi_{8}$, $K \rightarrow K^{*}$, (ii) scalar $\pi \rightarrow a_{0}, \eta_{8} \rightarrow f_{0,8}, K \rightarrow \kappa$, (iii) axialvector $\pi \rightarrow A_{1}, \eta_{8} \rightarrow e_{8}, K \rightarrow K_{1 A}$, (iv) axial-vector $\pi \rightarrow$ $B_{1}, \eta_{8} \rightarrow h_{8}, K \rightarrow K_{1 B}$.

All coupling constants for the physical mesons can be expressed in terms of three SU(3) parameters: (i) singlet and octet couplings $\left(f_{1}, f_{8}\right)$, respectively, and (ii) the $F /(F+D)$ ratio $\alpha_{P}$. For details see, e.g., Refs. [23,34]. The same applies to the nonets of the vector-, scalar-, and axial-vector mesons. The Pomeron is treated as an SU(3) singlet.

In Appendix C, Tables XXVI and XXVII, we give the relation between the potentials on the isospin basis, see Eqs. (A1) and (A2), and the SU(3) irreps.

Given the interaction Hamiltonian (2.9) and a theoretical scheme for deriving the potential representing a particular Feynman diagram, it is now straightforward to derive the one-meson-exchange baryon-baryon potentials. We follow the Thompson approach [36-40] and expressions for the potential in momentum space can be found in paper I, as well as configuration space potentials. Since the nucleons have strangeness $S=0$, the hyperons $S=-1$, and the cascades $S=-2$, the possible baryon-baryon interaction channels can be classified according to their total strangeness, ranging from $S=0$ for $N N$ to $S=-4$ for $\Xi \Xi$. Apart from the wealth of accurate $N N$ scattering data for the total strangeness $S=0$ sector, there are only a few, and not very accurate, $Y N$ scattering data for the $S=-1$ sector, while there are no data at all for the $S<-1$ sectors. We therefore believe that at this stage it is not yet worthwhile to explicitly account for the small mass differences between the specific charge states of the baryons and mesons; i.e., we use average masses, isospin is a good quantum number, and the potentials are calculated on the isospin basis. The possible channels on the isospin basis are given in Eq. (2.3).

However, the Lippmann-Schwinger or Schrödinger equation is solved for the physical particle channels, and so scattering observables are calculated using the proper physical baryon masses. The possible channels on the physical particle basis can be classified according to the total charge $Q$; these are given in Eq. (2.2). The corresponding potentials are obtained from the potential on the isospin basis by making the appropriate isospin rotations. The matrix elements of the isospin rotation matrices are nothing else but the ClebschGordan coefficients for the two baryon isospins making up the total isospin. (Note that this is the reason why the potential on the particle basis, obtained from applying an isospin rotation to the potential on the isospin basis, will have the correct sign for any coupling constant on a vertex which involves a $\Sigma^{+}$.)

In order to construct the potentials on the isospin basis, we need first the matrix elements of the various OBE exchanges between particular isospin states. Using the isomultiplets (2.7) and the Hamiltonian (2.9) the isospin factors can be calculated. The results are given in Table I, where we use the pseudoscalar mesons as a specific example. The entries contain the flavor-exchange operator $P_{f}$, which is +1 for a flavor symmetric and -1 for a flavor antisymmetric two-baryon state. Since two-baryon states are totally antisymmetric, one has $P_{f}=-P_{x} P_{\sigma}$. Therefore, the exchange operator $P_{f}$ has the value $P_{f}=+1$ for even- $L$ singlet and odd- $L$ triplet partial waves, and $P_{f}=-1$ for odd- $L$ singlet and even- $L$ triplet partial waves. In order to understand Table I fully, we have given in Sec. III a general treatment of exchange forces. This treatment shows also how to deal with the case where the initial/final state involves identical particles and the final/initial state does not.

Secondly, we need to evaluate the TME and the MPE exchanges. The method we used for these is the same as for hyperon-nucleon, and is described in Ref. [4], Sec. II D.

\section{EXCHANGE FORCES}

The proper treatment of the flavor-exchange forces for the $S=-2$ channels is a little more subtle than for the 
TABLE I. Meson-exchange isospin factors for the $Y N$ and $Y Y$ elastic and inelastic channels with strangeness $S=-2$ and isospin I. $P_{f}$ is the flavor-exchange operator. The $I=2$ occurs only in the $S=-2 \Sigma \Sigma$ channels, where the isospin factors are given by $\left(\Sigma \Sigma\left|\eta, \eta^{\prime}, \pi\right| \Sigma \Sigma\right)=\frac{1}{2}\left(1+P_{f}\right)$.

\begin{tabular}{lcc}
\hline \hline$S=-2$ & $I=0$ & $I=1$ \\
\hline$\left(\Lambda \Lambda\left|\eta, \eta^{\prime}\right| \Lambda \Lambda\right)$ & $\frac{1}{2}\left(1+P_{f}\right)$ & - \\
$\left(\Xi N\left|\eta, \eta^{\prime}\right| \Xi N\right)$ & 1 & 1 \\
$\left(\Sigma \Sigma\left|\eta, \eta^{\prime}\right| \Sigma \Sigma\right)$ & $\frac{1}{2}\left(1+P_{f}\right)$ & $\frac{1}{2}\left(1-P_{f}\right)$ \\
$\left(\Sigma \Lambda\left|\eta, \eta^{\prime}\right| \Sigma \Lambda\right)$ & - & 1 \\
$(\Xi N|\pi| \Xi N)$ & -3 & 1 \\
$(\Sigma \Sigma|\pi| \Sigma \Sigma)$ & $-\left(1+P_{f}\right)$ & $-\frac{1}{2}\left(1-P_{f}\right)$ \\
$(\Lambda \Lambda|\pi| \Sigma \Sigma)$ & $-\frac{1}{2} \sqrt{3}\left(1+P_{f}\right)$ & - \\
$(\Sigma \Lambda|\pi| \Lambda \Sigma)$ & - & $P_{f}$ \\
$(\Sigma \Sigma|\pi| \Sigma \Lambda)$ & - & - \\
$(\Lambda \Lambda|K| \Xi N)$ & $1+P_{f}$ & $\sqrt{2}\left(1-P_{f}\right)$ \\
$(\Sigma \Sigma|K| \Xi N)$ & $\sqrt{3}\left(1+P_{f}\right)$ & $\sqrt{2} ;-P_{f} \sqrt{2}$ \\
$(\Xi N|K| \Sigma \Lambda)$ & - & \\
\hline \hline
\end{tabular}

$S=0,-1,-3,-4$ channels. The extra complication is the occurrence of couplings between channels with identical and channels with nonidentical particles. In order to understand the several $\sqrt{2}$ factors, mentioned in Ref. [7] and treated in a concise form in Ref. [6], we give a systematic treatment of the flavor-exchange potentials. The method followed is using a multichannel framework, which starts by ordering the twoparticle states by assigning $A_{i}$ and $B_{i}$ for the channel labeled with the index $i$, like in Eq. (2.1). The particles $A_{i}$ and $B_{i}$ have the center-of-mass (c.m.) momenta $p_{i}$ and $-p_{i}$, spin components $s_{A, i}$ and $s_{B, i}$. The two-baryon states $\left|A_{i} B_{i}\right\rangle$ and $\left|B_{i} A_{i}\right\rangle$ are considered to be distinct, leading to distinct two-baryon channels. The 'direct' and the 'exchange' $T$ amplitudes are given by the $T$-matrix elements

$$
\left\langle A_{j} B_{j}\left|T_{d}\right| A_{i} B_{i}\right\rangle, \quad\left\langle B_{j} A_{j}\left|T_{e}\right| A_{i} B_{i}\right\rangle,
$$

and similarly for the direct and flavor-exchange potentials $V_{d}$ and $V_{e}$. It is obvious from rotation invariance that

$$
\begin{aligned}
\left\langle A_{j} B_{j}\left|T_{d}\right| A_{i} B_{i}\right\rangle & =\left\langle B_{j} A_{j}\left|T_{d}\right| B_{i} A_{i}\right\rangle, \\
\left\langle B_{j} A_{j}\left|T_{e}\right| A_{i} B_{i}\right\rangle & =\left\langle A_{j} B_{j}\left|T_{e}\right| B_{i} A_{i}\right\rangle .
\end{aligned}
$$

A similar definition (3.1) and relation (3.2) apply for the direct and flavor-exchange potentials $V_{d}$ and $V_{e}$. We notice that in interchanging $A$ and $B$ there is no exchange of momenta or spin components, see Fig. 1. This is necessary for the application of Lippmann-Schwinger type of integral equations, which can produce only one type of the Mandelstam double spectral functions [41], e.g., $\rho(s, t)$ or $\rho(s, u)$. [The third double spectral function $\rho(t, u)$ can only be included approximately in potential scattering.] So, the momentum transfer for $V_{d}$ and for $V_{e}$ is the same. Viewed from the coupled-channel scheme this is the standard situation.

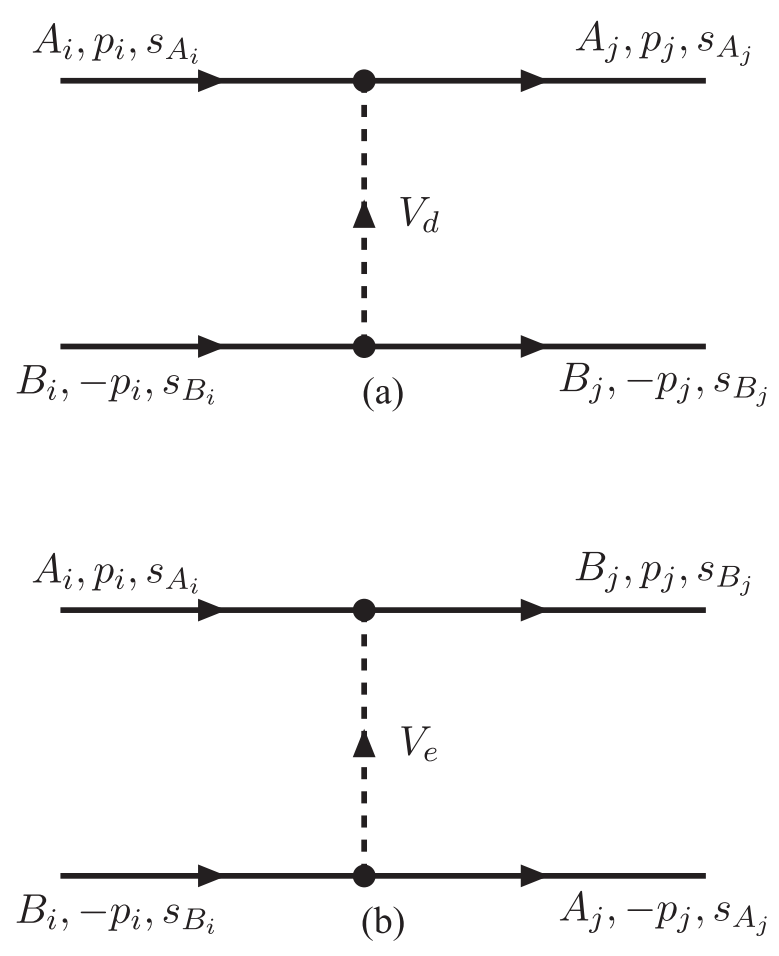

FIG. 1. $V_{d}$ (a) and $V_{e}$ (b) in the c.m. system.

The integral equations with two-baryon unitarity, e.g., the Thompson, Lippmann-Schwinger equation, etc., read for the $T_{d}$ and $T_{e}$ operators

$$
\begin{aligned}
\left\langle A_{j} B_{j}\left|T_{d}\right| A_{i} B_{i}\right\rangle= & \left\langle A_{j} B_{j}\left|V_{d}\right| A_{i} B_{i}\right\rangle \\
& +\sum_{k}\left[\left\langle A_{j} B_{j}\left|V_{d}\right| A_{k} B_{k}\right\rangle G_{k}\left\langle A_{k} B_{k}\left|T_{d}\right| A_{i} B_{i}\right\rangle\right. \\
& \left.+\left\langle A_{j} B_{j}\left|V_{e}\right| B_{k} A_{k}\right\rangle G_{k}\left\langle B_{k} A_{k}\left|T_{e}\right| A_{i} B_{i}\right\rangle\right], \\
\left\langle B_{j} A_{j}\left|T_{e}\right| A_{i} B_{i}\right\rangle= & \left\langle B_{j} A_{j}\left|V_{e}\right| A_{i} B_{i}\right\rangle \\
& +\sum_{k}\left[\left\langle B_{j} A_{j}\left|V_{d}\right| B_{k} A_{k}\right\rangle G_{k}\left\langle B_{k} A_{k}\left|T_{e}\right| A_{i} B_{i}\right\rangle\right. \\
& \left.+\left\langle B_{j} A_{j}\left|V_{e}\right| A_{k} B_{k}\right\rangle G_{k}\left\langle A_{k} B_{k}\left|T_{d}\right| A_{i} B_{i}\right\rangle\right] .
\end{aligned}
$$

These coupled equations can be diagonalized by introducing the $T^{ \pm}$and $V^{ \pm}$operators

$$
T^{ \pm}=T_{d} \pm T_{e}, \quad V^{ \pm}=V_{d} \pm V_{e},
$$

which, as follows from Eq. (3.3), satisfy separate integral equations

$$
\begin{aligned}
\left\langle A_{j} B_{j}\left|T^{ \pm}\right| A_{i} B_{i}\right\rangle= & \left\langle A_{j} B_{j}\left|V^{ \pm}\right| A_{i} B_{i}\right\rangle+\sum_{k}\left\langle A_{j} B_{j}\left|V^{ \pm}\right| A_{k} B_{k}\right\rangle \\
& \times G_{k}\left\langle A_{k} B_{k}\left|T^{ \pm}\right| A_{i} B_{i}\right\rangle
\end{aligned}
$$


Notice that on the basis of states with definite flavor symmetry

$$
\left|A_{i} B_{i}\right\rangle_{ \pm}=\frac{1}{\sqrt{2}}\left[\left|A_{i} B_{i}\right\rangle \pm\left|B_{i} A_{i}\right\rangle\right]
$$

the $T^{ \pm}$and $V^{ \pm}$matrix elements are also given by

$$
T_{i j}^{ \pm}={ }_{ \pm}\left\langle A_{i} B_{i}|T| A_{j} B_{j}\right\rangle_{ \pm}, \quad V_{i j}^{ \pm}={ }_{ \pm}\left\langle A_{i} B_{i}|V| A_{j} B_{j}\right\rangle_{ \pm} .
$$

\section{A. Identical particles}

So far, we considered the general case where $A_{i} \neq B_{i}$ for all channels. In the case that $A_{i}=B_{i}$ for some $i$, one has $\left\langle B_{i} A_{i}\left|V_{e}\right| A_{i} B_{i}\right\rangle=0$, because there is no distinct physical state corresponding to the 'flavor exchange-state'. For example for a flavor single channel like $p p$ one deduces from Eq. (3.3) that then also $T_{e}=0$, and one has in this case the integral equation

$$
\begin{aligned}
\left\langle A_{j} B_{j}\left|T_{d}\right| A_{i} B_{i}\right\rangle= & \left\langle A_{j} B_{j}\left|V_{d}\right| A_{i} B_{i}\right\rangle+\sum_{k}\left\langle A_{j} B_{j}\left|V_{d}\right| A_{k} B_{k}\right\rangle \\
& \times G_{k}\left\langle A_{k} B_{k}\left|T_{d}\right| A_{i} B_{i}\right\rangle
\end{aligned}
$$

where the labels $i$ and $j$ now denote, e.g., the spin components.

\section{B. Coupled $\Lambda \Lambda$ and $\Xi N$ system}

This multichannel system represents the case where there is mixture of channels with identical and with nonidentical particles. The three states we distinguish are $|\Lambda \Lambda\rangle,|\Xi N\rangle$, and $|N \Xi\rangle$. Choosing the same ordering, the potential written as a $3 \times 3$ matrix reads

$$
V=\left(\begin{array}{ccc}
\langle\Lambda \Lambda|V| \Lambda \Lambda\rangle & \langle\Lambda \Lambda|V| \Xi N\rangle & \langle\Lambda \Lambda|V| N \Xi\rangle \\
\langle\Xi N|V| \Lambda \Lambda\rangle & \langle\Xi N|V| \Xi N\rangle & \langle\Xi N|V| N \Xi\rangle \\
\langle N \Xi|V| \Lambda \Lambda\rangle & \langle N \Xi|V| \Xi N\rangle & \langle N \Xi|V| N \Xi\rangle
\end{array}\right)
$$

With a similar notation for the $T$ matrix, the LippmannSchwinger equation can be written compactly as a $3 \times$ 3-matrix equation:

$$
T=V+V G T \text { with } G_{i j}=G_{i} \delta_{i j} .
$$

Next, we make a transformation to states, which are either symmetric or antisymmetric for particle interchange. Then, according to Eq. (3.7), we can separate them in the LippmannSchwinger equation. This is achieved by the transformation

$$
\left(\begin{array}{c}
\Lambda \Lambda \\
\Xi N \\
N \Xi
\end{array}\right) \Rightarrow\left(\begin{array}{c}
\Lambda \Lambda \\
(\Xi N+N \Xi) / \sqrt{2} \\
(\Xi N-N \Xi) / \sqrt{2}
\end{array}\right)
$$

where a standard multichannel notation is used for the states. This yields in the transformed basis the potential

$$
U V U^{-1}=\left(\begin{array}{ccc}
V_{\Lambda \Lambda ; \Lambda \Lambda} & \left(V_{\Lambda \Lambda ; \Xi N}+V_{\Lambda \Lambda ; N \Xi}\right) / \sqrt{2} & \left(V_{\Lambda \Lambda ; \Xi N}-V_{\Lambda \Lambda ; N \Xi}\right) / \sqrt{2} \\
\left(V_{\Xi N ; \Lambda \Lambda}+V_{N \Xi ; \Lambda \Lambda}\right) / \sqrt{2} & \left(V_{\Xi N ; \Xi N}+V_{\Xi N ; N \Xi}\right) & 0 \\
\left(V_{\Xi N ; \Lambda \Lambda}-V_{N \Xi ; \Lambda \Lambda}\right) / \sqrt{2} & 0 & \left(V_{\Xi N ; \Xi N}-V_{\Xi N ; N \Xi}\right)
\end{array}\right),
$$

and of course, a similar form is obtained for the $T$ matrix on the transformed basis. Now, obviously we have that $V_{\Lambda \Lambda ; \Xi N}=$ $V_{\Lambda \Lambda ; N \Xi}$ and $V_{\Xi N ; \Lambda \Lambda}=V_{N \Xi ; \Lambda \Lambda}$. Therefore, one sees that the even and odd states under particle exchange are decoupled in Eq. (3.12). Also $\left(V_{\Xi N ; \Lambda \Lambda}+V_{N \Xi ; \Lambda \Lambda}\right) / \sqrt{2}=\sqrt{2} V_{\Xi N ; \Lambda \Lambda}$, etc., showing the appearance of the $\sqrt{2}$ factors, mentioned before. Indeed, they appear in a systematic way using the multichannel framework. In Ref. [6] the details are worked out for $K-, \eta-$, and $\pi$-exchange potentials for the $\Lambda \Lambda, \Xi N, \Sigma \Lambda$, etc., channels. The results are summarized in Table I.

\section{SHORT-RANGE PHENOMENOLOGY}

For a detailed discussion and description of the short-range region we refer to paper II, Sec. V [2]. Here, the meson and diffractive exchanges and the quark core in the ESC16 modeling have been described. In this section we give the quark-core phenomenology for the $S=-2$ baryon-baryon channels.

\section{A. Relation $S=-2 Y N, Y Y$ states and $\mathrm{SU}_{f s}(6)$ irreps}

The relation between the $\mathrm{SU}_{f}(3)$ irreps and $\mathrm{SU}_{f s}(6)$ irreps, where $f$ and $s$ denote flavor and spin, respectively, has been derived in paper II [2]. In Appendix C the $S=-2$ BB potentials are given in terms of the $\mathrm{SU}(3)_{f}$ irreps. Combining these two things gives the representation of the $S=-2$ potentials in terms of the $\mathrm{SU}_{f s}(6)$ irreps as displayed in Tables II and III.

\section{B. Parametrization quark-core effects}

As introduced in paper II, the repulsive short-range Pomeron-like $Y N, Y Y$ potential is split linearly in a diffractive (Pomeron) and a quark-core component by writing

$$
V_{P B B}=V_{B B}(\mathrm{POM})+V_{B B}(\mathrm{~PB}),
$$

TABLE II. SU $(6)_{f s}$ contents spin-space odd ${ }^{1} S_{0},{ }^{3} P,{ }^{1} D_{2}, \ldots$ potentials on the spin-isospin basis.

\begin{tabular}{lcc}
\hline \hline & $(S, I)$ & $V=a V_{[51]}+b V_{[33]}$ \\
\hline$\Lambda \Lambda \rightarrow \Lambda \Lambda$ & $(0,0)$ & $V_{\Lambda \Lambda, \Lambda \Lambda}=\frac{1}{2} V_{[51]}+\frac{1}{2} V_{[33]}$ \\
$\Xi N \rightarrow \Xi N$ & $(0,0)$ & $V_{\Xi N, \Xi N}=\frac{1}{3} V_{[51]}+\frac{2}{3} V_{[33]}$ \\
$\Sigma \Sigma \rightarrow \Sigma \Sigma$ & $(0,0)$ & $V_{\Sigma \Sigma, \Sigma \Sigma}=\frac{11}{18} V_{[51]}+\frac{7}{18} V_{[33]}$ \\
$\Xi N \rightarrow \Xi N$ & $(0,1)$ & $V_{\Xi N, \Xi N}=\frac{7}{9} V_{[51]}+\frac{2}{9} V_{[33]}$ \\
$\Sigma \Lambda \rightarrow \Sigma \Lambda$ & $(0,1)$ & $V_{\Sigma \Lambda, \Sigma \Lambda}=\frac{2}{3} V_{[51]}+\frac{1}{3} V_{[33]}$ \\
$\Sigma \Sigma \rightarrow \Sigma \Sigma$ & $(0,2)$ & $V_{\Sigma \Sigma, \Sigma \Sigma}=\frac{4}{9} V_{[51]}+\frac{5}{9} V_{[33]}$ \\
\hline \hline
\end{tabular}


TABLE III. SU $(6)_{f s}$-contents of the spin-space even ${ }^{3} S_{1},{ }^{1} P_{1},{ }^{3} D, \ldots$ potentials on the spin-isospin basis.

\begin{tabular}{lcc}
\hline \hline & $(S, I)$ & $V=a V_{[51]}+b V_{[33]}$ \\
\hline$\Xi N \rightarrow \Xi N$ & $(1,0)$ & $V_{\Xi N, \Xi N}=\frac{5}{9} V_{[51]}+\frac{4}{9} V_{[33]}$ \\
$\Xi N \rightarrow \Xi N$ & $(1,1)$ & $V_{\Xi N, \Xi N}=\frac{17}{27} V_{[51]}+\frac{10}{27} V_{[33]}$ \\
$\Sigma \Lambda \rightarrow \Sigma \Lambda$ & $(1,1)$ & $V_{\Sigma \Lambda, \Sigma \Lambda}=\frac{2}{3} V_{[51]}+\frac{1}{3} V_{[33]}$ \\
$\Sigma \Sigma \rightarrow \Sigma \Sigma$ & $(1,1)$ & $V_{\Sigma \Sigma, \Sigma \Sigma}=\frac{16}{27} V_{[51]}+\frac{11}{27} V_{[33]}$ \\
\hline \hline
\end{tabular}

where $V_{B B}(\mathrm{POM})$ represents the genuine Pomeron and $V_{B B}(\mathrm{~PB})$ the structural effects of the quark-core forbidden [51] configuration, i.e., a Pauli-blocking (PB) effect. Since the Pomeron is a unitary singlet its contribution is the same for all BB channels (apart from some small baryon mass breaking effects), i.e., $V_{B B}(\mathrm{POM})=V_{N N}(\mathrm{POM})$. Furthermore the $\mathrm{PB}$-effect for the $\mathrm{BB}$ channels is assumed to be proportional to the relative weight of the forbidden [51] configuration compared to its weight in $N N$

$$
V_{B B}(\mathrm{~PB})=a_{\mathrm{PB}}\left(w_{B B}[51] / w_{N N}[51]\right) V_{N N}(\mathrm{~PB}),
$$

where $a_{\mathrm{PB}}$ denotes the quark-core fraction with respect to the Pomeron potential for the $N N$ channel, i.e., $V_{N N}(\mathrm{~PB})=$ $a_{\mathrm{PB}} V_{P N N}$. Then we have

$$
V_{P B B}=\left(1-a_{\mathrm{PB}}\right) V_{P N N}+a_{P B}\left(\frac{w_{B B}[51]}{w_{N N}[51]}\right) V_{P N N} .
$$

A subtle treatment of all $\mathrm{BB}$ channels according to this linear scheme is characteristic for the ESC16 model. The value of the PB factor $a_{P B}$ is searched in the fit to the $N N$ and $Y N$ data. The parameter $a_{\mathrm{PB}}$ turns out to be about $39 \%$. This means that the quark-core repulsion is roughly $64 \%$ of the genuine Pomeron repulsion. Then, the PB effects in the $S=$ -2 channels are entirely determined. From Eq. (4.3) the ratio $V_{P B B} / V_{P N N}$ is given by the weights of the [51] irrep and $a_{\mathrm{PB}}$. In Table IV we give this ratio for the various $S=-2$ BB channels in the ESC16 model, With only one exception, the effective Pomeron repulsion is stronger than in the $N N$ channels.

TABLE IV. Effective Pomeron $+\mathrm{PB}$ contribution on the spin, isospin basis.

\begin{tabular}{lccc}
\hline \hline & $(S, I)$ & $V_{P B B} / V_{P N N}$ & $\mathrm{ESC16}$ \\
\hline$N N \rightarrow N N$ & $(0,1)$ & 1 & 1.000 \\
$N N \rightarrow N N$ & $(1,0)$ & 1 & 1.000 \\
$\Lambda \Lambda \rightarrow \Lambda \Lambda$ & $(0,0)$ & $1+\frac{1}{8} a_{\mathrm{PB}}$ & 1.049 \\
$\Xi N \rightarrow \Xi N$ & $(0,0)$ & $1-\frac{1}{4} a_{\mathrm{PB}}$ & 0.903 \\
$\Sigma \Sigma \rightarrow \Sigma \Sigma$ & $(0,0)$ & $1+\frac{3}{8} a_{\mathrm{PB}}$ & 1.146 \\
$\Xi N \rightarrow \Xi N$ & $(0,1)$ & $1+\frac{3}{4} a_{\mathrm{PB}}$ & 1.293 \\
$\Sigma \Lambda \rightarrow \Sigma \Lambda$ & $(0,1)$ & $1+\frac{1}{2} a_{\mathrm{PB}}$ & 1.195 \\
$\Sigma \Sigma \rightarrow \Sigma \Sigma$ & $(0,2)$ & 1 & 1.000 \\
$\Xi N \rightarrow \Xi N$ & $(1,0)$ & $1+\frac{1}{4} a_{\mathrm{PB}}$ & 1.098 \\
$\Xi N \rightarrow \Xi N$ & $(1,1)$ & $1+\frac{5}{12} a_{\mathrm{PB}}$ & 1.163 \\
$\Sigma \Lambda \rightarrow \Sigma \Lambda$ & $(1,1)$ & $1+\frac{1}{2} a_{\mathrm{PB}}$ & 1.195 \\
$\Sigma \Sigma \rightarrow \Sigma \Sigma$ & $(1,1)$ & $1+\frac{1}{3} a_{\mathrm{PB}}$ & 1.130 \\
\hline \hline
\end{tabular}

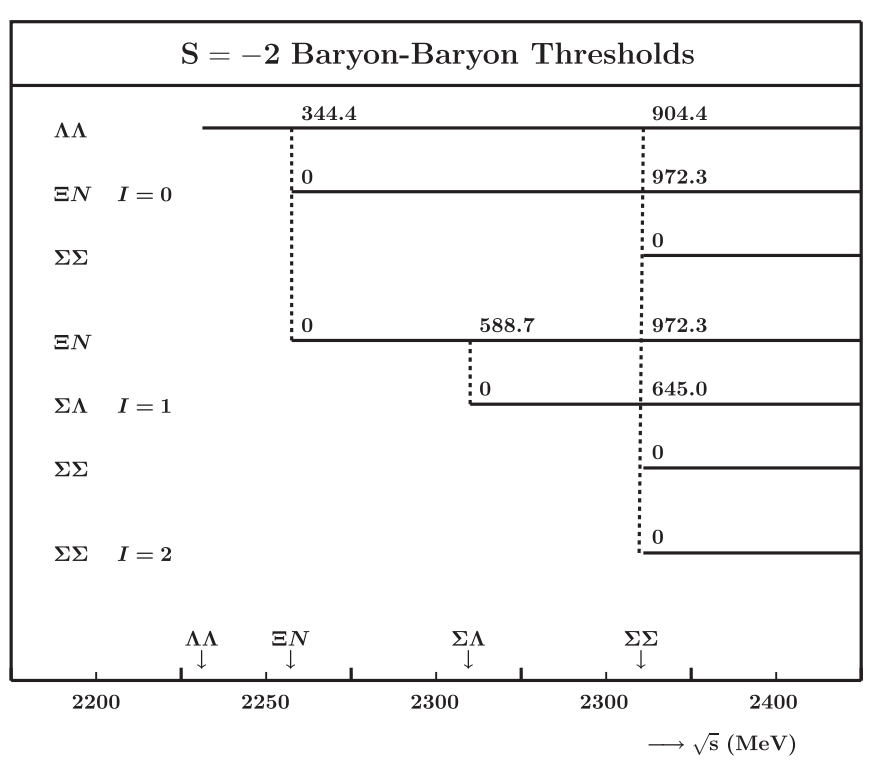

FIG. 2. Thresholds in $Y N$ and $Y Y$ channels for $S=-2 . \sqrt{s}$ is the total energy in the c.m.

\section{MULTICHANNEL THRESHOLDS AND POTENTIALS}

\section{A. Thresholds}

Clearly, the $S=-2$ two-baryon channels represent a number of separate coupled-channel systems, separated by the charge, see Eq. (2.2). A further subdivision is according to the total isospin. The different thresholds have been discussed in detail in Ref. [7], and we show these thresholds here in Fig. 2 for the purpose of general orientation. Their presence turns the Lippmann-Schwinger and Schrödinger equation into a coupled-channel matrix equation, where the different channels open up at different energies. In general one has a combination of 'open' and 'closed' channels. For a discussion of the solution of such a mixed system, we refer to Ref. [42].

\section{B. Threshold- and meson-mass corrections in potentials}

As discussed in Refs. [42,43], the one-meson-exchange Feynman graph consists actually of two three-dimensional time-ordered graphs. This has consequences for the range of the energy-independent meson exchange potentials.

This effect results in "effective" meson masses and obviously depends on the baryon masses. For more details on these effective meson masses for the $S=-2$ channels, we refer to Ref. [7]. The baryon masses used in this paper are about the same as in [7], and are given in Table V. In our potentials we have included the decrease in the physical pion mass of $138.041 \mathrm{MeV} / c^{2}$ to $132.58 \mathrm{MeV} / c^{2}$ in $\Lambda B \rightarrow \Sigma B$, where $B$ can be $N, \Sigma$, or $\Xi$, and the much more significant decrease to $114.62 \mathrm{MeV} / c^{2}$ in $\Sigma \Lambda \rightarrow \Lambda \Sigma$; in all other cases, we retain the physical value of $138.041 \mathrm{MeV} / c^{2}$. The $K$ and $K^{*}$ masses need to be reduced in all cases, where the drop in mass squared ranges from $\left(125.56 \mathrm{MeV} / c^{2}\right)^{2}$ in $\Xi \Sigma \rightarrow \Sigma \Xi$ to $\left(253.63 \mathrm{MeV} / c^{2}\right)^{2}$ in $\Sigma N \rightarrow N \Sigma$.

The used nonstrange meson masses are the same as in paper II [2], as well as the cut-off masses. The effective masses 
TABLE V. Baryon masses in $\mathrm{MeV} / c^{2}$.

\begin{tabular}{lcc}
\hline \hline Baryon & & Mass \\
\hline Nucleon & $p$ & 938.2796 \\
Hyperon & $n$ & 939.5731 \\
& $\Lambda$ & 1115.60 \\
& $\Sigma^{+}$ & 1189.37 \\
$\Sigma^{0}$ & 1192.46 \\
Cascade & $\Sigma^{-}$ & 1197.436 \\
& $\Xi^{0}$ & 1314.90 \\
& $\Xi^{-}$ & 1321.32 \\
\hline \hline
\end{tabular}

of the strangeness carrying mesons are $m_{K}=457.0, m_{K^{*}}=$ 872.464( $(I=0), m_{K^{*}}=872.890(I=1), m_{\kappa}=816.0$, and for the axial mesons $m_{K_{1 A}}=m_{K_{1 B}}=1321.0$.

\section{RESULTS}

The main purpose of this paper is to present the properties of the ESC16 potentials for the $S=-2$ sector. As described already, the free parameters in this model are fitted entirely to the $N N$ and $Y N$ scattering data for the $S=0$ and $S=-1$ sectors, respectively, with additional information from hypernuclei and hyperonic matter. Given the expressions for the coupling constants in terms of the octet, singlet couplings, and $F /(F+D)$ ratios and their values of papers I and II, it is straightforward to evaluate all possible baryon-baryon-meson coupling constants needed for the $S \leqslant-2$ potentials. A complete set of coupling constants for model ESC16 is given in Table VI.

In Figs. 3-7 we display the elastic channel $S$-wave potentials for the individual pseudoscalar (PS), vector (V), scalar (S), and axial (A) mesons in the case of model ESC16. Here, the mesons with strangeness $K, K^{*}, \kappa$, and $K_{1}$ do not
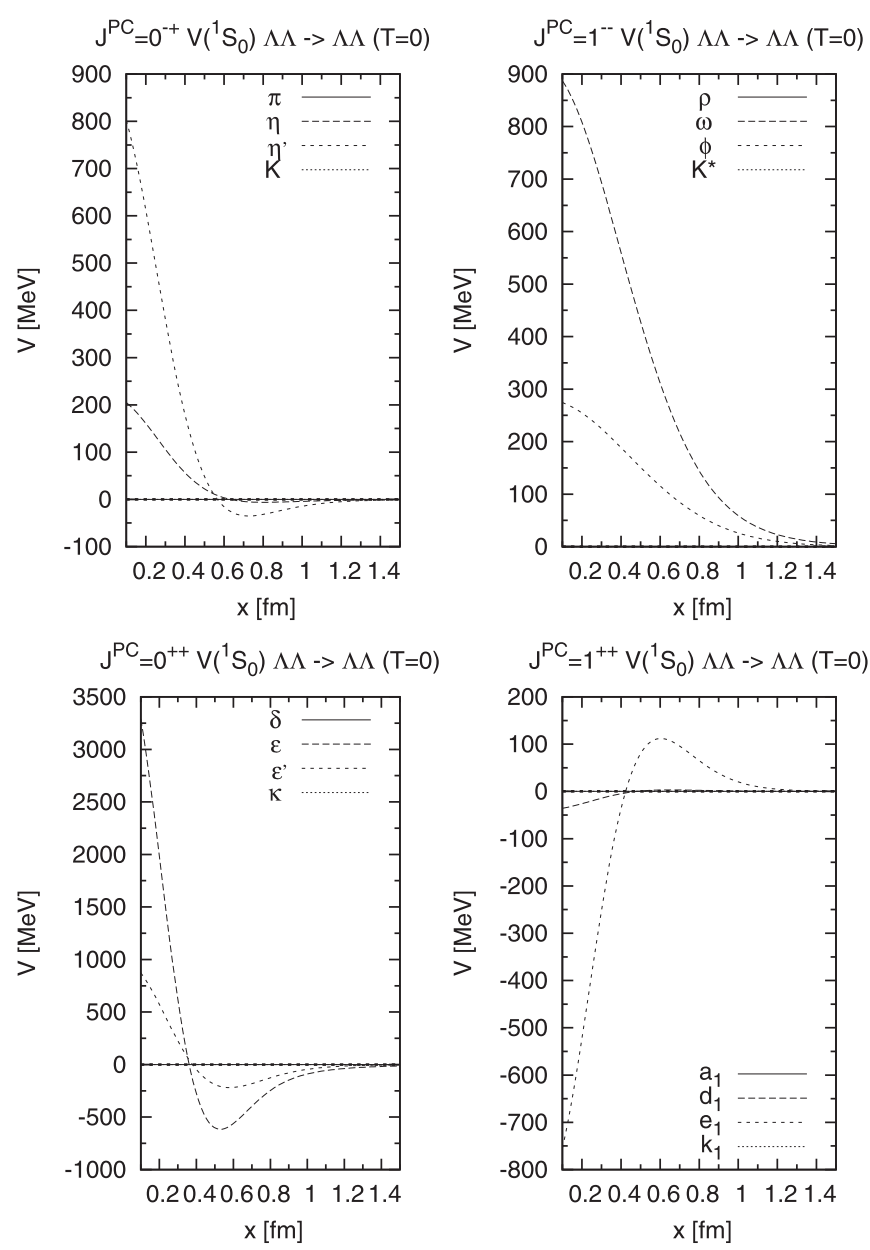

FIG. 3. ESC16: OBE contributions to the $\Lambda \Lambda\left({ }^{1} S_{0}, I=0\right)$ potentials for the PS, V, S, and $A$ meson nonets.

TABLE VI. Coupling constants for model ESC16, divided by $\sqrt{4 \pi} . M$ refers to the meson. The coupling constants are listed in the order pseudoscalar, vector ( $g$ and $f$ ), axial vector $A$ ( $g$ and $f$ ), scalar, axial vector $B$, and diffractive.

\begin{tabular}{|c|c|c|c|c|c|c|c|c|c|c|}
\hline$f$ & $\pi$ & 0.2684 & 0.1959 & 0.1968 & -0.0725 & $K$ & -0.2681 & 0.0713 & 0.0725 & -0.2684 \\
\hline$g$ & $\rho$ & 0.5793 & 1.1586 & 0.0000 & 0.5793 & $K^{*}$ & -1.0034 & 1.0034 & -0.5793 & -0.5793 \\
\hline$g$ & $a_{1}$ & -0.8172 & -0.6260 & -0.5822 & 0.1912 & $K_{1 A}$ & 0.8333 & -0.2511 & -0.1912 & 0.8172 \\
\hline$f$ & & -1.6521 & -1.2656 & -1.1770 & 0.3865 & & 1.6846 & -0.5076 & -0.3865 & 1.6521 \\
\hline$g$ & $a_{0}$ & 0.5393 & 1.0786 & 0.0000 & 0.5393 & $\kappa$ & -0.9341 & 0.9341 & -0.5393 & -0.5393 \\
\hline & $M$ & $N N M$ & $\Lambda \Lambda M$ & $\Sigma \Sigma M$ & $\Xi \Xi M$ & $M$ & $N N M$ & $\Lambda \Lambda M$ & $\Sigma \Sigma M$ & $\Xi \Xi M$ \\
\hline$f$ & $\eta$ & 0.1368 & -0.1259 & 0.2599 & -0.1958 & $\eta^{\prime}$ & 0.3181 & 0.3711 & 0.2933 & 0.3852 \\
\hline$g$ & $\omega$ & 3.1148 & 2.4820 & 2.4820 & 1.8492 & $\phi$ & -1.2384 & -2.0171 & -2.0171 & -2.7958 \\
\hline$f$ & & -0.5710 & -3.2282 & -0.2863 & -4.4144 & & 2.8878 & -0.3819 & 3.2380 & -1.8416 \\
\hline$g$ & $f_{1}^{\prime}$ & -0.7596 & -0.1213 & -1.0133 & 0.0710 & $f_{1}$ & 0.5147 & 1.0503 & 0.3019 & 1.2117 \\
\hline$g$ & $O$ & 4.1637 & 4.1637 & 4.1637 & 4.1637 & & & & & \\
\hline$f$ & & -3.8859 & -3.8859 & -3.8859 & -3.8859 & & & & & \\
\hline
\end{tabular}



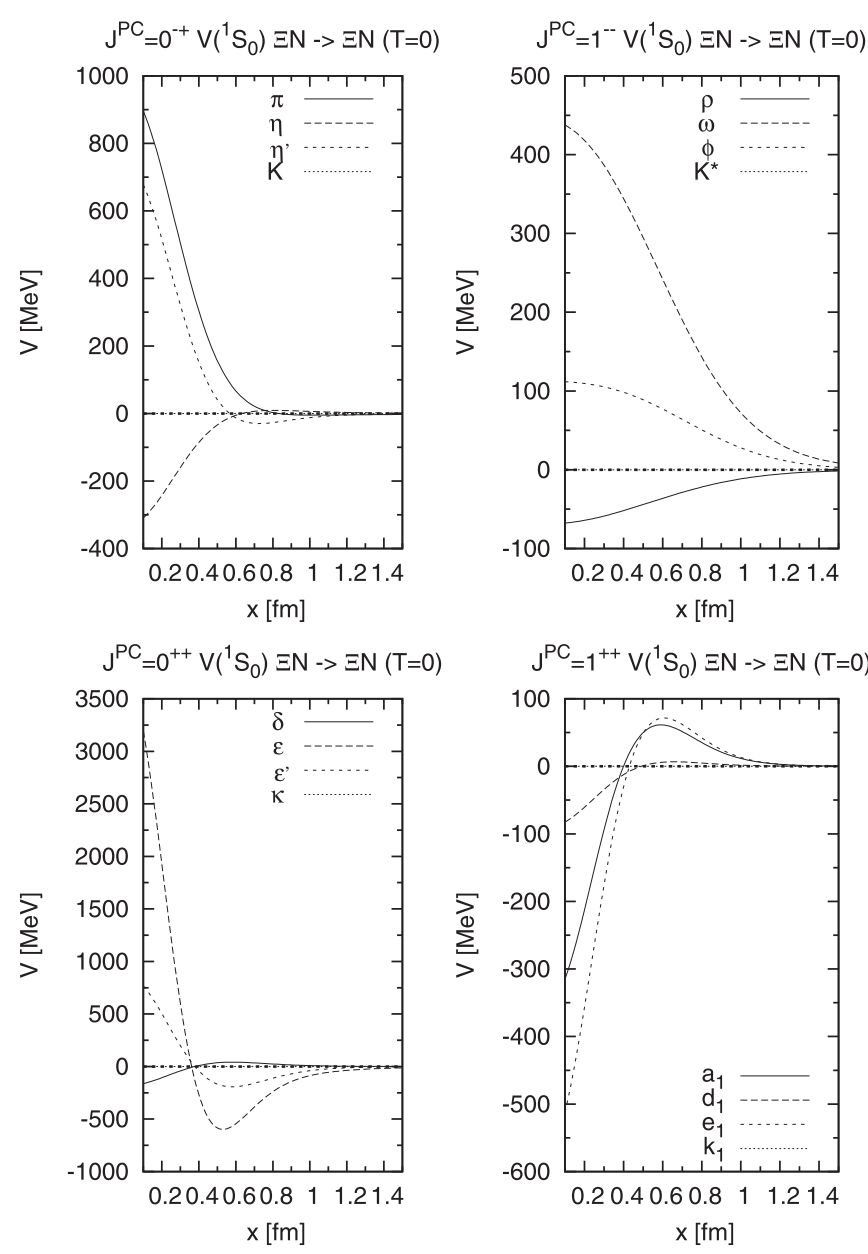

FIG. 4. ESC16: OBE contributions to the $\Xi N\left({ }^{1} S_{0}, I=0\right)$ potentials for the PS, V, S, and $A$ meson nonets.

contribute. In the captions of these figures and elsewhere $T$ denotes the total isospin $I$.

In Figs. 8-12 we display the global features of the $S$-wave elastic channel OBE, TPS, PAIR, and total potentials. In each case the total potentials show a large inner repulsion and a little or no attraction. In the $\Lambda \Lambda$ case the medium range attraction is due to the TPS contribution. The TPS potentials give also attraction in the other channels. The OBE potentials give repulsion in all these channels, and the PAIR potentials give a short range attraction in the $\Lambda \Lambda\left({ }^{1} S_{0}\right)$ and $\Xi N\left({ }^{1} S_{0}, I=0\right)$ channel.

In the following we will present the model predictions for scattering lengths, bound states, and cross sections.

\section{A. Effective-range parameters}

For ESC16 the $I=0$ low-energy parameters are

$$
\begin{array}{ll}
a_{\Lambda \Lambda}\left({ }^{1} S_{0}\right)=-0.439[\mathrm{fm}], & r_{\Lambda \Lambda}\left({ }^{1} S_{0}\right)=9.533[\mathrm{fm}] . \\
a_{\Xi N}\left({ }^{3} S_{1}\right)=-0.269[\mathrm{fm}], & r_{\Xi N}\left({ }^{3} S_{1}\right)=-10.250[\mathrm{fm}] .
\end{array}
$$

For $I=1$ we have for ESC16

$$
a_{\Xi N}\left({ }^{1} S_{0}\right)=0.556[\mathrm{fm}], \quad r_{\Xi N}\left({ }^{1} S_{0}\right)=-3.043[\mathrm{fm}],
$$
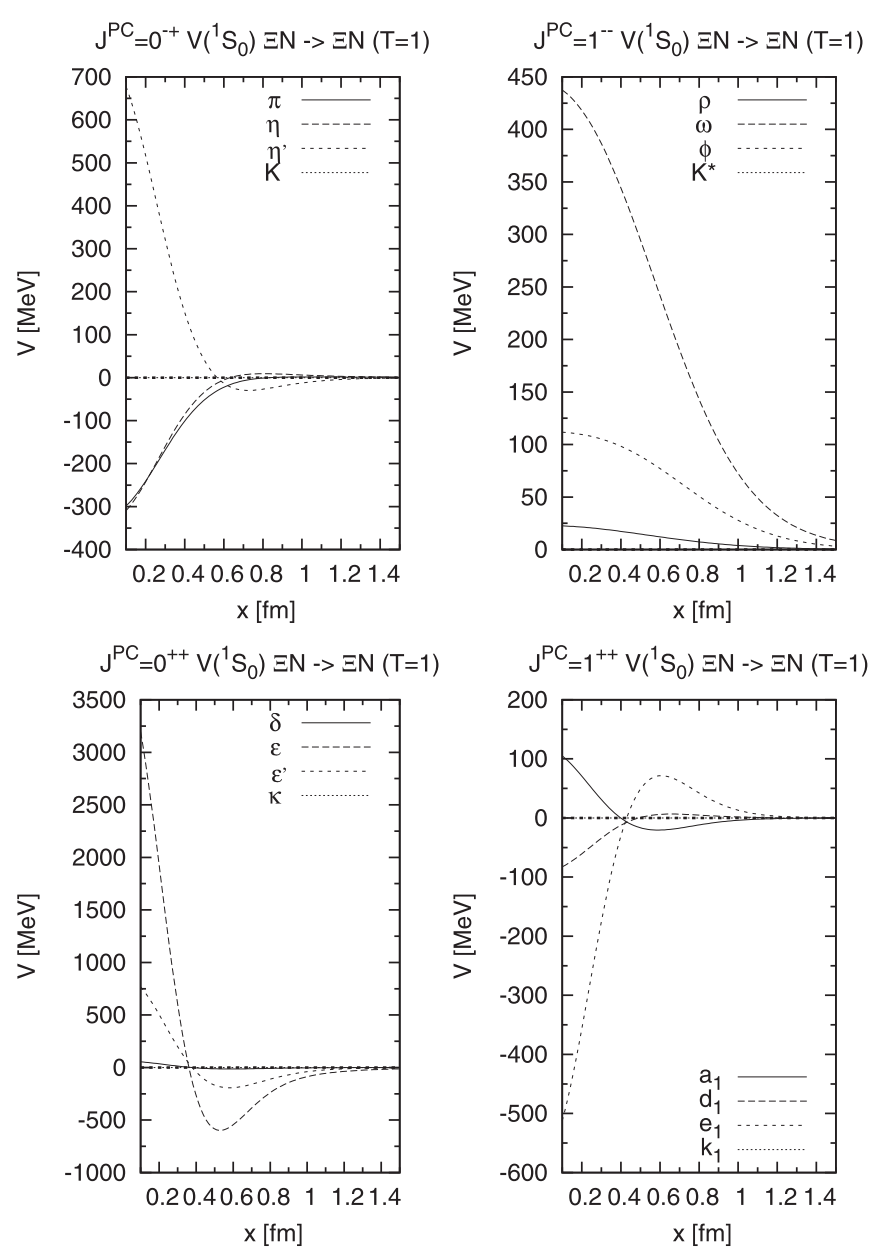

FIG. 5. $\Xi N\left({ }^{1} S_{0}, I=1\right)$ potentials.

$$
a_{\Xi N}\left({ }^{3} S_{1}\right)=0.144[\mathrm{fm}], \quad r_{\Xi N}\left({ }^{3} S_{1}\right)=41.005[\mathrm{fm}],
$$

and for $\Sigma \Sigma\left({ }^{1} S_{0}, I=2\right)$ we have for ESC16

$$
\begin{array}{rr}
a_{\Sigma^{0} \Sigma^{0}}=+0.495[\mathrm{fm}], & r_{\Sigma^{0} \Sigma^{0}}=+11.943[\mathrm{fm}], \\
a_{\Sigma^{ \pm} \Sigma^{ \pm}}=-0.432[\mathrm{fm}], & r_{\Sigma^{ \pm} \Sigma^{ \pm}}=-594.51[\mathrm{fm}], \\
a_{\Sigma^{ \pm} \Sigma^{\mp}}=+9.983[\mathrm{fm}], & r_{\Sigma^{ \pm} \Sigma^{\mp}}=-44.578[\mathrm{fm}] .
\end{array}
$$

We note that the $\Sigma \Sigma(I=2)$ channels are purely $\{27\}$ irrep, like the $p p$ and $\Sigma^{+} p\left({ }^{1} S_{0}, I=3 / 2\right)$. This implies that the nuclear interaction is rather attractive and rather close to a bound-state. In contrast to $p p$ and $\Sigma^{+} p$ in the channels $\Sigma^{ \pm} \Sigma^{\mp}$ the Coulomb is attractive.

The results at the $\Xi N$ threshold and at the $\Lambda \Sigma$ threshold are given in Tables VII and VIII. The $\Lambda \Lambda\left({ }^{1} S_{0}\right)$ scattering lengths are found to be larger in absolute value than in the NSC97 models [7], indicating a more attractive $\Lambda \Lambda$ interaction.

The old experimental information ${ }_{\Lambda \Lambda}^{6} \mathrm{He}$ seemed to indicate a separation energy of $\Delta B_{\Lambda \Lambda}=4-5 \mathrm{MeV}$, corresponding to a rather strong attractive $\Lambda \Lambda$ interaction. As a matter of fact, an estimate for the $\Lambda \Lambda^{1} S_{0}$ scattering length, based on such a value for $\Delta B_{\Lambda \Lambda}$, gives $a_{\Lambda \Lambda}\left({ }^{1} S_{0}\right) \approx-2.0 \mathrm{fm}[44,45]$. However, in recent years the experimental information and interpretation of the ground state levels of ${ }_{\Lambda \Lambda}^{6} \mathrm{He},{ }_{\Lambda \Lambda}^{10} \mathrm{Be}$, and ${ }_{\Lambda \Lambda}^{13} \mathrm{~B}$ [46], has been changed drastically. This because of the 

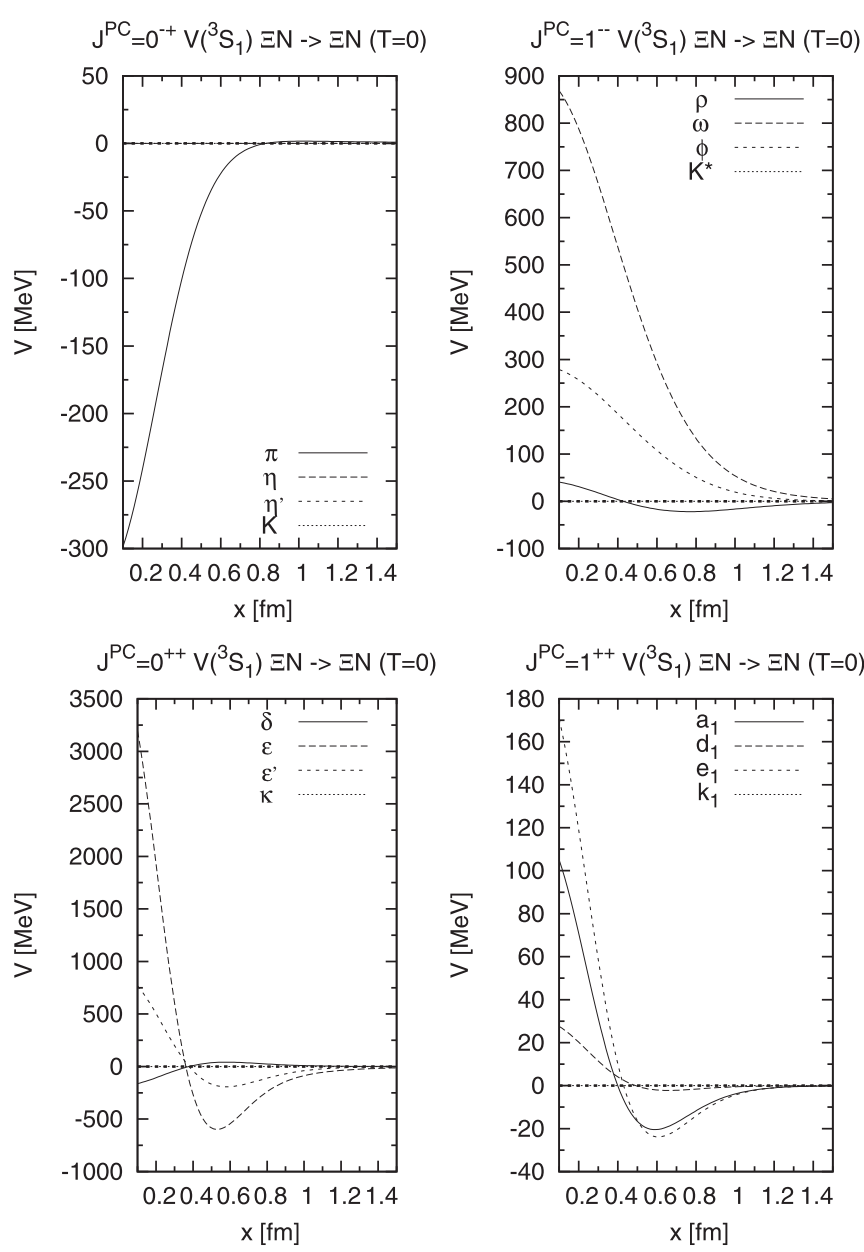

FIG. 6. $\Xi N\left({ }^{3} S_{1}, I=0\right)$ potentials.

Nagara event, identified uniquely as ${ }_{\Lambda \Lambda}^{6} \mathrm{He}$ [8], which established that the $\Lambda \Lambda$ interaction is weaker $\left(\Delta B_{\Lambda \Lambda} \approx 0.7 \mathrm{MeV}\right)$. Furthermore, in $\Lambda \Lambda$ correlation studies of heavy-ion collisions [47] a small negative $a_{\Lambda \Lambda}\left({ }^{1} S_{0}\right)$ is favored.

In NSC97 [23] it was only possible to increase the attraction in the $\Lambda \Lambda$ channel by modifying the scalar-exchange potential. If the scalar mesons are viewed as being mainly $q \bar{q}$ states, one finds that the (attractive) scalar-exchange part of

TABLE VII. ESC16: Inverse-scattering-length and effectiverange matrices at (i) the $\Xi N$ threshold for $I=0$, and (ii) the $\Lambda \Sigma$ threshold for $I=1$. The order of the states (1-2) reads $\Lambda \Lambda\left({ }^{1} S_{0}\right), \Xi N\left({ }^{1} S_{0}\right)$, and $\Xi N\left({ }^{1} S_{0}\right), \Lambda \Sigma\left({ }^{1} S_{0}\right)$ for, respectively, $I=0$ and $I=1$. The dimensions of the matrix elements are in $[\mathrm{fm}]^{-1}\left(A^{-1}\right)$ and $[\mathrm{fm}](R)$.

\begin{tabular}{|c|c|c|c|c|}
\hline & \multicolumn{2}{|c|}{$\Xi N$ threshold } & \multicolumn{2}{|c|}{$\Lambda \Sigma$ threshold } \\
\hline & $A^{-1}$ & $R$ & $A^{-1}$ & $R$ \\
\hline 11 & 0.234 & 13.557 & -0.085 & 8.987 \\
\hline 12 & 1.607 & -0.377 & -1.423 & 6.458 \\
\hline 22 & 0.569 & 2.610 & -0.485 & 6.012 \\
\hline
\end{tabular}
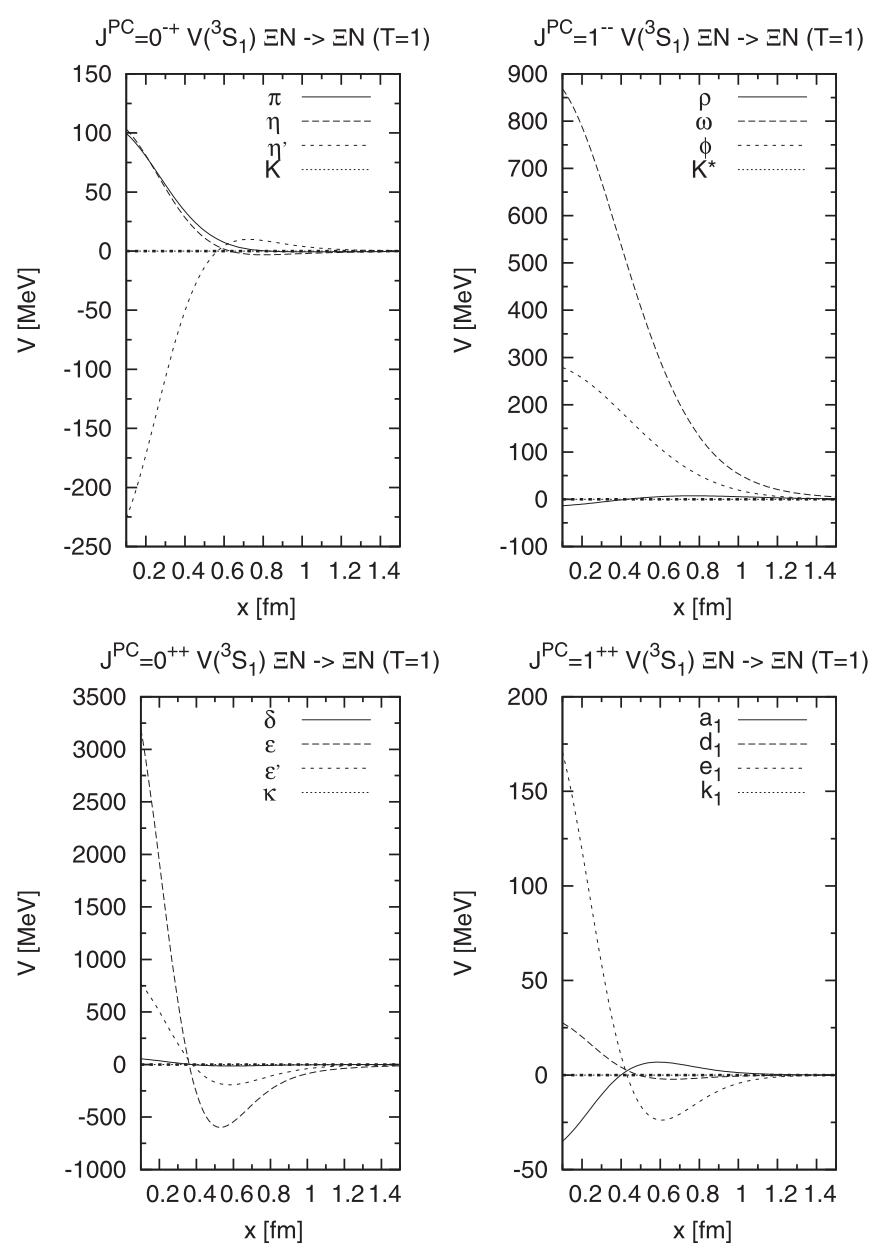

FIG. 7. $\Xi N\left({ }^{3} S_{1}, I=1\right)$ potentials.

the interaction in the various channels satisfies

$$
\left|V_{\Lambda \Lambda}\right|<\left|V_{\Lambda N}\right|<\left|V_{N N}\right|
$$

suggesting indeed a rather weak $\Lambda \Lambda$ potential. The NSC97 fits to the $Y N$ scattering data [23] give values for the scalar-meson mixing angle which seem to point to almost ideal mixing for the scalars as $q \bar{q}$ states. We found that an increased attraction in the $\Lambda \Lambda$ channel would give rise to (experimentally unobserved) deeply bound states in the $\Lambda N$

TABLE VIII. $I=1$ : Inverse-scattering-length and effectiverange matrices at the $\Lambda \Sigma$ threshold. The order of the states (1-2) reads $\Xi N\left({ }^{3} S_{1}\right), \Xi N\left({ }^{3} D_{1}\right), \Lambda \Sigma\left({ }^{3} S_{1}\right)$. The dimension of the matrix elements are in $[\mathrm{fm}]^{-1-l-l^{\prime}}\left(A^{-1}\right)$ and $[\mathrm{fm}]^{1-l-l^{\prime}}(R)$.

\begin{tabular}{lrr}
\hline \hline & \multicolumn{2}{c}{ ESC16 } \\
\cline { 2 - 3 } & $A^{-1}$ & $R$ \\
\hline 11 & 4.637 & 37.197 \\
12 & -21.474 & -94.878 \\
13 & 2.050 & 18.181 \\
22 & 115.960 & 506.653 \\
23 & 5.839 & -88.231 \\
33 & 0.954 & 8.645 \\
\hline \hline
\end{tabular}



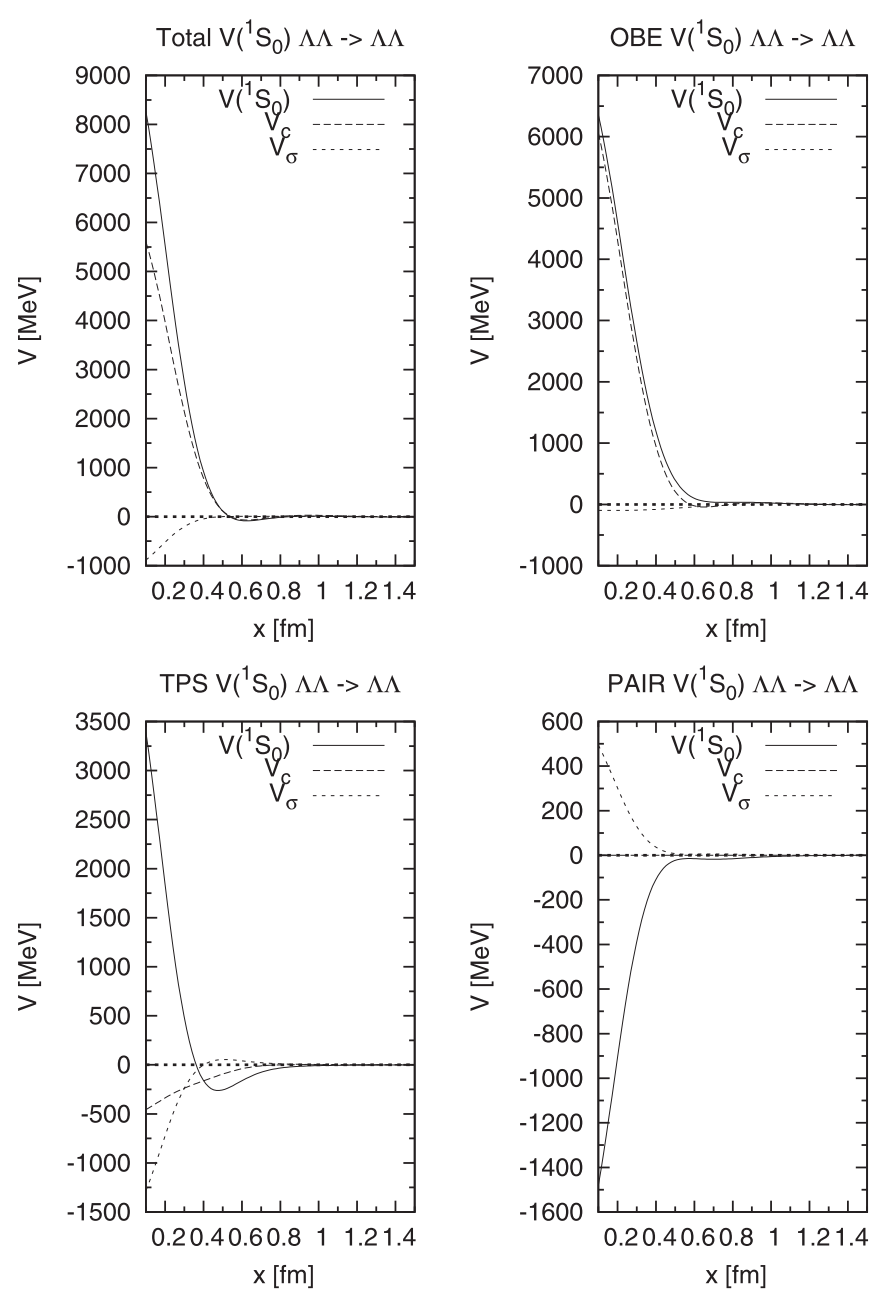

FIG. 8. $\Lambda \Lambda\left({ }^{1} S_{0}, I=0\right)$ potentials.

channel. On the other hand, in the ESC models there are in principle more possibilities because of the presence of mesonpair potentials. As one sees from the values of the $a_{\Lambda \Lambda}\left({ }^{1} S_{0}\right)$ in the ESC16 model of this paper, we can produce the apparently required attraction in the $\Lambda \Lambda$ interaction without giving rise to $\Lambda N$ bound states. Notice that also in ESC08 we have almost ideal scalar mixings, akin to NSC97.

\section{B. No deuteron state in $\Xi N\left({ }^{3} S_{1}-{ }^{3} D_{1}, I=1\right)$}

A discussion of the possible bound states, using the $\mathrm{SU}(3)$ content of the different $S=0,-1,-2$ channels is given in Ref. [7]. As in Ref. [7], for a general orientation, we list in Table IX all the irreps to which the various baryon-baryon channels belong.

In ESC16 there is no bound-state with isospin $I=1$ or $I=0$ and strangeness $S=-2$. Apparently, the tensor contribution in the spin triplet-coupled states is not large enough to give a bound state. (The $\Xi N \rightarrow Y Y$ tensor potentials for isospin $I=T=1$ are exhibited in Fig. 13.) In ESC08c a "phenomenological" deuteron-like $D^{\star}$ was produced belonging to the $\left\{10^{*}\right\} \mathrm{SU}(3)$ irrep, which is a $\Xi N$ bound state in the ${ }^{3} S_{1}{ }^{3} D_{1}$ coupled partial wave. In model ESC04d [5], there occurs a $\Xi N$ bound state in the $\Xi N\left({ }^{3} S_{1}-{ }^{3} D_{1},(I=0)\right.$ partial
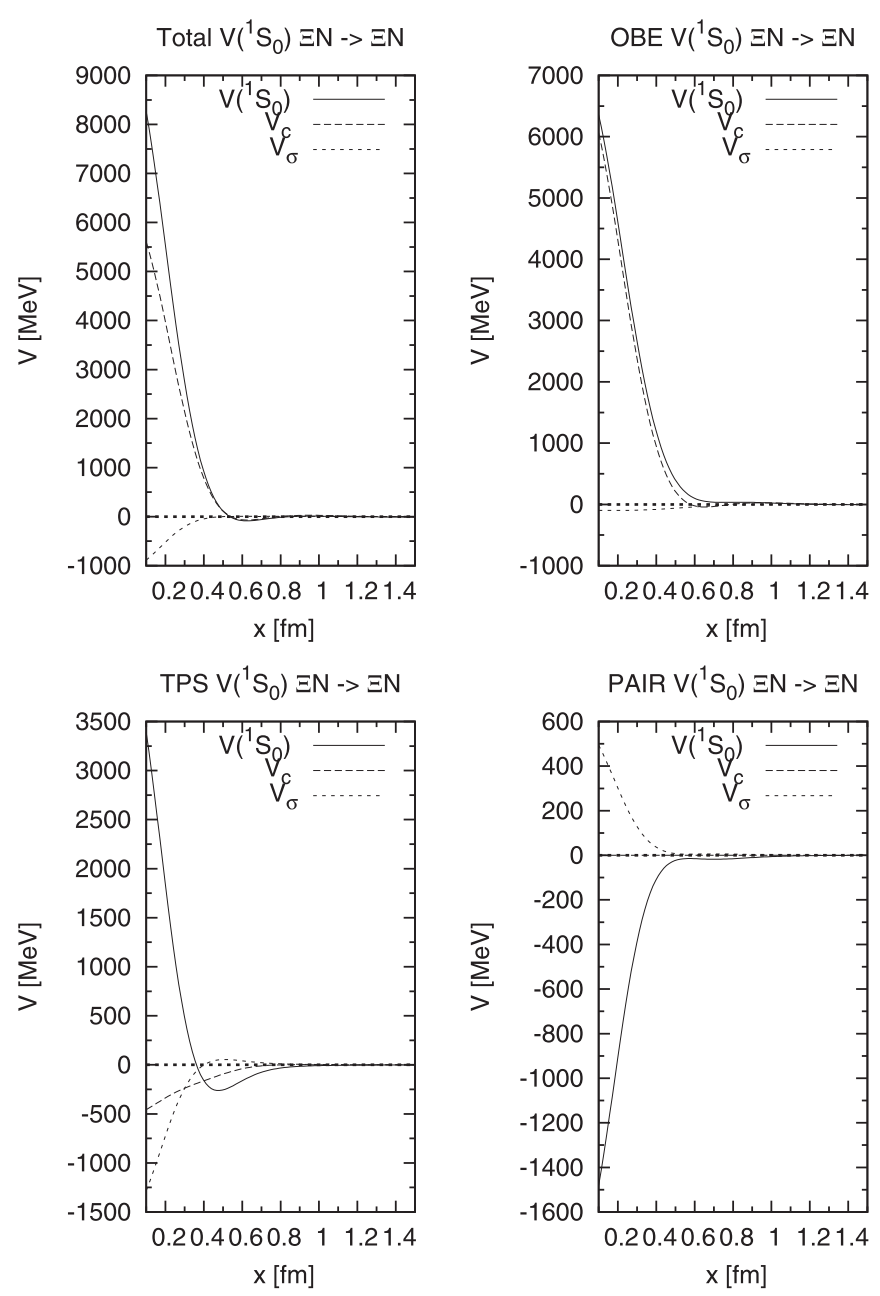

FIG. 9. $\Xi N\left({ }^{1} S_{0}, I=0\right)$ potentials.

TABLE IX. SU(3) content of the different interaction channels. $S$ is the total strangeness and $I$ is the isospin. The upper half refers to the space-spin symmetric states ${ }^{3} S_{1},{ }^{1} P_{1},{ }^{3} D, \ldots$, while the lower half refers to the space-spin antisymmetric states ${ }^{1} S_{0},{ }^{3} P,{ }^{1} D_{2}, \ldots$.

\begin{tabular}{|c|c|c|c|}
\hline \multirow[b]{2}{*}{$S$} & \multicolumn{3}{|c|}{ Space-spin symmetric } \\
\hline & $I$ & Channels & SU(3) irreps \\
\hline 0 & 0 & $N N$ & $\left\{10^{*}\right\}$ \\
\hline \multirow[t]{2}{*}{-1} & $1 / 2$ & $\Lambda N, \Sigma N$ & $\left\{10^{*}\right\},\{8\}_{a}$ \\
\hline & $3 / 2$ & $\Sigma N$ & $\{10\}$ \\
\hline \multirow{4}{*}{-2} & 0 & $\Xi N$ & $\{8\}_{a}$ \\
\hline & 1 & $\Xi N, \Sigma \Sigma$ & $\{10\},\left\{10^{*}\right\},\{8\}_{a}$ \\
\hline & & $\Sigma \Lambda$ & $\{10\},\left\{10^{*}\right\}$ \\
\hline & \multicolumn{3}{|c|}{ Space-spin antisymmetric } \\
\hline$S$ & $I$ & Channels & SU(3) irreps \\
\hline 0 & 1 & $N N$ & $\{27\}$ \\
\hline \multirow[t]{2}{*}{-1} & $1 / 2$ & $\Lambda N, \Sigma N$ & $\{27\},\{8\}_{s}$ \\
\hline & $3 / 2$ & $\Sigma N$ & $\{27\}$ \\
\hline \multirow[t]{3}{*}{-2} & 0 & $\Lambda \Lambda, \Xi N, \Sigma \Sigma$ & $\{27\},\{8\}_{s},\{1\}$ \\
\hline & 1 & $\Xi N, \Sigma \Lambda$ & $\{27\},\{8\}_{s}$ \\
\hline & 2 & $\Sigma \Sigma$ & $\{27\}$ \\
\hline
\end{tabular}



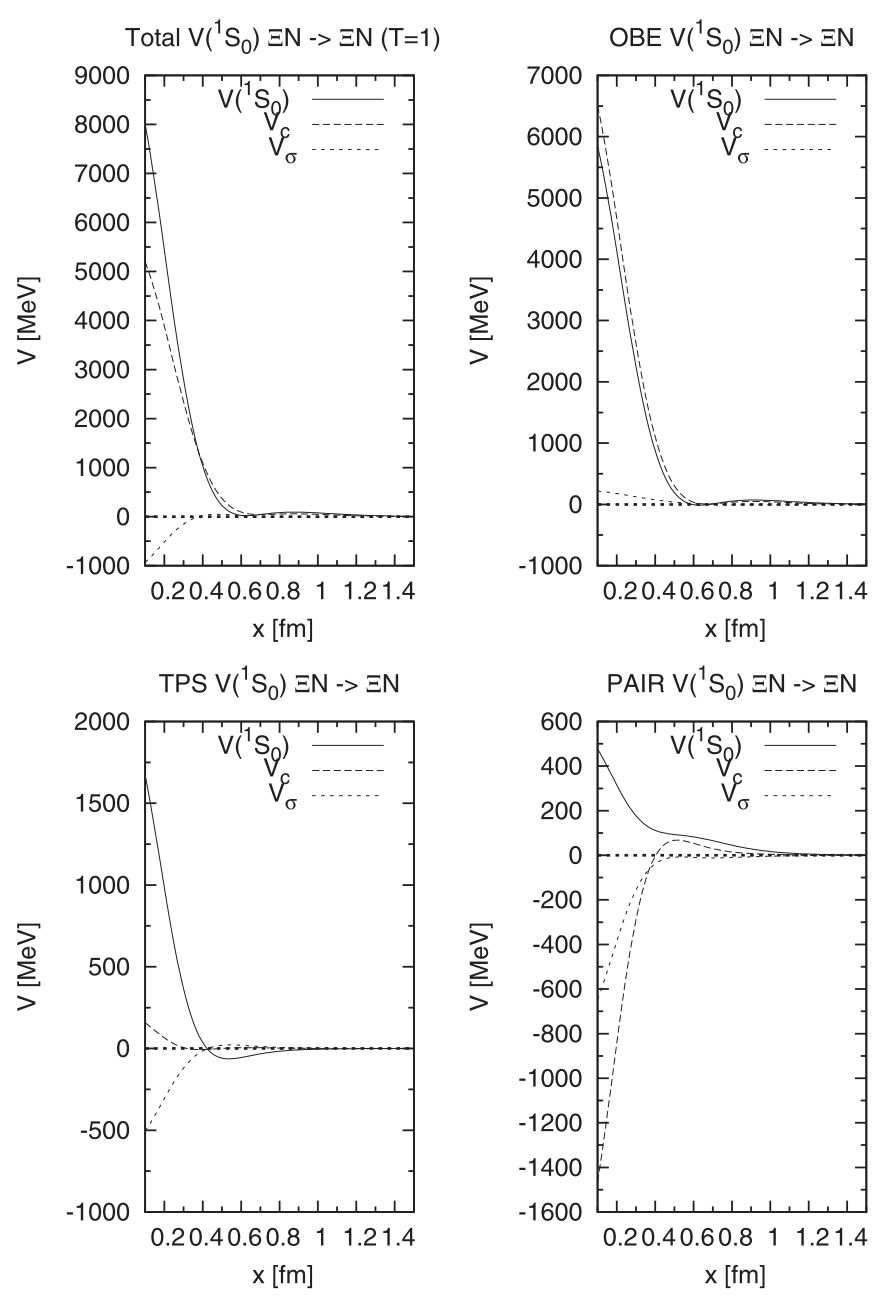

FIG. 10. $\Xi N\left({ }^{1} S_{0}, I=1\right)$ potentials.

wave. From Table IX one sees that this is a $\left\{8_{a}\right\}$ state, which was a little bit surprising, because the OBE-potential one expects to be rather repulsive in the irrep $\left\{8_{a}\right\}$, see Ref. [24]. In the ESC04 models this occurrence was ascribed to the inclusion of the potentials of the axial-vector mesons, and the meson pairs. Since the ESC04a-c versions did not show such a bound state it is considered to be accidental.

\section{Partial wave phase parameters}

For the BB channels below the inelastic threshold we use for the parametrization of the amplitudes the standard nuclear-bar phase shifts [48]. The information on the elastic amplitudes above thresholds is most conveniently given using the BKS phases [25-27]. For uncoupled partial waves, the elastic BB $S$-matrix element is parametrized as

$$
S=\eta e^{2 i \delta}, \quad \eta=\cos (2 \rho) .
$$

For coupled partial waves the elastic BB amplitudes are $2 \times 2$ matrices. The BKS $S$-matrix parametrization, which is of the type- $S$ variety, is given by

$$
S=e^{i \delta} e^{i \epsilon} N e^{i \epsilon} e^{i \delta}
$$
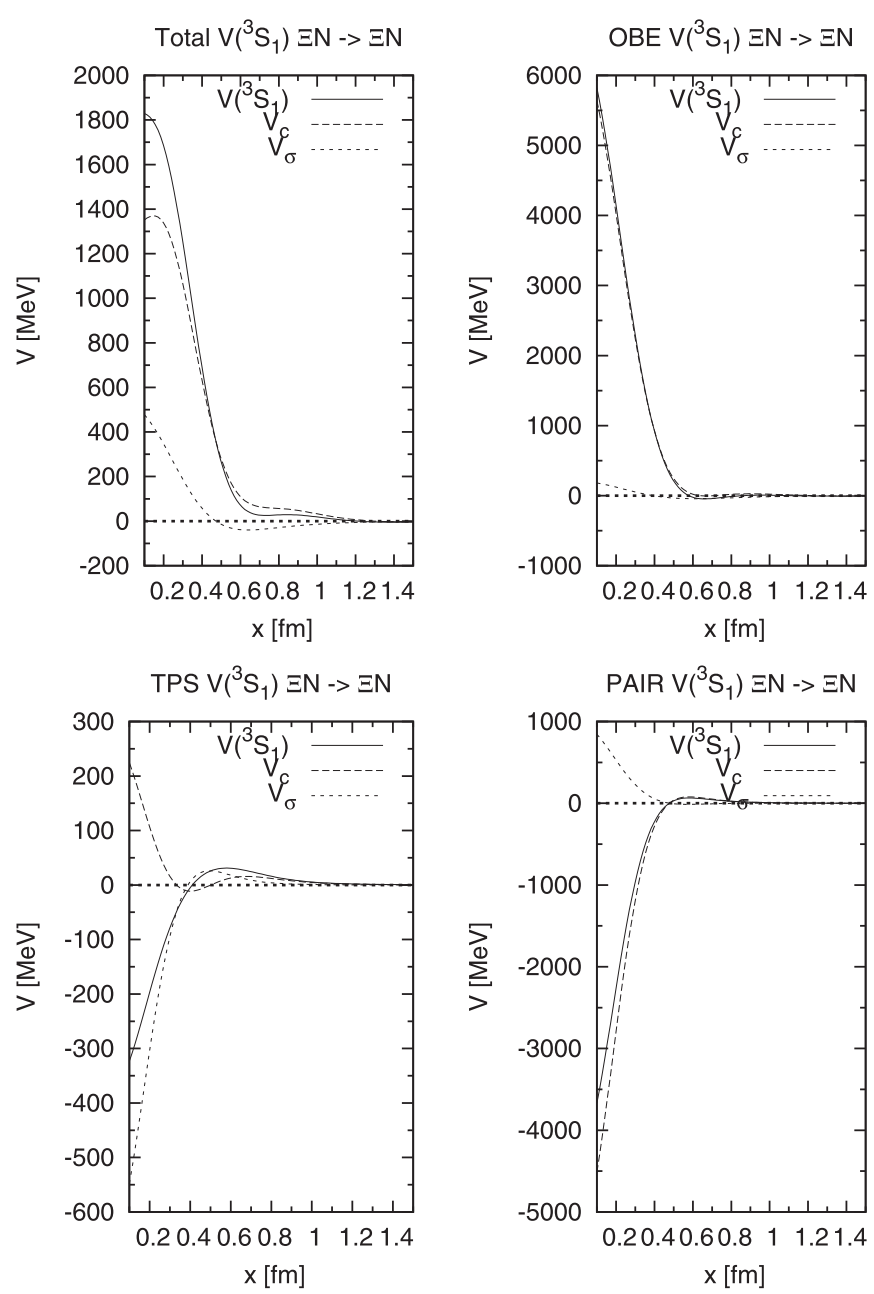

FIG. 11. $\Xi N\left({ }^{3} S_{1}, I=0\right)$ potentials.

where

$$
\delta=\left(\begin{array}{cc}
\delta_{\alpha} & 0 \\
0 & \delta_{\beta}
\end{array}\right), \quad \epsilon=\left(\begin{array}{ll}
0 & \epsilon \\
\epsilon & 0
\end{array}\right),
$$

and $N$ is a real, symmetric matrix parametrized as

$$
N=\left(\begin{array}{ll}
\eta_{11} & \eta_{12} \\
\eta_{12} & \eta_{22}
\end{array}\right)
$$

From the various parametrizations of the $N$ matrix, we choose the Kabir-Kermode parametrization [28] to represent the $N$ matrix in the figures. Then, the $N$ matrix is given by the inelasticity parameters $(\alpha, \beta, \varphi)$, called $\rho$ parameters, as follows:

$$
N=\left(\begin{array}{cc}
\cos (2 \alpha) & \sin (\varphi+\xi) \\
\sin (\varphi+\xi) & \cos (2 \beta)
\end{array}\right)
$$

where

$$
\begin{aligned}
& \alpha= \pm \frac{1}{2} \cos ^{-1}\left(\eta_{11}\right), \quad \beta= \pm \frac{1}{2} \cos ^{-1}\left(\eta_{22}\right), \\
& \varphi=\sin ^{-1}\left(\eta_{12}\right)-\operatorname{sgn}\left(\eta_{12}\right) \sin ^{-1} Q, \\
& \xi=\operatorname{sgn}\left(\eta_{12}\right) \sin ^{-1} Q .
\end{aligned}
$$

Here,

$$
Q^{2}=1-\left|\eta_{11}+\eta_{22}\right|+\eta_{11} \eta_{22}
$$



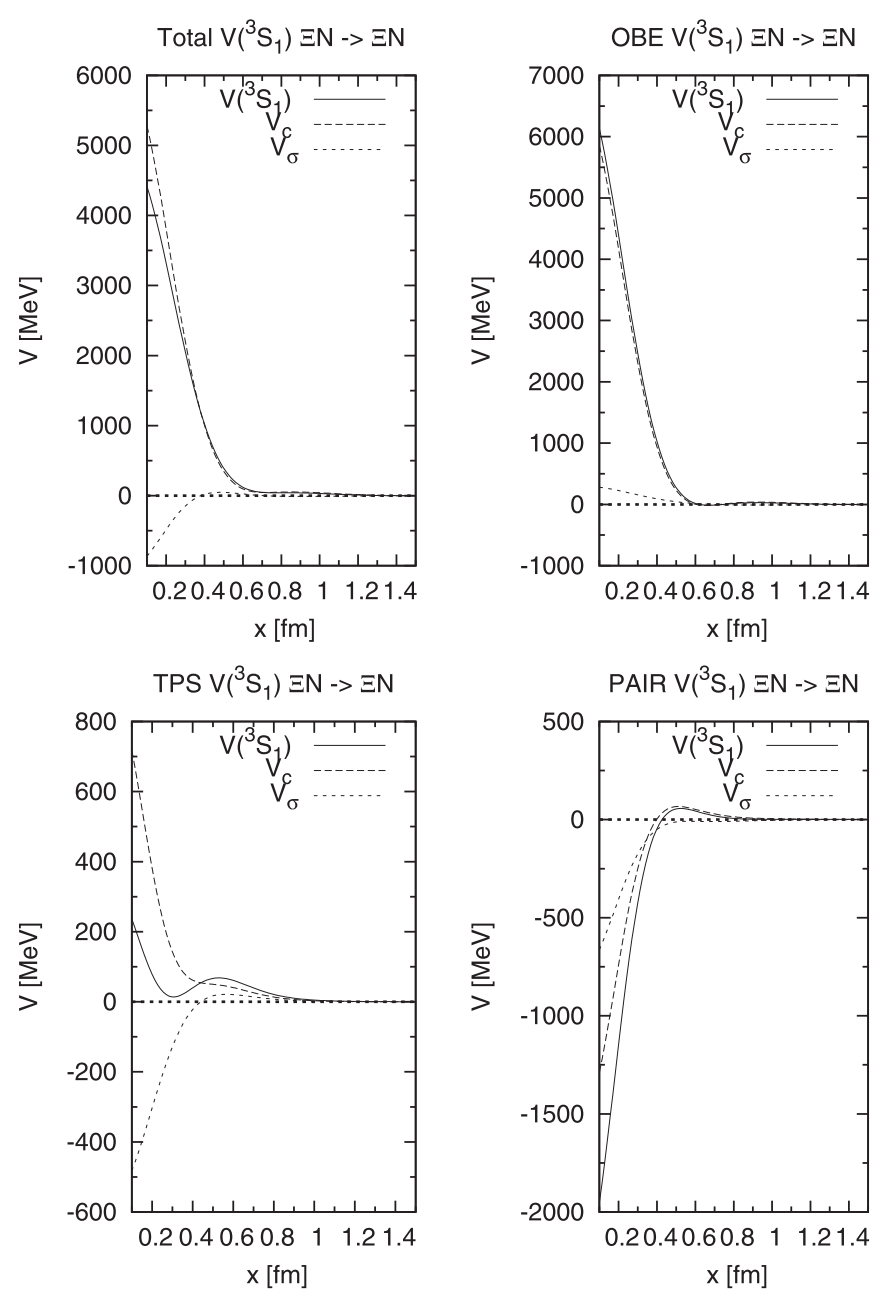

FIG. 12. $\Xi N\left({ }^{3} S_{1}, I=1\right)$ potentials.

In Tables XXIX-XXXV, we give for ESC16 the phases and inelasticity parameters $\rho$ and $\eta_{11}, \eta_{12}, \eta_{22}$, which enable the reader to construct the $N$ matrix most directly.

\section{Total cross sections}

We next present the predictions for the total cross section for several channels. We suppose always that the beam as well as the target are unpolarized. Therefore, we included the statistical factors, which are 1/4 for the spin-singlet and 3/4 for the spin-triplet case.

For those cases where both baryons are charged, we do not include the purely Coulomb contribution to the total cross section, nor do we include the Coulomb interference to the nuclear amplitude. The cross section is calculated by summing the contributions from partial waves with orbital angular momentum up to and including $L=2$. We find this to be sufficient for all the $S \neq 0$ sectors; inclusion of any higher partial waves has no significant effect. Inclusion of higher partial waves will shift the total cross section to slightly higher values without changing the overall shape. Of course, their inclusion would be necessary if a detailed comparison with real accurate experimental data were to be made.
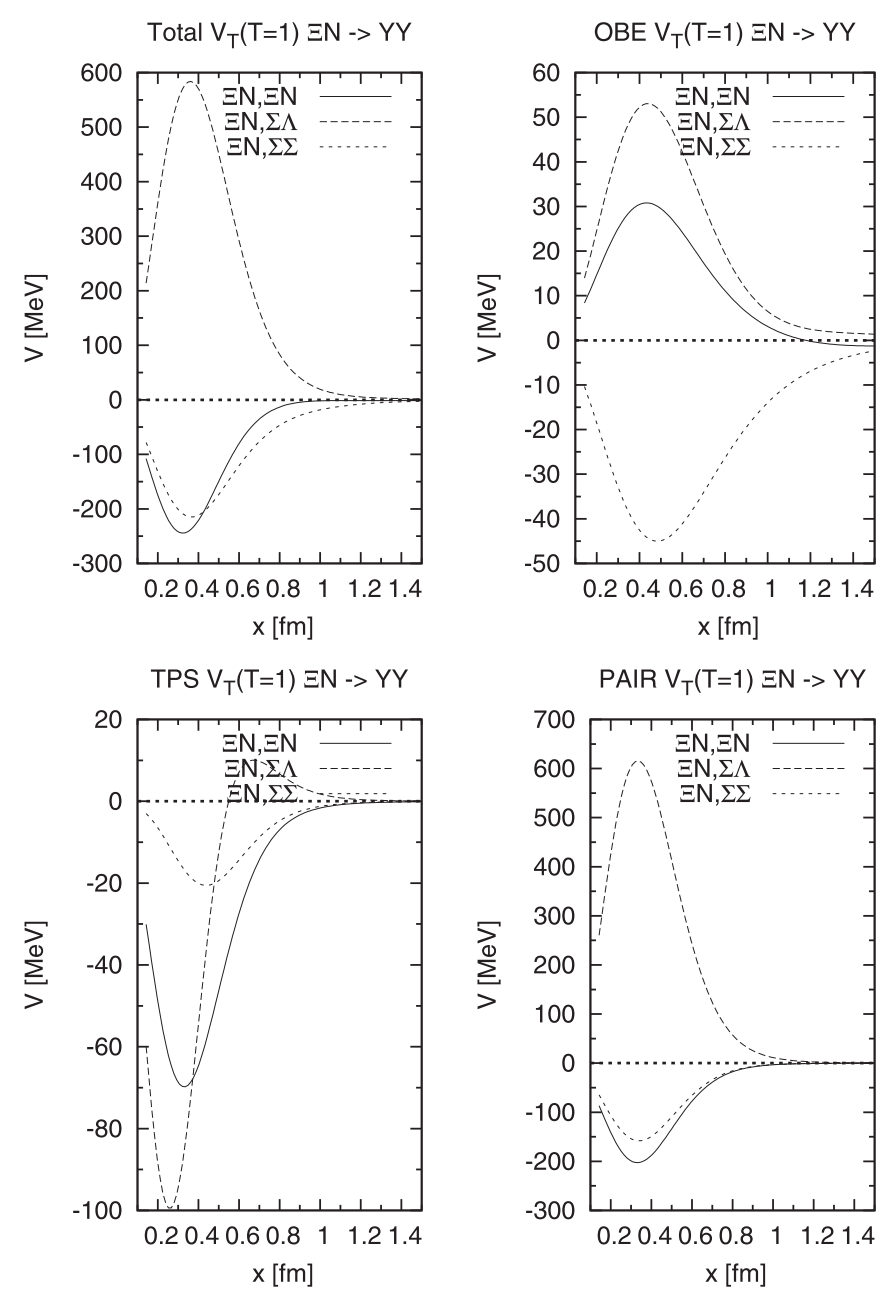

FIG. 13. $\Xi N\left({ }^{3} S_{1}, T=1\right)$ tensor potentials.

TABLE X. ESC16 $(I=0, L \leqslant 2)$ total cross sections in $(\mathrm{mb})$ as a function of the beam particle laboratory momentum $p_{\Lambda}$ in $(\mathrm{MeV} / c)$.

\begin{tabular}{lccccr}
\hline \hline & \multicolumn{2}{c}{$\Lambda \Lambda \rightarrow \Lambda \Lambda, \Xi N$} & & \multicolumn{2}{c}{$\Xi N \rightarrow \Xi N, \Lambda \Lambda$} \\
\cline { 2 - 3 }$p_{\text {Lab }}$ & $\Lambda \Lambda$ & $\Xi N$ & & $\Xi N$ & $\Lambda \Lambda$ \\
\hline 10 & 3.38 & - & 450.94 & 8.21 \\
50 & 3.14 & - & 83.62 & 8.16 \\
100 & 2.51 & - & 37.06 & 8.93 \\
150 & 1.77 & - & 21.83 & 10.69 \\
200 & 1.12 & - & 14.63 & 12.66 \\
250 & 0.66 & - & 10.63 & 14.33 \\
300 & 0.41 & - & 8.19 & 15.67 \\
350 & 0.64 & 1.65 & 6.60 & 16.80 \\
400 & 0.38 & 2.84 & 5.52 & 17.82 \\
500 & 0.73 & 2.59 & 4.21 & 19.74 \\
600 & 1.43 & 2.35 & 3.50 & 21.44 \\
700 & 2.25 & 2.24 & 3.11 & 22.90 \\
800 & 3.09 & 2.30 & 2.96 & 24.17 \\
900 & 3.92 & 2.61 & 3.02 & 25.32 \\
1000 & 4.84 & 2.92 & 3.36 & 26.40 \\
\hline \hline
\end{tabular}


TABLE XI. ESC16 $(I=1, L \leqslant 2)$ total cross sections $\Xi N \rightarrow$ $\Xi N, \Sigma \Lambda$ in $(\mathrm{mb})$ as a function of the beam particle laboratory beam particle momentum $p_{\text {Lab }}$ in $(\mathrm{MeV} / c)$.

\begin{tabular}{|c|c|c|c|c|c|}
\hline \multirow[b]{2}{*}{$p_{\text {lab }}$} & \multicolumn{2}{|c|}{$\Xi N \rightarrow \Xi N, \Sigma \Lambda$} & \multicolumn{3}{|c|}{$\Sigma \Lambda \rightarrow \Sigma \Lambda, \Xi N, \Sigma \Sigma$} \\
\hline & $\Xi N$ & $\Sigma \Lambda$ & $\Sigma \Lambda$ & $\Xi N$ & $\Sigma \Sigma$ \\
\hline 10 & 14.12 & - & 697.14 & 458.50 & - \\
\hline 50 & 13.05 & - & 215.80 & 11.93 & - \\
\hline 100 & 12.95 & - & 94.94 & 8.23 & - \\
\hline 200 & 13.22 & - & 38.34 & 6.39 & - \\
\hline 300 & 13.78 & - & 22.85 & 7.50 & - \\
\hline 400 & 14.07 & - & 16.53 & 9.45 & - \\
\hline 500 & 13.74 & - & 13.25 & 11.36 & - \\
\hline 600 & 10.13 & 3.62 & 11.35 & 12.91 & - \\
\hline 650 & 12.05 & 4.64 & 10.94 & 14.25 & 2.74 \\
\hline 700 & 13.28 & 6.18 & 9.52 & 14.80 & 6.26 \\
\hline 800 & 15.01 & 6.58 & 7.85 & 16.08 & 7.99 \\
\hline 900 & 16.37 & 6.65 & 6.64 & 17.04 & 7.81 \\
\hline 950 & 17.06 & 6.74 & 6.15 & 17.43 & 7.47 \\
\hline 1000 & 17.67 & 6.49 & 5.71 & 17.77 & 7.06 \\
\hline
\end{tabular}

TABLE XII. ESC16 total cross sections $\Xi^{-} p \rightarrow$ $\Lambda \Lambda, \Xi^{0} n, \Xi^{-} p, \Sigma^{0} \Lambda, \Sigma^{0} \Sigma^{0}, \Sigma^{-} \Sigma^{+}$in (mb) as a function of the beam particle laboratory beam particle momentum $p_{\text {lab }}$ in $(\mathrm{MeV} / c)$.

\begin{tabular}{lrrrrrc}
\hline \hline$p_{\Xi^{-}}$ & \multicolumn{1}{c}{$\Lambda \Lambda$} & \multicolumn{1}{c}{$\Xi^{0} n$} & \multicolumn{1}{c}{$\Xi^{-} p$} & $\Sigma^{0} \Lambda$ & $\Sigma^{0} \Sigma^{0}$ & $\Sigma^{-} \Sigma^{+}$ \\
\hline 10 & 928.53 & 1049.99 & 744.15 & - & - & - \\
50 & 52.54 & 45.94 & 12.29 & - & - & - \\
100 & 19.27 & 19.97 & 7.93 & - & - & - \\
200 & 7.25 & 12.12 & 7.39 & - & - & - \\
300 & 4.04 & 10.27 & 8.19 & - & - & - \\
400 & 2.71 & 9.29 & 9.23 & - & - & - \\
500 & 2.04 & 8.57 & 10.10 & - & - & - \\
550 & 1.83 & 8.28 & 10.10 & - & - & - \\
600 & 1.66 & 7.89 & 9.95 & 2.17 & - & - \\
700 & 1.45 & 7.39 & 12.17 & 2.91 & - & - \\
800 & 1.35 & 6.87 & 13.91 & 3.06 & - & - \\
900 & 1.35 & 6.33 & 15.48 & 3.07 & - & - \\
950 & 1.39 & 6.07 & 16.26 & 3.07 & - & - \\
1000 & 1.46 & 5.74 & 17.05 & 2.97 & 0.04 & 0.30 \\
\hline \hline
\end{tabular}
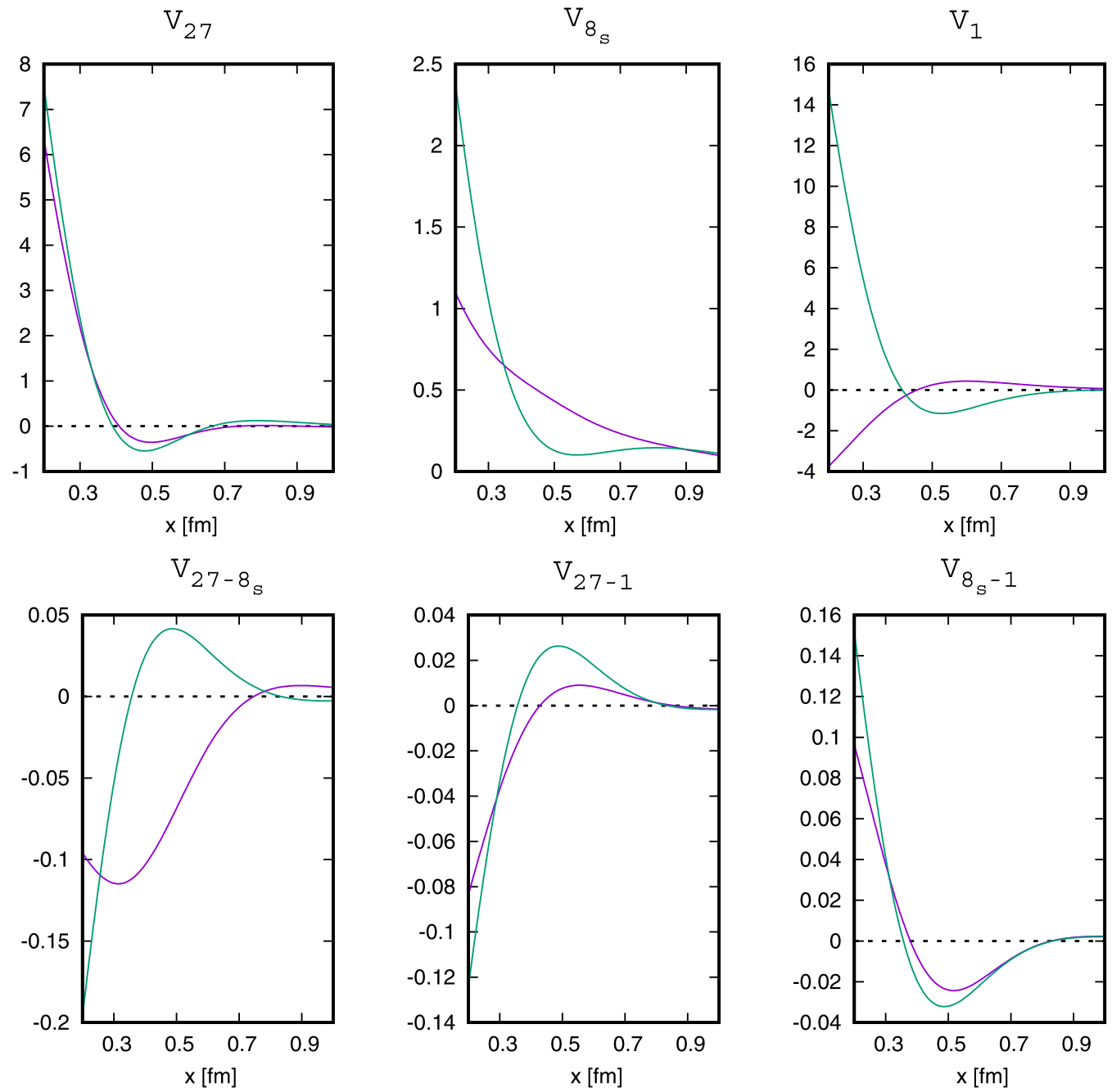

FIG. 14. Potentials in the symmetric SU(3)-irrep base. The red and green lines denote potentials with and without SU(3)-symmetry breaking respectively. The units of the vertical axes are $(\mathrm{GeV})$. 
TABLE XIII. ESC16 $L=0,1,2$ partial-wave total cross sections $\Xi^{-} p \rightarrow \Lambda \Lambda, \Xi^{0} n, \Xi^{-} p, \Sigma^{0} \Lambda$ in (mb) as a function of the beam particle laboratory beam particle momentum $p_{\text {lab }}=500 \mathrm{MeV} / c . \sigma_{s}$ and $\sigma_{t}$ are the spin-singlet and spin-triplet cross sections.

\begin{tabular}{|c|c|c|c|c|c|c|c|c|c|}
\hline \multirow[b]{2}{*}{$L$} & \multicolumn{3}{|c|}{$\Xi^{-} p \rightarrow \Lambda \Lambda$} & \multicolumn{3}{|c|}{$\Xi^{-} p \rightarrow \Xi^{0} n$} & \multicolumn{3}{|c|}{$\Xi^{-} p \rightarrow \Xi^{-} p$} \\
\hline & $\sigma_{s}$ & $\sigma_{t}$ & $\sigma_{T}$ & $\sigma_{s}$ & $\sigma_{t}$ & $\sigma_{T}$ & $\sigma_{s}$ & $\sigma_{t}$ & $\sigma_{T}$ \\
\hline 0 & 1.58 & - & 1.58 & 0.11 & 5.64 & 5.75 & 5.63 & 3.47 & 9.10 \\
\hline 1 & - & 0.41 & 0.41 & 0.04 & 2.31 & 2.35 & 0.20 & 0.72 & 0.92 \\
\hline 2 & 0.05 & - & 0.05 & 0.06 & 0.41 & 0.47 & 0.01 & 0.07 & 0.08 \\
\hline Tot & 1.63 & 0.41 & 2.04 & 0.21 & 8.36 & 8.57 & 5.84 & 4.26 & 10.10 \\
\hline Exp & & $4.3_{-2.7}^{+6.3}$ & & & $\leqslant 10$ & & & $\leqslant 24$ & \\
\hline
\end{tabular}

In Table $\mathrm{X}$ the $I=0$ total cross sections for $\Lambda \Lambda \rightarrow$ $\Lambda \Lambda, \Xi N$ are shown as a function of the beam laboratory momentum $p_{\text {lab }}=p_{\Lambda}$. Being dominantly $S$ wave, there is in principle a (sharp) cusp at the $\Xi N$ threshold, i.e., $p_{\Lambda}=344.4$ $\mathrm{MeV} / c$, which indeed is visible in the table. In Table $\mathrm{X}$ we also show the $\Xi N \rightarrow \Xi N, \Lambda \Lambda$ total cross sections as a function of the laboratory momentum $p_{\Xi}$. In Table XI the $I=1$ total cross sections for the $\Xi N \rightarrow \Xi N, \Sigma \Lambda$ and the $I=1, L=0 \Sigma \Lambda \rightarrow \Sigma \Lambda, \Xi N, \Sigma \Sigma$ reactions are given as a function of the laboratory momentum $p_{\mathrm{Lab}}=p_{\Xi}$. Similarly for $\Xi^{-} p$ in Table XII. In the KEK-224 experiment for $p_{\Xi}=$ $500 \mathrm{MeV} / c$ the upper limit for the total elastic cross section $\sigma_{e l}\left(\Xi^{-} p\right)=24 \mathrm{mb}$ at $90 \%$ confidence level [29]. Because the $\Xi^{-} p$ is a combination of the isospin states with factors $1 / \sqrt{2}$ the total cross sections in Tables X and XI are divided by 4 .

In Table XIII the $\Xi^{-} p$ cross sections are shown for the ESC16-model parameter set. In the measurements at $p_{\Xi}=$ $500 \mathrm{MeV} / c$ [30] for the $\Xi^{-} p \rightarrow \Lambda \Lambda$ it was found to be $4.3_{-2.7}^{+6.3} \mathrm{mb}$, which compares reasonably well with the value $2.01 \mathrm{mb}$ in the table. For the elastic $\Xi^{-} p$ cross section we have 9.34 which is clearly consistent with the upper limit of 24 $\mathrm{mb}$. Also, the cross section $8.37 \mathrm{mb}$ for the inelastic reaction $\Xi^{-} p \rightarrow \Xi^{0} n$ is compatible with the estimate of $10 \mathrm{mb}$ in [30]. We note that the total cross sections are rather constant in the range $400 \leqslant p_{\Xi^{-}} \leqslant 1000 \mathrm{MeV} / c$. Table XIII gives for model ESC16 the contributions from the partial waves for $L=0,1,2$ and the total cross sections.
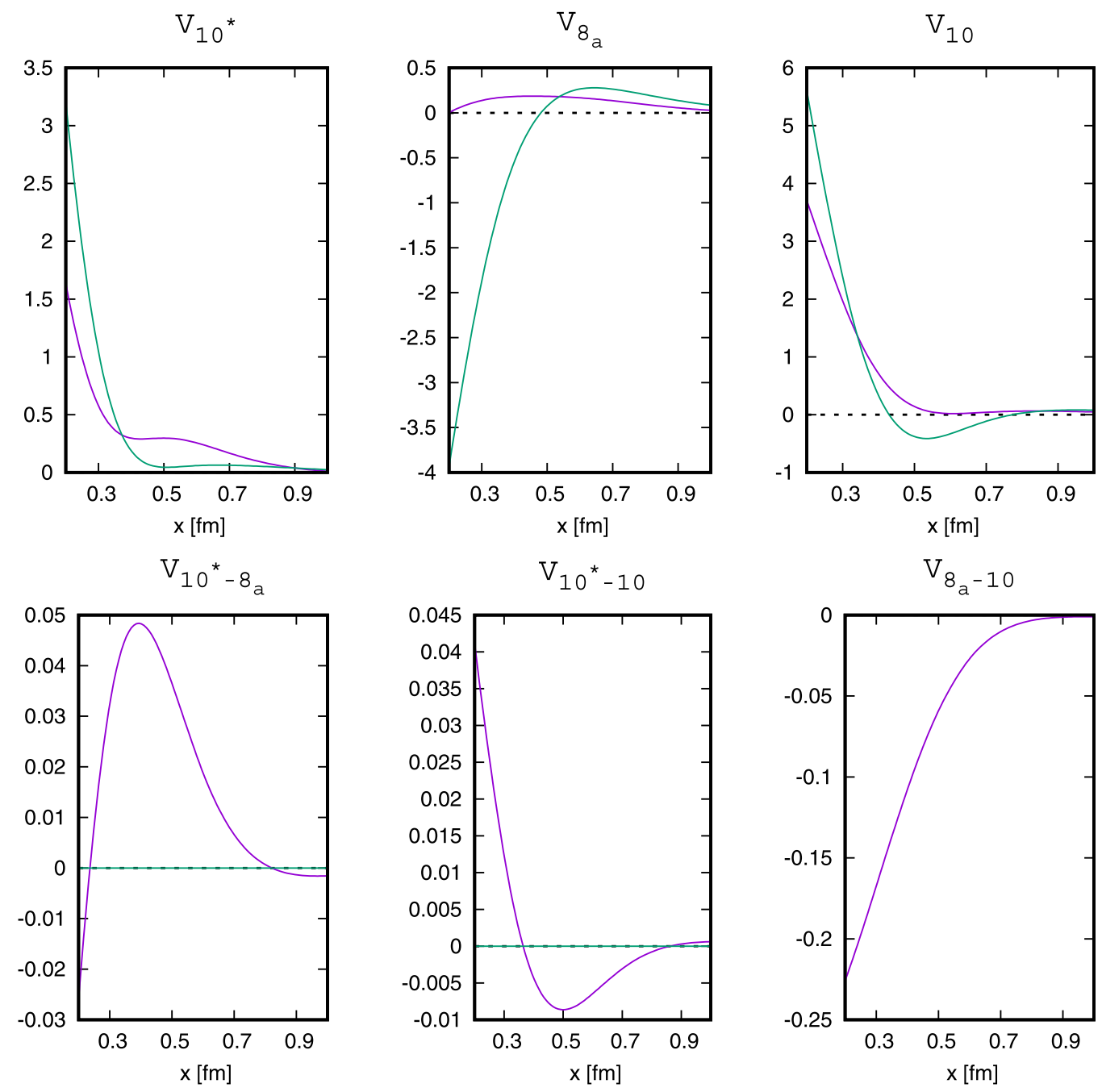

FIG. 15. Potentials in the antisymmetric SU(3)-irrep base. The red and green lines denote potentials with and without SU(3)-symmetry breaking, respectively. The units of the vertical axes are $(\mathrm{GeV})$. 


\section{E. Flavor SU(3)-irrep potentials}

In Figs. 14 and 15 the potentials in the SU(3)-irrep based states are displayed. In the upper and lower panels the diagonal and the nondiagonal potentials are shown, respectively. Notice that the nondiagonal potentials are considerably smaller than the diagonal ones, which shows the approximate SU(3) symmetry. The red/solid lines show averages of the SU(3)-irrep potentials using the potentials on the particle basis. The green/dashed lines are the irrep potentials in an SU(3) limit, where $M_{N}=M_{\Lambda}=M_{\Sigma}=M_{\Xi}=1115.6 \mathrm{MeV}, m_{\pi}=$ $m_{K}=m_{\eta}=m_{\eta^{\prime}}=400 \mathrm{MeV}, m_{\rho}=m_{K^{*}}=m_{\omega}=m_{\phi}=800$ $\mathrm{MeV}$, and $m_{a 0}=m_{\kappa}=m_{\sigma}=m_{f_{0}^{\prime}}=880 \mathrm{MeV}$. These values for the masses are close to the GMO [49] octet and singlet masses 410 and $885 \mathrm{MeV}$ for the pseudoscalar and vector mesons respectively. The cut-off masses for pseudoscalar, vector, and axial vector have been set equal to the octet ones, i.e., $\Lambda_{1}^{P}=\Lambda_{8}^{P}$ etc. But, for the scalar nonet $\Lambda_{8}^{S}=\Lambda_{1}^{S}$.

Comparison with the results from LQCD [50,51] shows qualitatively very similar results. The exception is the SU(3)singlet $\{1\}$ irrep. Here, LQCD potential is attractive for $0<$ $r<\infty$, whereas in ESC16 there is an attractive pocket for $r \leqslant 0.5 \mathrm{fm}$ and is repulsive for $r>0.5 \mathrm{fm}$. This shape is due to the behavior of the spin-spin potentials from pseudoscalar and vector exchange, which have zero volume integrals. In the $\{1\}$ irrep for the SU(3)-broken potential (solid line) there is no bound state, i.e., no $\mathrm{H}$ particle [52]. This is in agreement with the recent experimental result studying $\Upsilon(1 S, 2 S)$ decay [53].

\section{VII. $\Xi N$ G-MATRIX INTERACTION AND $\Xi$-NUCLEUS STATES}

\section{A. $G$-matrix interaction}

We calculate $\Xi$ potential energies $U_{\Xi}$ and derive $\Xi N$ $G$-matrix interactions $G_{\Xi N}(r)$ in nuclear matter with use of ESC16. ${ }^{1}$ For comparison, $G$-matrix calculations are performed for the HAL-QCD $\Xi N$ potential based on the lattice QCD [15]. Since the HAL-QCD potential was not determined uniquely and the three versions $(\mathrm{t} 11, \mathrm{t} 12, \mathrm{t} 13)$ were proposed, we use here the $t 13$ version giving the reasonable attraction in comparison with experimental data.

$G$-matrix calculations are performed with the continuous $(\mathrm{CON})$ choice, where off-shell potentials are taken into account continuously from on-shell ones in intermediate propagations of correlated pairs. A two-body ${ }^{(2 T+1)(2 S+1)} L_{J}$ state is specified by spin $S$, isospin $T$, orbital, and total angular momenta $L$ and $J$, respectively. Imaginary parts of $G$ matrices appear due to energy-conserving transitions from $\Xi N$ to $\Lambda \Lambda$ channels in ${ }^{11} S_{0}$ and ${ }^{13} P_{J}$ states. A conversion width $\Gamma_{\Xi}^{c}$ is obtained from an imaginary part of $U_{\Xi}$ by multiplying -2 .

The BNL-E885 experiment [19] suggests that a $\Xi^{-}$single particle potential in ${ }_{\Xi^{-}}^{12} \mathrm{Be}$ is given by the attractive WoodsSaxon (WS) potential with the depth $\approx-14 \mathrm{MeV}$. Recently, in the $\mathrm{E} 05{ }^{12} \mathrm{C}\left(K^{-}, K^{+}\right)$experiment at JPARC [54], some enhancement of cross sections has been observed in the $\Xi^{-}$

\footnotetext{
${ }^{1}$ In this section we denote isospin by $T$, the nuclear physics notation.
}

TABLE XIV. $U_{\Xi}\left(\rho_{0}\right)$ and partial wave contributions in ${ }^{(2 T+1)(2 S+1)} L_{J}$ states for ESC16 and ESC16+V $V_{\text {eff }}(\mathrm{A} 1, \mathrm{~A} 2, \mathrm{~B} 1, \mathrm{~B} 2)$ calculated with the CON choice. $\Gamma_{\Xi}^{c}$ denotes $\Xi N-\Lambda \Lambda$ conversion width. Also the calculated values are given for the HAL-QCD potential [15]. All entries are in $\mathrm{MeV}$.

\begin{tabular}{lrrrrrr}
\hline \hline & ESC16 & A1 & A2 & B1 & B2 & HAL-QCD \\
\hline$X_{2}$ & 0.0 & 2.85 & 2.55 & 1.65 & 1.07 & \\
$X_{3}$ & 0.0 & 0.0 & 1.6 & 0.0 & 3.0 & \\
$X_{s}$ & 0.0 & 0.0 & 0.0 & 10. & 10. & \\
${ }^{11} S_{0}$ & 2.1 & 1.4 & 1.4 & -4.0 & -4.0 & -4.9 \\
${ }^{13} S_{1}$ & -0.4 & -2.2 & -2.2 & -2.8 & -2.8 & -2.2 \\
${ }^{11} P_{1}$ & -0.2 & -0.3 & -0.3 & -0.3 & -0.3 & \\
${ }^{13} P_{0}$ & -5.3 & -3.5 & -3.5 & -2.0 & -2.0 & \\
${ }^{13} P_{1}$ & 1.5 & 1.3 & 1.3 & 1.7 & 1.7 & \\
${ }^{13} P_{2}$ & -1.2 & -1.2 & -1.2 & -2.3 & -2.3 & \\
${ }^{31} S_{0}$ & 9.2 & 9.9 & 9.9 & 6.8 & 6.8 & 1.8 \\
${ }^{33} S_{1}$ & 7.6 & -13.5 & -13.9 & -4.7 & -4.9 & -5.4 \\
${ }^{31} P_{1}$ & 1.0 & 1.3 & 1.3 & 1.0 & 1.0 & \\
${ }^{33} P_{0}$ & 0.8 & 1.0 & 1.0 & 0.8 & 0.7 & \\
${ }^{33} P_{1}$ & -2.0 & -2.8 & -2.8 & -3.0 & -3.0 & \\
${ }^{33} P_{2}$ & 0.5 & 0.1 & 0.1 & -1.0 & -1.0 & \\
$U_{\Xi}$ & +13.7 & -8.5 & -9.0 & -10.1 & -10.4 & -10.6 \\
$\Gamma_{\Xi}^{c}$ & 5.1 & 5.7 & 5.7 & 0.5 & 0.5 & 0.2 \\
\hline \hline
\end{tabular}

bound-state region, suggesting possible existing of a $1 S$ bound state. Furthermore, as discussed later, the emulsion events of $\Xi$ hypernuclei give clear evidences for attractive $\Xi$-nucleus potentials. Here, it should be noted that the depth of the above WS potential is related to the potential energy $U_{\Xi}\left(\rho_{0}\right)$ in normal-density matter, when a $\Xi$-nucleus potential is modeled simply as a local-density potential $U_{\Xi}(\rho(r))$. Though such a potential is not adopted here and their relation is only indirect, it is clear that the repulsive value of $U_{\Xi}\left(\rho_{0}\right)$ for ESC16 in Table XIV is in contradiction with the above-mentioned experimental indications of attractive $\Xi$-nucleus interactions.

In order to realize an attractive $\Xi$-nucleus interaction, an effective $\Xi N$ interaction $V_{\text {eff }}$ composed of two terms, a $(\pi \omega)$ pair exchange interaction $V_{\pi \omega}$ and a $\mathrm{SU}(3)$-singlet interaction $V_{\text {singlet }}$, is added phenomenologically to the ESC16 interaction. The $V_{\pi \omega}$ spin-spin and tensor potential functions are given in Ref. [40], Eq. (C17). The effective interaction is given as

$$
\begin{aligned}
V_{\text {eff }}(r ; \rho) & =\left(X_{2}+X_{3} f(\tilde{\rho})\right) V_{\pi \omega}(r)+X_{s} V_{\text {singlet }}(r), \\
f(\tilde{\rho}) & =\tilde{\rho} \exp (-\eta \tilde{\rho})
\end{aligned}
$$

with $\tilde{\rho}=\rho / \rho_{0}$. Here, the first term is assumed to have density-dependent strength, where $f(\tilde{\rho})$ is modeled so that it dominates at low density and is negligible at higher densities. $X_{2}$ and $X_{3}$ are strengths of density-independent two-body part and density-dependent one. This term brings about a large attraction especially in $(T S P)=(11+)$ state, $P$ denoting parity. $X_{S}$ is the strength of the SU(3)-singlet interaction, which works in $(T S P)=(00+)$ and $(01-)$ states. The form of the singlet potential is chosen as $V_{\text {singlet }}=$ $-m_{s} \exp \left(-m_{s}^{2} r^{2} / 4\right), m_{s}=760 \mathrm{MeV}$ and for the different BB channels the weights are the coefficients of $V_{1}$ in Table XXVI. 
Parameters $X_{2}, X_{3}, \eta$, and $X_{s}$ are determined so that the derived $G$-matrix interaction derived from $\mathrm{ESC} 16+V_{\text {eff }}$ reproduces the experimental value of $\Xi^{-}$binding energies $B_{\Xi^{-}}$ in the $\Xi^{-}+{ }^{14} \mathrm{~N}$ system $[10,55]$. We give here the four parameter sets $\mathrm{A} 1, \mathrm{~A} 2, \mathrm{~B} 1$, and $\mathrm{B} 2$ specified by $\left(X_{2}, X_{3}, X_{s}\right)=$ $(2.85,0.0,0.0),(2.55,1.60,0.0),(1.65,0.0,10.0)$, and $(1.07$, $3.00,10.0)$, respectively, $\eta$ being taken as 1.6 . In the case of $\mathrm{B} 1$ and B2, the Pauli-blocking effect for [51] configuration is not taken into account in the ESC16 construction. As shown later, these four sets for $V_{\text {eff }}$ give reasonable values of $B_{\Xi^{-}}(1 S)$ and $B_{\Xi^{-}}(2 P)$ in the $\Xi^{-}+{ }^{14} \mathrm{~N}$ system.

Table XIV shows the potential energies $U_{\Xi}$ and their partial-wave contributions in ${ }^{(2 T+1)(2 S+1)} L_{J}$ states at normal density $\rho_{0}$ for ESC16, A1, A2, B1, and B2. The $U_{\Xi}$ value for ESC16 turns out to be repulsive. Comparing the partial-wave contributions for $\mathrm{ESC} 16$ with those for $\mathrm{A} 1$ or $\mathrm{A} 2$, one find that the main difference is in ${ }^{33} S_{1}$-state contributions, being repulsive in the former and strongly attractive in the latter. The negative value of $U_{\Xi}$ in the case of $\mathrm{A} 1$ or $\mathrm{A} 2$ is owing to the large ${ }^{33} S_{1}$ attractive contribution of the $\Xi N-\Lambda \Sigma-\Sigma \Sigma$ tensor-coupling term in the $(\pi \omega)$-pair exchange interaction in Eq. (7.1). In the case of $\mathrm{B} 1$ or B2, the ${ }^{33} S_{1}$ contributions are not so attractive, but instead the SU(3)-singlet interaction in the ${ }^{11} S_{0}$ state contributes attractively to give the negative value of $U_{\Xi}$. The calculated values of $\Gamma_{\Xi}^{c}\left(\rho_{0}\right)$ are given in the Table XIV, the dominant contributions of which come from the $\Lambda \Lambda-\Xi N-\Sigma \Sigma$ coupling interaction in the ${ }^{11} S_{0}$ state. Here, it should be noted that the $\Gamma_{\Xi}^{c}$ values for B1 and B2 are far smaller than those for $\mathrm{A} 1$ and $\mathrm{A} 2$.

The calculated values for the HAL-QCD potential (t13) are also given in Table XIV. Here, $P$-state values are lacking because only the $S$-state potentials are given in Ref. [15]. There appear the two distinct features in the HAL-QCD result: One is the strongly attractive value of partial-wave contribution in ${ }^{11} S_{0}$ state. It should be noted that the value in this state is given genuinely by the interaction because of the statistical weight factor $(2 T+1)(2 J+1)=1$. The other is the small value of $\Gamma_{\Xi}^{c}$. Concerning these features, B1/B2 are more similar to HAL-QCD than A1/A2 owing to the SU(3)-singlet interactions. For reference, let us show the calculated values of $U_{\Xi}\left(\rho_{0}\right)$ for the t12 and t11 versions of the HAL-QCD potential, being $-14.1 \mathrm{MeV}$ and $-16.2 \mathrm{MeV}$, respectively. Thus, these versions lead to overbinding values of $B_{\Xi}$ in comparison with the experimental data.

It is interesting to compare the present results with those by the chiral NLO interactions. Their $G$-matrix results are given in Ref. [56], where their calculated values of $U_{\Xi}\left(\rho_{0}\right)$ and their partial-wave contributions can be found in Figs. 3 and 2 (the values at $k=0 \mathrm{fm}^{-1}$ in the figures), respectively. Then, their $U_{\Xi}\left(\rho_{0}\right)$ value is rather close to those for $\mathrm{A} 1 / \mathrm{A} 2$ and $\mathrm{B} 1 / \mathrm{B} 2$. The ${ }^{11} S_{0}$ and ${ }^{13} S_{1}\left({ }^{31} S_{0}\right.$ and $\left.{ }^{33} S_{1}\right)$ contributions are similar to those for B1/B2 (A1/A2).

Let us compare the features of $\mathrm{A} 1, \mathrm{~A} 2, \mathrm{~B} 1$, and $\mathrm{B} 2$ by showing the partial-wave contributions to $U_{\Xi}$ as a function of $k_{F}$. In Fig. 16, $S$ - and $P$-wave contributions, $U_{\Xi}(S)$ and $U_{\Xi}(P)$, are drawn by thick (thin) solid and short-dashed curves are for $\mathrm{A} 1$ and $\mathrm{A} 2$ (B1 and B2), respectively. For $U_{\Xi}(S)$, the curves for A1 and B1 (A2 and B2) are found to be similar with each

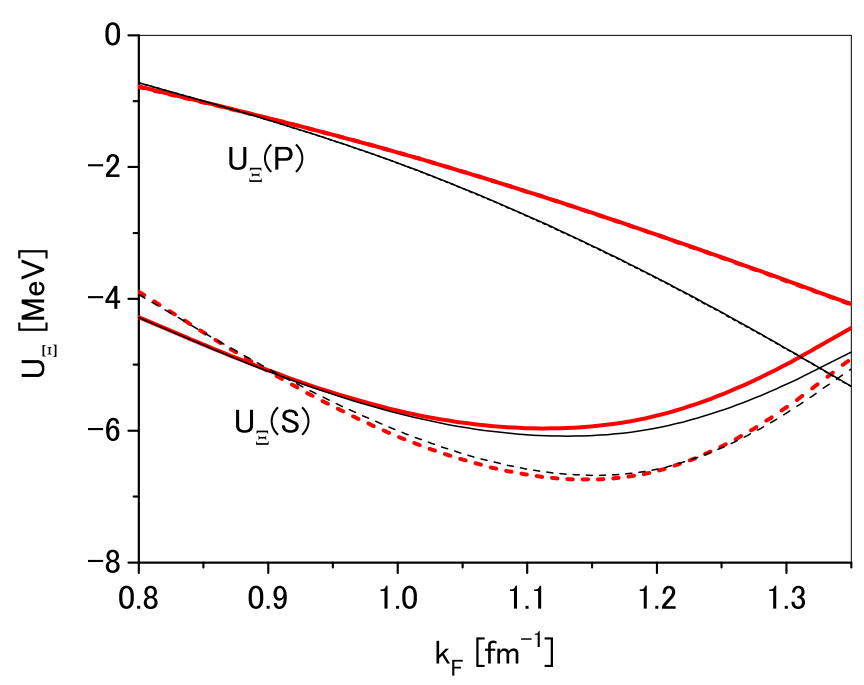

FIG. 16. $U_{\Xi}(S)$ and $U_{\Xi}(P)$, being $S$ - and $P$-wave contributions to $U_{\Xi}$, respectively, are drawn as a function of $k_{F}$. Thick (thin) solid and short-dashed curves are for A1 and A2 (B1 and B2), respectively.

other. For $U_{\Xi}(P)$, the curves for $\mathrm{A} 1$ and $\mathrm{A} 2$ (B1 and $\mathrm{B} 2$ ) are almost superposed.

In Fig. 17, partial-wave contributions of ${ }^{(2 T+1)(2 S+1)} L_{J}$ states are drawn as a function of $k_{F}$. Here, ${ }^{11} S_{0},{ }^{13} S_{1},{ }^{31} S_{0}$, and ${ }^{33} S_{1}$ contributions for A1 (B1) are given by thick (thin) solid, dashed, short-dashed, and dot-dashed curves, respectively. The similar figure can be obtained for A2 and B2. In the figure the ${ }^{33} S_{1}$ curve for $\mathrm{A} 1$ is found to be far below that for $\mathrm{B} 1$, the reason why is because the value of $X_{2}$ in $\mathrm{A} 1$ is larger than that in B1. On the other hand, the ${ }^{11} S_{0}$ and ${ }^{31} S_{0}$ curves for B1 are below those for A1, the reason of which is as follows: In the ${ }^{11} S_{0}$ curve, the SU(3)-singlet interaction is included in B1. In the ${ }^{31} S_{0}$ curve, the Pauli-blocking effect for the [51] configuration is not taken into account in B1.

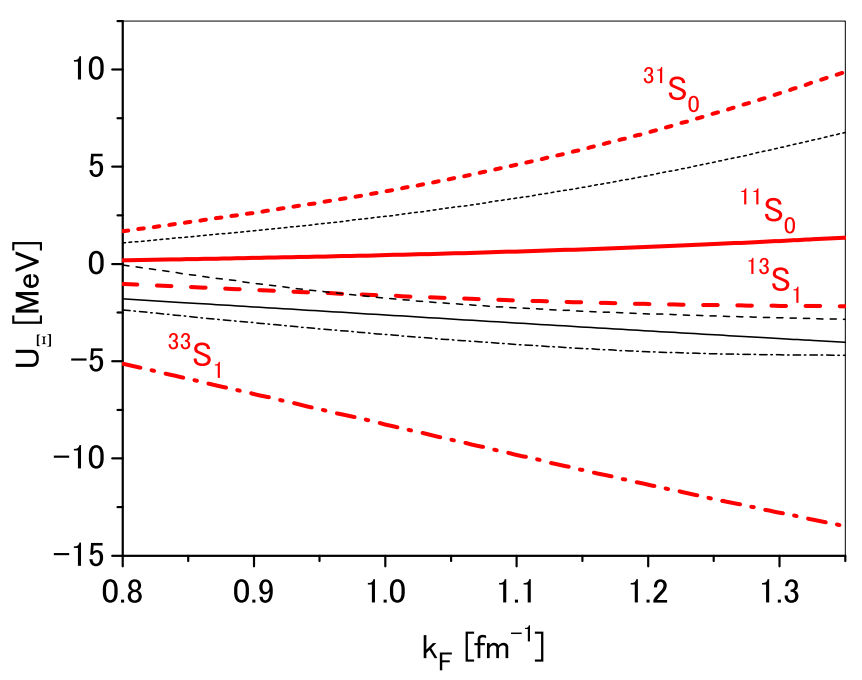

FIG. 17. Contributions of ${ }^{(2 T+1)(2 S+1)} L_{J}$ components to $U_{\Xi}$ are drawn as a function of $k_{F} \cdot{ }^{11} S_{0},{ }^{13} S_{1},{ }^{31} S_{0}$, and ${ }^{33} S_{1}$ contributions are given by thick (thin) solid, dashed, short-dashed, and dot-dashed curves for A1 (B1). 
TABLE XV. $\mathcal{G}\left(r ; k_{F}\right)=\left(a_{1}+b_{1} k_{F}+c_{1} k_{F}^{2}\right) \exp \left[-\left(r / \beta_{1}\right)^{2}\right]+$ $a_{2} \exp \left[-\left(r / \beta_{2}\right)^{2}\right]$ in ${ }^{(2 T+1)(2 S+1)} E$ and ${ }^{(2 T+1)(2 S+1)} O$ states with $\beta_{1}=0.9 \mathrm{fm}$ and $\beta_{2}=2.0 \mathrm{fm}$.

\begin{tabular}{|c|c|c|c|c|c|}
\hline & & $a_{1}$ & $b_{1}$ & $c_{1}$ & $a_{2}$ \\
\hline \multirow[t]{8}{*}{ A1 } & ${ }^{11} E$ & 82.68 & -33.36 & 19.73 & -5.448 \\
\hline & ${ }^{13} E$ & -55.49 & -1.213 & 19.13 & 0.364 \\
\hline & ${ }^{11} \mathrm{O}$ & 79.81 & 2.711 & 5.132 & -5.442 \\
\hline & ${ }^{13} \mathrm{O}$ & -1272 & 1289. & -379.7 & 0.356 \\
\hline & ${ }^{31} E$ & 49.99 & 1.493 & 19.08 & 0.514 \\
\hline & ${ }^{33} E$ & -99.34 & 62.12 & -8.145 & -1.603 \\
\hline & ${ }^{31} \mathrm{O}$ & 57.51 & -2.260 & 8.346 & 0.509 \\
\hline & ${ }^{33} \mathrm{O}$ & -10.92 & 6.496 & 4.247 & -1.598 \\
\hline \multirow[t]{8}{*}{$\mathrm{A} 2$} & ${ }^{11} E$ & 80.46 & -29.38 & 17.95 & -5.448 \\
\hline & ${ }^{13} E$ & -55.49 & -1.213 & 19.13 & 0.364 \\
\hline & ${ }^{11} \mathrm{O}$ & 79.81 & 2.711 & 5.132 & -5.442 \\
\hline & ${ }^{13} \mathrm{O}$ & -1411 & 1538. & -488.2 & 0.356 \\
\hline & ${ }^{31} E$ & 53.83 & -5.501 & 22.11 & 0.514 \\
\hline & ${ }^{33} E$ & 1.000 & -120.7 & 71.50 & -1.603 \\
\hline & ${ }^{31} \mathrm{O}$ & 56.82 & -.9798 & 7.783 & 0.509 \\
\hline & ${ }^{33} \mathrm{O}$ & 9.756 & -31.25 & 20.66 & -1.598 \\
\hline \multirow[t]{8}{*}{ B1 } & ${ }^{11} E$ & -466.5 & 490.8 & -148.5 & -5.394 \\
\hline & ${ }^{13} E$ & -65.87 & 4.220 & 17.41 & 0.373 \\
\hline & ${ }^{11} \mathrm{O}$ & 65.56 & 4.362 & 4.760 & -5.395 \\
\hline & ${ }^{13} \mathrm{O}$ & -529.4 & 298.5 & -23.77 & 0.373 \\
\hline & ${ }^{31} E$ & 30.54 & -4.355 & 18.45 & 0.517 \\
\hline & ${ }^{33} E$ & -30.45 & 8.704 & 8.253 & -1.583 \\
\hline & ${ }^{31} \mathrm{O}$ & 34.15 & -1.376 & 7.630 & 0.517 \\
\hline & ${ }^{33} \mathrm{O}$ & -48.81 & 7.115 & 5.125 & -1.583 \\
\hline \multirow[t]{8}{*}{ B2 } & ${ }^{11} E$ & -409.8 & 389.1 & -104.4 & -5.394 \\
\hline & ${ }^{13} E$ & -65.87 & 4.220 & 17.41 & 0.373 \\
\hline & ${ }^{11} \mathrm{O}$ & 65.56 & 4.362 & 4.760 & -5.395 \\
\hline & ${ }^{13} \mathrm{O}$ & -604.4 & 432.9 & -82.03 & 0.373 \\
\hline & ${ }^{31} E$ & 36.17 & -14.55 & 22.89 & 0.517 \\
\hline & ${ }^{33} E$ & 46.33 & -130.5 & 68.80 & -1.583 \\
\hline & ${ }^{31} \mathrm{O}$ & 31.33 & 3.750 & 5.405 & 0.517 \\
\hline & ${ }^{33} \mathrm{O}$ & -21.27 & -42.81 & 26.84 & -1.583 \\
\hline
\end{tabular}

For applications to finite $\Xi$ systems, $\Xi N-\Xi N$ central parts of complex $G$-matrix interactions are represented in two-range Gaussian forms, whose coefficients are given as a function of $k_{F}$ :

$$
\begin{aligned}
\mathcal{G}\left(r ; k_{F}\right)= & \left(a_{1}+b_{1} k_{F}+c_{1} k_{F}^{2}\right) \exp \left[-(r / 0.9)^{2}\right] \\
& +a_{2} \exp \left[-(r / 2.0)^{2}\right]
\end{aligned}
$$

in ${ }^{(2 T+1)(2 S+1)} E$ and ${ }^{(2 T+1)(2 S+1)} O$ states. The determined parameters for $\mathrm{A} 1, \mathrm{~A} 2, \mathrm{~B} 1$, and $\mathrm{B} 2$ are given in Table XV. Imaginary parts are represented as $\mathcal{G}_{i m}\left(r ; k_{F}\right)=\left(a_{1}+b_{1} k_{F}+\right.$ $\left.c_{1} k_{F}^{2}\right) \exp \left[-(r / 0.9)^{2}\right]$ in ${ }^{11} E$ and ${ }^{13} O$ states, whose parameters are in Table XVI.

Also the $G$-matrix interactions derived from the HAL-QCD potential are represented similarly in two-range Gaussian forms for applications to finite $\Xi$ systems.

\section{B. Experimental information}

Experimentally, the most important information for $\Xi N$ interactions has been obtained from emulsion events of simul-
TABLE XVI. Imaginary parts: $\mathcal{G}_{i m}\left(r ; k_{F}\right)=\left(a_{1}+b_{1} k_{F}+\right.$ $\left.c_{1} k_{F}^{2}\right) \exp \left[-(r / 0.9)^{2}\right]$ in ${ }^{11} E$ and ${ }^{13} O$ states.

\begin{tabular}{lcccc}
\hline \hline & & $a_{1}$ & $b_{1}$ & $c_{1}$ \\
\hline A1 & ${ }^{11} E$ & -87.66 & 17.11 & -.3357 \\
& ${ }^{13} O$ & -579.4 & 746.0 & -251.7 \\
A2 & ${ }^{11} E$ & -69.75 & -15.57 & 13.88 \\
& ${ }^{13} O$ & -696.1 & 953.9 & -341.9 \\
B1 & ${ }^{11} E$ & 1.284 & -3.106 & -.3416 \\
& ${ }^{13} O$ & -48.48 & 26.17 & .6170 \\
B2 & ${ }^{11} E$ & 5.001 & -9.872 & 2.601 \\
& ${ }^{13} O$ & -66.19 & 57.90 & -13.16 \\
\hline \hline
\end{tabular}

taneous emission of two $\Lambda$ hypernuclei (twin $\Lambda$ hypernuclei) from a $\Xi^{-}$absorption point: The $\Xi^{-}$hyperon produced by the $\left(K^{-}, K^{+}\right)$reaction is absorbed into a nucleus $\left({ }^{12} \mathrm{C},{ }^{14} \mathrm{~N}\right.$, or ${ }^{16} \mathrm{O}$ in emulsion) from some atomic orbit, and two $\Lambda$ hypernuclei are produced by the secondary $\Xi^{-} p \rightarrow \Lambda \Lambda$ process. Then, the energy difference between the initial $\Xi^{-}$state and the final twin $\Lambda$ state gives rise to the binding energy $B_{\Xi^{-}}$between $\Xi^{-}$ and the nucleus. It is well known that capture probabilities of $\Xi^{-}$from $2 P$ states are far smaller than those from $3 D$ states. In spite of this fact, twin $\Lambda$ hypernuclei are produced dominantly after $2 P-\Xi^{-}$captures. As discussed in Ref. [57], the reason is because sticking probabilities of two $\Lambda$ 's produced after $2 P$ $\Xi^{-}$captures are substantially larger than those after $3 D-\Xi^{-}$ captures.

Two events of twin $\Lambda$ hypernuclei (I) [58] and (II) [59] were observed in the KEK E-176 experiment, and the event (III) [10] was in the KEK E373 experiment. In the cases of (I) and (II), each event has no unique interpretation for its reaction process. However, it is possible to find a consistent understanding for these two events as follows: The events (I) and (II) were interpreted to be reactions of $\Xi^{-}$captured by ${ }^{12} \mathrm{C}$. Assuming that the $\Xi^{-}$is absorbed from the $2 P$ orbit in each case, we have consistently the following reactions:

$$
\begin{aligned}
& \text { (I) } \Xi^{-}+{ }^{12} \mathrm{C} \rightarrow{ }_{\Lambda}^{9} \mathrm{Be}+{ }_{\Lambda}^{4} \mathrm{H}\left(B_{\Xi^{-}}=0.82 \pm 0.17 \mathrm{MeV}\right), \\
& \text { (II) } \Xi^{-}+{ }^{12} \mathrm{C} \rightarrow{ }_{\Lambda}^{9} \mathrm{Be}^{*}+{ }_{\Lambda}^{4} \mathrm{H}\left(B_{\Xi^{-}}=0.82 \pm 0.14 \mathrm{MeV}\right) .
\end{aligned}
$$

The event (III) is uniquely identified as

$$
\Xi^{-}+{ }^{14} \mathrm{~N} \rightarrow{ }_{\Lambda}^{10} \mathrm{Be}+{ }_{\Lambda}^{5} \mathrm{He}
$$

being the clear evidence of a $\Xi^{-}+{ }^{14} \mathrm{~N}$ bound state. The $B_{\Lambda}$ value of ${ }_{\Lambda}^{10} \mathrm{Be}$ in the ground state was determined as $8.60 \pm 0.07 \mathrm{MeV}$ by the $\left(e, e^{\prime} K^{+}\right)$experiment JLab E05-115 [60]. Then, we have $B_{\Xi^{-}}=3.87 \pm 0.13 \mathrm{MeV}$ using this value of $B_{\Lambda}\left({ }_{\Lambda}^{10} \mathrm{Be}\right)$. It is possible very much, however, that the ${ }_{\Lambda}^{10} \mathrm{Be}$ produced in the event (III) is in some excited state. The experimental spectra of ${ }_{\Lambda}^{10} \mathrm{Be}$ [60] shows that only the first excited state leads to a positive value. In this case, we have $B_{\Xi^{-}}=1.11 \pm 0.25 \mathrm{MeV}$.

Recently, the J-PARC E07 experiment has been performed and the analysis is now in progress, where many events of twin- $\Lambda$ hypernuclei in emulsion have been observed. In the cases of $\Xi^{-}+{ }^{14} \mathrm{~N}$ events, they have found not only the $2 P$ - 
$\Xi^{-}$states $\left(B_{\Xi}=1.0-1.5 \mathrm{MeV}\right)$ but also the $1 S-\Xi^{-}$states $\left(B_{\Xi}=6.27 \pm 0.32 \mathrm{MeV}\right)[55]$.

\section{Results for finite $\Xi$ systems}

As demonstrated in Ref. [21,61], the observed spectra of $\Lambda, \Xi$ hypernuclei are described successfully with the $\Lambda N$ - and $\Xi N$-nucleus folding potentials derived from the $\Lambda N$ and $\Xi N$ $G$-matrix interactions. Here, the same method is applied to $\Xi^{-}$-nucleus systems. A $\Xi$-nucleus folding potential in a finite system is obtained from $\mathcal{G}_{( \pm)}^{T S}\left(r ; k_{F}\right)$ as follows:

$$
\begin{aligned}
U_{\Xi}\left(\mathbf{r}, \mathbf{r}^{\prime}\right)= & U_{d r}+U_{e x} \\
U_{d r}= & \delta\left(\mathbf{r}-\mathbf{r}^{\prime}\right) \int d \mathbf{r}^{\prime \prime} \rho\left(\mathbf{r}^{\prime \prime}\right) V_{d r}\left(\left|\mathbf{r}-\mathbf{r}^{\prime \prime}\right| ; k_{F}\right) \\
U_{e x}= & \rho\left(\mathbf{r}, \mathbf{r}^{\prime}\right) V_{e x}\left(\left|\mathbf{r}-\mathbf{r}^{\prime}\right| ; k_{F}\right) \\
\left(\begin{array}{c}
V_{d r} \\
V_{e x}
\end{array}\right)= & \frac{1}{2\left(2 t_{Y}+1\right)\left(2 s_{Y}+1\right)} \sum_{T S}(2 T+1)(2 S+1) . \\
& \times\left[\mathcal{G}_{( \pm)}^{T S} \pm \mathcal{G}_{(\mp)}^{T S}\right]
\end{aligned}
$$

where $( \pm)$ denote parity quantum numbers. Here, it is assumed that a core nucleus is spherical and its spin and isospin are zero. Densities $\rho(r)$ and mixed densities $\rho\left(r, r^{\prime}\right)$ are obtained from Skyrme-HF wave functions. Using a local approximation for $U_{e x}$, we have the local $\Xi$-nucleus potential $U_{\Xi}\left(r ; k_{F}\right)$ for a spherical core. The isospin-dependence of $\mathcal{G}_{( \pm)}^{T S}\left(r ; k_{F}\right)$ leads to the Lane term. In this work, only the diagonal parts of the $\mathbf{t}_{\Xi} \mathbf{T}_{\mathbf{c}}$ term are taken into account. The $T S$ dependences of $\mathcal{G}_{( \pm)}^{T S}\left(r ; k_{F}\right)$ are considerably different between $\mathrm{A} 1$ and B1 (A2 and B2). These differences do not appear in the following cases of core nuclei.

For $k_{F}$ included in $\mathcal{G}\left(r ; k_{F}\right)$, we use the averaged-density approximation (ADA), where $k_{F}$ values are obtained from averaged values of $\rho(r): \bar{\rho}=\left\langle\phi_{Y}(r)|\rho(r)| \phi_{Y}(r)\right\rangle$ with a hyperon wave function $\phi_{Y}(r)$. In the case of $\Lambda$ hypernuclei, the ADA gives rise to good fitting to the experimental spectra.

Table XVII shows the results for $1 S$ and $2 P(1 S, 2 P$, and $3 D$ ) bound states in $\Xi^{-}+{ }^{12} \mathrm{C}, \Xi^{-}+{ }^{14} \mathrm{~N}$, and $\Xi^{-}+{ }^{16} \mathrm{O}$ $\left(\Xi^{-}+{ }^{27} \mathrm{Al}\right)$ systems in the cases of using $\mathrm{A} 1, \mathrm{~A} 2, \mathrm{~B} 1$, and $\mathrm{B} 2$, where Coulomb interactions between $\Xi^{-}$and nuclear cores are taken into account. $B_{\Xi^{-}}$and $\sqrt{\left\langle r^{2}\right\rangle}$ are binding energy and rms radius of $\Xi^{-}$, respectively. Conversion widths $\Gamma_{\Xi^{-}}^{c}$ come from the imaginary potentials in ${ }^{11} S_{1}$ and ${ }^{13} P$ states. $2 P-\Xi^{-}$states in ${ }^{12} \mathrm{C},{ }^{14} \mathrm{~N}$, and ${ }^{16} \mathrm{O}$, and $3 D-\Xi^{-}$states in ${ }^{27} \mathrm{Al}$, marked by $(*)$ in the table, are so-called Coulomb-assisted bound states (CABS), which means that they cannot be bound without attractive Coulomb interactions. The values of $\sqrt{\left\langle r^{2}\right\rangle}$ for CABS are far smaller than those for pure Coulomb bound states.

The calculated values of $B_{\Xi^{-}}(2 P)$ in $\Xi^{-}+{ }^{12} \mathrm{C}$ system are $0.7-1.1 \mathrm{MeV}$ for $\mathrm{A} 1, \mathrm{~A} 2, \mathrm{~B} 1$, and $\mathrm{B} 2$, being consistent with the experimental value $0.82 \pm 0.14 \mathrm{MeV}$. In $\Xi^{-}+{ }^{14} \mathrm{~N}$ system, the calculated values of $B_{\Xi^{-}}(1 S)$ and $B_{\Xi^{-}}(2 P)$ are 5.1-5.7 MeV and 1.2-1.4 MeV, respectively, for A1, A2, B1, and $\mathrm{B} 2$. These values are consistent with the recent observations that $B_{\Xi^{-}}(2 P)=1-1.5 \mathrm{MeV}$ and $B_{\Xi^{-}}(1 S)=5-7 \mathrm{MeV}$ [55]. Let us remark here the difference $B_{\Xi^{-}}(1 S)-B_{\Xi^{-}}(2 P)$ in the $\Xi^{-}+{ }^{14} \mathrm{~N}$ case: The calculated values are $3.7(3.8) \mathrm{MeV}$ for A1 (B1) and 4.5 (4.4) $\mathrm{MeV}$ for A2 (B2), respectively. The reason why the latter values are larger than the former values is because the density-dependent term $X_{3} f(\tilde{\rho}) V_{\pi \omega}(r)$ in Eq. (7.1) strengthen the $k_{F}$ dependence of the $G$-matrix interaction Eq. (7.2).

In Table XVII, it is noted that the results for A1 and B1 (A2 and B2) are similar with each other. This is because the different (TS) dependence of A1 and B1 (A2 and B2) are not revealed in the present systems that spin and isospin of nuclear cores are zero. The differences between A1 (B1) and A2 (B2) come from the density-dependent term $X_{3} f(\tilde{\rho}) V_{\pi \omega}(r)$ in Eq. (7.1). The most striking difference between A1 and B1 (A2 and B2) can be seen in the conversion widths: The calculated values of $\Gamma_{\Xi^{-}}^{c}$ for B1 (B2) are far smaller than those for A1 (A2), because the ${ }^{11} S_{0} \Xi N-\Lambda \Lambda$ coupling interaction in the former is far weaker than that in the latter.

The results for the HAL-QCD potential also are included in Table XVII. The calculated values of $B_{\Xi}(1 S)$ are more or less similar to those for the ESC models A1/A2 and B1/B2. On the other hand, the values of $B_{\Xi}(2 P, 3 D)$ are systematically smaller than those for the ESC models, for which there are two possible reasons: The one is that the density $\left(k_{F}\right)$ dependence of the $G$-matrix interaction is weak, because in the HAL-QCD potential $\Xi N-\Lambda \Sigma-\Sigma \Sigma$ coupling interactions are renormalized into the $\Xi N-\Xi N$ single-channel potentials. The other is that $p$-state interaction (possibly attractive) is missing in the HALQCD potential for the present.

$\Xi^{-}$bound states in ${ }^{12} \mathrm{C},{ }^{14} \mathrm{~N}$, and ${ }^{16} \mathrm{O}$ are expected to be observed soon as emulsion events in J-PARC E07 experiment. $\Xi^{-}+{ }^{27} \mathrm{Al}$ bound states are expected to be observed in ${ }^{28} \mathrm{Si}\left(K^{-}, K^{+}\right)$reactions.

Recapitulating the results for the various $V_{\text {eff }}$ we remark: (1) the A1 version $\left(X_{3}=0, X_{s}=0\right)$ is similar to ESC08c; (2) the B1 version $\left(X_{3}=0, X_{s} \neq 0\right)$ is similar to the HALQCD potential [15]. Its $\Xi N\left({ }^{3} S_{1}, T=1\right)$ attraction gives no 'deuteron-like' bound state; (3) A2 and B2 include threebody terms $\left(X_{3} \neq 0\right)$, giving larger separations of the $1 S$ and $2 P$ states. If $V_{\text {eff }}$ has only a three-body term $\left(X_{2}=\right.$ $\left.X_{s}=0\right)$ the $1 S-2 P$ splittings are too large. Thus, we can consider ESC16 $\oplus V_{\text {eff }}$ as an improved versions of ESC08c, where the former models can reproduce the data more nicely than the latter. (In Sec. I for future reference, the models A1, etc., are called ESC16A1, ESC16A2, ESC16B1, and ESC16B2.)

As mentioned already, the $G$-matrix calculations show that the $\Xi^{-}$nucleus interactions the ESC08 and ESC16 models have shortcomings in the $S=-2$ sector, and need to be supplemented by phenomenological interactions. In Sec. VIII an SU(3)-symmetric generalization is given with good results for $N N \oplus Y N$ scattering and well-depths $U_{N}, U_{\Lambda}, U_{\Sigma}$, and $U_{\Xi}$.

\section{ESC16 ${ }^{\star}$ : ESC16 $\oplus$ NEW TWO-BODY FORCES}

As noted in the previous section the observation of the twin $\Lambda$ hypernuclei $[58,59]$ and the recent KISO event [55] are strong indications that the $\Xi$-nucleus interaction is not repulsive, but (moderately) attractive. Two events with "twin" $\Lambda$ hypernuclei (I) [58] and (II) [59] were observed in the KEK 
TABLE XVII. Calculated quantities in $\Xi^{-}+{ }^{12} \mathrm{C}, \Xi^{-}+{ }^{14} \mathrm{~N}, \Xi^{-}+{ }^{16} \mathrm{O}, \Xi^{-}+{ }^{27} \mathrm{Al}$ systems for A1, A2, B1, and B2. Binding energies $B_{\Xi^{-}}$ and conversion width $\Gamma_{\Xi^{-}}^{c}$ are in MeV. rms radii $\sqrt{\left\langle r^{2}\right\rangle}$ are in fm. Averaged Fermi momentum $\bar{k}_{F}$ is in fm ${ }^{-1}$. Coulomb-assisted bound states are marked by $(*)$. The experimental values $B_{\Xi^{-}}(\exp )$ are in $\mathrm{MeV}$.

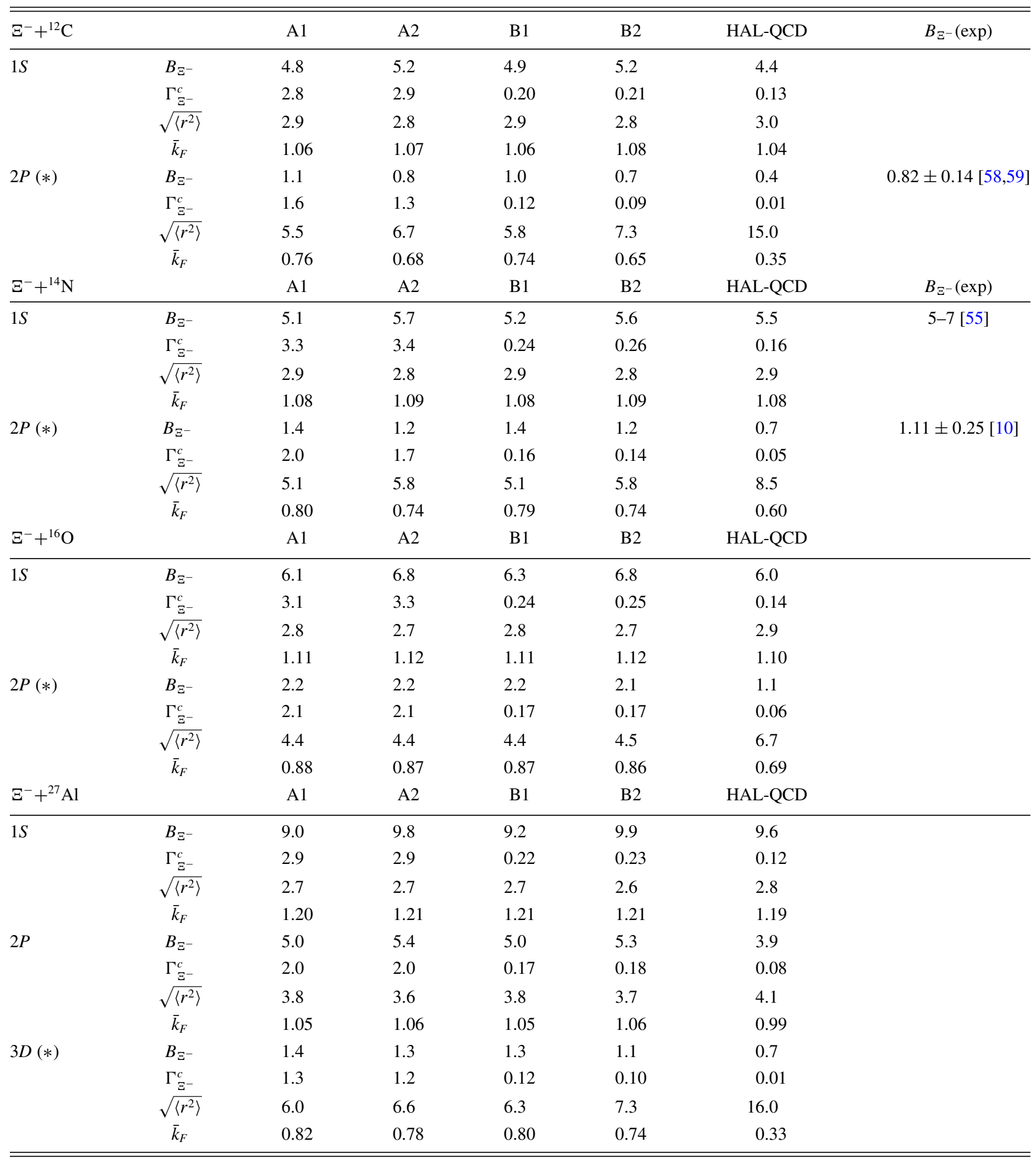

E-176 experiment, and recently a new event (III) [55] has been observed in the KEK E373 experiment. This is in line with the result of the BNL-E885 experiment [19] yielding $U_{\Xi} \approx-14$ $\mathrm{MeV}$. From the results shown in this paper it is clear this can not be accommodated by the two-body forces of the present ESC16 model. Moreover, ESC16 fails to describe the $\Xi^{-} p$ correlations found in the ALICE-experiment at CERN [62]. This situation is also not altered by the inclusion of the 
TABLE XVIII. The $s t$ - and $s u$-crossing matrix $X^{( \pm)}=\left[X_{s t} \pm\right.$ $\left.X_{s u}\right] / 2$. Here, $( \pm)$ refers to the symmetric $\left(27,8_{s}, 1\right)$ and the asymmetric $\left(8_{s}, 10,10^{*}\right)$ irreps, respectively.

\begin{tabular}{cccccccc}
\hline \hline & \multicolumn{7}{c}{$\mu_{\beta^{\prime} \gamma^{\prime}}^{\prime}(t, u)$} \\
\cline { 3 - 8 } & & 27 & $8_{s}$ & 1 & $8_{a}$ & $10^{*}$ & 10 \\
\hline \multirow{4}{*}{$\mu_{\beta \gamma}(s)$} & 27 & $7 / 40$ & $1 / 5$ & $1 / 8$ & - & - & - \\
& $8_{s}$ & $27 / 40$ & $-3 / 10$ & $1 / 8$ & - & - & - \\
& 1 & $27 / 8$ & 1 & $1 / 8$ & - & - & - \\
& $8_{a}(s)$ & - & - & - & $1 / 2$ & 0 & 0 \\
& 10 & - & - & - & 0 & $1 / 4$ & $1 / 4$ \\
& $10^{*}$ & - & - & - & 0 & $1 / 4$ & $1 / 4$ \\
\hline \hline
\end{tabular}

three-body forces produced by the meson-pair interactions. Therefore, there are indications that the two-body forces of the ESC models, although very successful for the $N N$ and $Y N(S=-1)$ interactions, are inadequate for the $\Xi N$ interaction showing its "incompleteness". It is not difficult to suggest possible sources of "incompleteness". For example, in the two-meson exchange potentials only the PS-PS are included, but not PS-VC, PS-SC, VC-VC, VC-SC, etc. Also, the SU(3) structure of the 33 resonances, like the Fujita-Miyazawa interaction, cannot be covered completely by the meson-pair exchanges.

In this section we extend $\mathrm{ESC} 16$ to $\mathrm{ESC} 16^{\star}$ by introducing new SU3-symmetric ( $s$-wave) two-body exchange forces: gaussian central, spin-spin-, and tensor potentials. This in the $t$ and $u$ channels [41] SU(3) irreps $\left\{\mu^{\prime}\right\}:\{27\},\left\{8_{s}\right\},\left\{8_{a}\right\}$, $\left\{10^{*}\right\},\{10\}$. Henceforth, these new potentials, indicated by the subscript $X$, in the $t$ and $u$ channels are denoted as $\widetilde{W}_{c}, \widetilde{W}_{\sigma}$, and $\widetilde{W}_{t}$ for, respectively, the central, spin-spin-, and tensor potentials, and taken of the form

$$
\begin{aligned}
\widetilde{W}_{\mu^{\prime}, c}(r) & =C_{\left\{\mu^{\prime}\right\}} f_{W}(r), \\
\widetilde{W}_{\mu^{\prime}, \sigma}(r) & =S_{\left\{\mu^{\prime}\right\}} f_{W}(r), \\
\widetilde{W}_{\mu^{\prime}, t}(r) & =-(4 / 3) T_{\left\{\mu^{\prime}\right\}}\left(m_{W} r\right)^{2} f_{W}(r),
\end{aligned}
$$

where $C, S, T$ are the $t$ - and $u$-channel coefficients for the central (C), spin-spin (S), and tensor (T) potentials, and $f_{W}(r)=$ $\exp \left(-m_{W}^{2} r^{2}\right), m_{W}=300 \mathrm{MeV}$. For simplicity a universal profile is assumed, which is similar to that of the Pomeron. No specific space-time structure is imposed leaving the central, spin-spin, and tensor unrelated.

In general, the potentials in the $s$-channel irreps are related to those in the $t$ and $u$ channel by the crossing matrices $X_{s t}, X_{s u}$. For the so-called signatured potentials $V^{( \pm)}=$ $[V(t) \pm V(u)] / 2$ these can be combined. In Table XVIII the $\mathrm{SU}(3)$ crossing matrices $X^{( \pm)}$, derived from Ref. [63], for the signatured potentials are given. The SU(3)-singlet exchange gives no distinction for the $s$-channel irreps and therefore is not included in the new SU(3)-symmetric potentials.

The $s$-channel potentials in the $\{\mu\}$-irrep corresponding to Eq. (8.1) are

$$
W_{\mu, c}^{( \pm)}(r)=\sum_{\mu^{\prime}} X^{( \pm)}\left(\mu, \mu^{\prime}\right) C_{\left\{\mu^{\prime}\right\}} f_{W}(r),
$$

TABLE XIX. ESC16 ${ }^{\star}(\mathrm{A})$ : Coupling constants SU(3)-symmetric gaussian potentials.

\begin{tabular}{lccccc}
\hline \hline$\{\mu\}$ & $\{27\}$ & $\left\{8_{s}\right\}$ & $\left\{8_{a}\right\}$ & $\left\{10^{*}\right\}$ & $\{10\}$ \\
\hline $\mathrm{C}_{\{\mu\}}$ & -0.41 & -0.19 & -6.64 & +0.12 & -2.66 \\
$\mathrm{~S}_{\{\mu\}}$ & -0.13 & +0.90 & +7.19 & +0.49 & 0.0 \\
$\mathrm{~T}_{\{\mu\}}$ & - & - & - & - & - \\
\hline \hline
\end{tabular}

and similarly for the spin-spin and tensor potentials. Finally, the two-particle $s$-channel potentials are obtained using the transformations given in Appendix C.

Below we present results of $N N \oplus Y N$ fits having the same quality as for ESC16, with these new SU(3)-symmetric phenomenological potentials for two choices of the extra parameters. In both cases, the meson coupling constants are about equal to those obtained with ESC16, which is also the case for the MPE couplings. It appears that the QPC pattern of the baryon-baryon-meson $g_{B B M}$ couplings is preserved.

The well-depths $U_{N N}, U_{\Lambda}, U_{\Sigma}, U_{\Xi}$ are calculated with the inclusion of the three-body forces. The latter in the form of effective two-body forces from: (a) meson-pair interactions (MPE3), (b) multi-Pomeron (MPP3) with the tripleand quartic-Pomeron couplings $g_{3 P}=2.0, g_{4 P}=20.0$, and (c) Fuji-Miyazawa three-body force (FM3). The gaussian cut-off's employed for the meson-pair vertices are $\Lambda_{p r}$ and $\Lambda_{F M}$ for MPP3 and FM3, respectively. We found that $\Lambda_{p r}=$ $450 \mathrm{MeV}$ gives good results, and used $\Lambda_{F M}$ to tune the nuclear well depth to its experimental value. In Appendix B a list of the used TBF potentials is given. For ESC16 $6^{\star}$ the $N N \oplus Y N$ fit has $\chi_{N N}^{2}(p d p)=1.09, \chi_{Y N}^{2}(p d p)=0.95$, which is of the same quality as that of ESC16. The meson coupling constants of ESC16* are about equal to those obtained with ESC16, which is also the case for the MPE couplings.

At present, experimentally it is not clear from which states the major attractive contribution to $U_{\Xi}$ comes. Therefore, we present two possible solutions: $\operatorname{ESC}^{\star} 6^{\star}(\mathrm{A})$ and $\operatorname{ESC}_{1} 6^{\star}(\mathrm{B})$. In these applications we exploit only central and spin-spin extra gaussian potentials.

$\operatorname{ESCl}^{\star}(A)$ : In this case we put $S_{\{10\}}=0$. The central and spin-spin parameters of the new potentials in $\mathrm{ESC}^{\star} 6^{\star}$ from the fit are listed in Table XIX. No new tensor potentials were included. A comparison of Tables XX and XIII shows that the

TABLE XX. ESC16 ${ }^{\star}(\mathrm{A}): L=0,1,2$ partial-wave total cross

\begin{tabular}{|c|c|c|c|c|c|c|c|c|c|}
\hline \multirow[b]{2}{*}{$\mathrm{L}$} & \multicolumn{3}{|c|}{$\Xi^{-} p \rightarrow \Lambda \Lambda$} & \multicolumn{3}{|c|}{$\Xi^{-} p \rightarrow \Xi^{0} n$} & \multicolumn{3}{|c|}{$\Xi^{-} p \rightarrow \Xi^{-} p$} \\
\hline & $\sigma_{s}$ & $\sigma_{t}$ & $\sigma_{T}$ & $\sigma_{s}$ & $\sigma_{t}$ & $\sigma_{T}$ & $\sigma_{s}$ & $\sigma_{t}$ & $\sigma_{T}$ \\
\hline 0 & 1.99 & - & 1.99 & 3.32 & 2.56 & 5.88 & 8.13 & 1.14 & 9.27 \\
\hline 1 & - & 0.55 & 0.55 & 0.27 & 1.72 & 1.99 & 1.22 & 0.78 & 2.00 \\
\hline 2 & 0.04 & - & 0.04 & 0.08 & 0.38 & 0.46 & 0.05 & 0.10 & 0.15 \\
\hline Tot & 1.93 & 0.55 & 2.58 & 3.67 & 4.66 & 8.33 & 9.40 & 2.02 & 11.22 \\
\hline Exp & & $4.3_{-2.7}^{+6.3}$ & & & $\leqslant 10$ & & & $\leqslant 24$ & \\
\hline
\end{tabular}
sections $\Xi^{-} p \rightarrow \Lambda \Lambda, \Xi^{0} n, \Xi^{-} p, \Sigma^{0} \Lambda$ in $(\mathrm{mb})$ as a function of the beam particle laboratory beam particle momentum $p_{\text {lab }}=500$ $\mathrm{MeV} / c . \sigma_{s}$ and $\sigma_{t}$ are the spin-singlet and spin-triplet cross sections. 
TABLE XXI. ESC16 $6^{\star}(\mathrm{A})$ : The nuclear saturation energy $E_{N}=$ $B / A$, the well depths $U_{\Lambda}, U_{\Sigma}$, and $U_{\Xi}$ with $\mathrm{SU}(3)$-symmetric interactions and three-body forces (TBF) at $k_{F}=1.35 \mathrm{fm}$, and $\Lambda_{F M}=$ $2500 \mathrm{MeV} . E_{N}=T_{N}+U_{N}$ with $T_{N}=22.626 \mathrm{MeV}$.

\begin{tabular}{lcccc}
\hline \hline & $E_{N}$ & $U_{\Lambda}$ & $U_{\Sigma}$ & $U_{\Xi}$ \\
\hline "exp" & -16.3 & -37.9 & +9.9 & -14.0 \\
ESC16 & -19.9 & -48.9 & -26.1 & -36.5 \\
\& TBF & -16.4 & -36.7 & +8.0 & -17.8 \\
\hline \hline
\end{tabular}

cross sections are for $p_{\text {lab }}=500 \mathrm{MeV} / c$ not much different. As can be seen in Table XXI the well depths for ESC16* are much improved compared to ESC16. In this table the "exp" values are the experimental nuclear saturation point $E_{N}$, the $U_{\Lambda}$ and $U_{\Sigma}$ well depth's from paper II [2], and for $U_{\Xi}$ the [19] datum. The partial wave contributions to the $U_{\Xi}$ well depth are displayed in Table XXII. Here, ESC $16^{\star+}=\mathrm{ESC}^{\star} 6^{\star}$ and TBF. The attraction for $U_{\Xi}$ comes from the $T=0$ states, in particular the $\Xi N\left({ }^{1} S_{0}, T=0\right)$ and the $\Xi N\left({ }^{3} S_{1}, T=0\right)$ state. The $T=1$ states give repulsion which comes mainly from the $\Xi N\left({ }^{1} S_{0}, T=1\right)$ state. (With also a tensor $W_{\mu, t}^{( \pm)} \neq 0$ more attraction in $T=1$ can be generated.)

In the $\mathrm{ESC} 16^{\star}$ model there is enough flexibility to tune $U_{\Xi}$ to a smaller value by refitting, eventually with an extended combined fit including next to the $N N \oplus Y N$ data also the well depth's treated as pseudodata.

$\operatorname{ESC} 6^{\star}(B)$ : In this case we have imposed $R$-conjugation symmetry [64], which implies that $C_{10}=C_{10^{*}}, S_{10}=S_{10^{*}}$, and $T_{10}=T_{10^{*}}$. [ $R$ conjugation is not an $\mathrm{SU}(3)$-symmetry, but an approximate symmetry of the ESC-model. It forbids, e.g., the ${ }^{3} P_{1} \leftrightarrow{ }^{1} P_{1}$ transition, which is allowed under SU(3).] The results for this model are presented in Tables XXIII, XXIV, and XXV. A comparison of Tables XXII and XXV shows that the major attractive contribution comes from the $\left({ }^{1} S_{0}, T=0\right)$ and $\left({ }^{3} S_{1}, T=0\right)$ partial wave for $\mathrm{ESC}_{16}{ }^{\star}(\mathrm{A})$ and $\operatorname{ESC} 16^{\star}(\mathrm{B})$, respectively. These results show that the extra freedom generated by the introduction of the $\mathrm{SU}(3)$-symmetric new gaussian potentials $W_{\mu, v}^{( \pm)}(r),(v=$ $c, s, t$ ) leads to a $\mathrm{BB}$ interaction which is useful in providing a good basis for calculations in nuclear and hypernuclear physics.

TABLE XXII. ESC16 ${ }^{\star}(\mathrm{A})$ : Partial wave contributions to $U_{\Xi}\left(\rho_{0}\right)$ at normal density.

\begin{tabular}{llrrrrrrrr}
\hline \hline model & & ${ }^{1} S_{0}$ & ${ }^{3} S_{1}$ & ${ }^{1} P_{1}$ & ${ }^{3} P_{0}$ & ${ }^{3} P_{1}$ & ${ }^{3} P_{2}$ & $U_{\Xi}$ & $\Gamma_{\Xi}^{c}$ \\
\hline ESC16 & $T=0$ & 2.1 & -0.4 & -0.2 & -5.3 & 1.5 & -1.2 & & \\
& $T=1$ & 9.2 & 7.6 & 1.0 & 0.8 & -2.0 & -0.5 & +13.7 & 5.1 \\
ESC16 $^{\star}$ & $T=0$ & -23.5 & -5.7 & 0.9 & -2.6 & 1.3 & -1.6 & & \\
& $T=1$ & 7.0 & -9.0 & 1.3 & 0.3 & -3.2 & -1.6 & -36.5 & 9.0 \\
ESC16 $^{\star+}$ & $T=0$ & -16.0 & -4.7 & 0.8 & -3.2 & 2.1 & -1.5 & & \\
& $T=1$ & 10.3 & -6.4 & 2.0 & 1.4 & -3.2 & 0.7 & -17.8 & 4.7 \\
\hline \hline
\end{tabular}

TABLE XXIII. ESC16 ${ }^{\star}(B)$ : Coupling constants SU(3)symmetric gaussian potentials.

\begin{tabular}{lccccc}
\hline \hline$\{\mu\}$ & $\{27\}$ & $\left\{8_{s}\right\}$ & $\left\{8_{a}\right\}$ & $\left\{10^{*}\right\}$ & $\{10\}$ \\
\hline $\mathrm{C}_{\{\mu\}}$ & -0.11 & -0.49 & -6.01 & -3.27 & -3.27 \\
$\mathrm{~S}_{\{\mu\}}$ & -0.49 & +1.74 & +6.54 & -0.60 & -0.60 \\
$\mathrm{~T}_{\{\mu\}}$ & - & - & - & - & - \\
\hline \hline
\end{tabular}

\section{SUMMARY AND CONCLUSION}

The strangeness $S=-2$ ESC16 potentials and results presented in this paper are an important step in completing the baryon-baryon interactions for scattering and hypernuclei in the context of broken SU(3)-symmetry using. Apart from the gaussian repulsion from the Pomeron and inclusion of a systematic quark-core effects for all baryon-baryon channels, the major part of the BB interactions are generalized Yukawa potentials from single and double meson exchange. These potentials contain (i) one-boson exchanges, where the coupling constants at the baryon-baryon-meson vertices are restricted by the broken SU(3) symmetry, (ii) two-pseudoscalar exchanges, (iii) meson-pair exchanges. Each type of meson exchange (pseudoscalar, vector, axial-vector, scalar) contains five free parameters: a singlet coupling constant, an octet coupling constant, the $F /(F+D)$ ratio $\alpha$, a meson-mixing angle. The potentials are regularized with gaussian cut-off parameters, which provide a few additional free parameters. As shown in papers I and II the $F /(F+D)$ parameters could be restricted, both for OBE and MPE, by the quark-model predictions in the form of the ${ }^{3} P_{0}$ quark-antiquark creation mechanism.

Although we performed truly simultaneous fits to the $N N$ and $Y N$ scattering data, effectively most of these parameters are determined in fitting the rich and accurate $N N$ scattering data, while the remaining ones are fixed by fitting also the (few) $Y N$ scattering data. This still leaves enough flexibility to accommodate the imposition of a few extra constraints. the assumption of SU(3) symmetry for the couplings then allows us to extend these models to the higher strangeness channels (i.e., $Y Y$ with $S=-3,-4$, without the need to introduce additional free parameters. Like the NSC97 models, the ESC04, ESC08, and ESC16 models are very powerful models of this kind, and the very first realistic ones.

Although the different ESC models ESC04, ESC08, and ESC16 produce the $N N$ and $Y N$ data well, there are con-

TABLE XXIV. ESC16 $6^{\star}(\mathrm{B})$ : The nuclear saturation energy $E_{N}=$ $B / A$, the well depths $U_{\Lambda}, U_{\Sigma}$, and $U_{\Xi}$ with $\mathrm{SU}(3)$-symmetric interactions and three-body forces (TBF) at $k_{F}=1.35 \mathrm{fm}$, and $\Lambda_{F M}=$ $1750 \mathrm{MeV}$. $E_{N}=T_{N}+U_{N}$, with $T_{N}=22.626 \mathrm{MeV}$.

\begin{tabular}{lcccr}
\hline \hline & $E_{N}$ & $U_{\Lambda}$ & $U_{\Sigma}$ & $U_{\Xi}$ \\
\hline "exp" & -16.3 & -37.9 & +9.9 & -14.0 \\
ESC16" & -20.2 & -51.5 & -27.3 & -25.2 \\
\& TBF & -14.4 & -32.8 & +17.6 & -9.1 \\
\hline \hline
\end{tabular}


TABLE XXV. ESC16*(B): Partial wave contributions to $U_{\Xi}\left(\rho_{0}\right)$ at normal density.

\begin{tabular}{|c|c|c|c|c|c|c|c|c|c|}
\hline model & & ${ }^{1} S_{0}$ & ${ }^{3} S_{1}$ & ${ }^{1} P_{1}$ & ${ }^{3} P_{0}$ & ${ }^{3} P_{1}$ & ${ }^{3} P_{2}$ & $U_{\Xi}$ & $\Gamma_{\Xi}^{c}$ \\
\hline \multirow[t]{2}{*}{ ESC16 } & $T=0$ & 2.1 & -0.4 & -0.2 & -5.3 & 1.5 & -1.2 & & \\
\hline & $T=1$ & 9.2 & 7.6 & 1.0 & 0.8 & -2.0 & -0.5 & +13.7 & 5.1 \\
\hline ESC16* & $T=1$ & 4.0 & -11.7 & 0.9 & 0.2 & -3.6 & -2.3 & -25.2 & 2.5 \\
\hline \multirow[t]{2}{*}{$\mathrm{ESC} 16^{\star+}$} & $T=0$ & 0.4 & -9.6 & 0.4 & -4.9 & 2.9 & -2.2 & & \\
\hline & $T=1$ & 7.9 & -4.3 & 1.9 & 1.8 & -3.9 & 0.6 & -9.1 & 3.1 \\
\hline
\end{tabular}

siderable differences. In the $N N$ sector the quality of the fit to the $N N$ data of the ESC08/ESC16 models is superior to that for the ESC04 models. Also, they lead to notable differences in the hypernuclear structures, especially in $S=-2$ systems. It is important that at least some of the ESC04d, ESC08a,b,c, and ESC16 solutions predict the well depths, consistently with the indication given by the BNLE885 experiment, and the existence of recently observed $\Xi$ hypernuclei. The $\Xi$-nucleus attraction in ESC08 leads, owing to the extra (phenomenological) contributions from the $(\pi \pi)$ - and $(\pi \omega)$-pair $\Xi N$ interaction, to a substantially attraction in the ${ }^{3} S_{1}\left({ }^{33} S_{1}\right)$ state, with a tensor-potential giving a strong Lane term. This gives a satisfactory value for $U_{\Xi}$, however, also a not observed deuteron-like $D^{*}$ bound state when the extra $(\pi \pi),(\pi \omega)$ pair potentials are interpreted as genuine two-body interactions. (The experimental search for baryon-baryon bound states by the Rome-Saclay-Vanderbilt collaboration [32] in the mass range $2.1-2.5 \mathrm{GeV} / c^{2}$ was negative.)

Recently, most important is the $1 S$-bound states (5-7 $\mathrm{MeV}$ ) and $2 P$-bound states $(1.0-1.5 \mathrm{MeV})$ of the $\Xi^{\mp}{ }^{14} \mathrm{~N}$ system found in emulsions. These data severely select the $\Xi N$ interaction models. The ESC-models, ESC04a-d [5], ESC08ab [6], except ESC08c, seem not good. ESC16 has $U_{\Xi}>0$ and is clearly unsuitable. Then, the only solution in the case ESC16 for the $\Xi N$ interaction is to add a phenomenological (attractive) interaction $V_{\text {eff }}$. Two versions of this additional interaction are worked out: (1) $V_{\text {eff }}=(\pi, \omega)$ pair $\oplus \mathrm{SU}(3)$-singlet potentials, for $S=-2$ only, (2) $V_{\mathrm{eff}}=$ $\mathrm{SU}(3)$-symmetric two-body interactions for all channels, described in Sec. VIII.

Experimentally, the $\Xi N$ interaction seems rather weak as is illustrated by the indication of the $\Xi^{-} p$-scattering data [29-31]. The $\Xi$-nucleus attraction is as indicated by experiments, $U_{\Xi} \approx-14 \mathrm{MeV}$, see Ref. [19] and the recent emulsion-experiments results [10]. These experimental properties are not explained by the ESC16 $\Xi N$ interaction. A possibility is that the three-body force contributes to the well depth, but this is not expected to be large.

To improve the ESC16 model for the $\Xi N$ interaction, while keeping its good features for the $N N$ and $Y N$, and having a sizable $\Xi N$ attraction without a bound state, an extension of the ESC approach is introduced, which is mo- tivated by mentioning possible sources that can lead to a more complete BB interaction. The extension of ESC16 with SU(3)-symmetric phenomenological two-body contact potentials is investigated, and found to be promising for the description of both the $N N, Y N$ scattering as well as the nuclear/hypernuclear well depths. This line can be explored further, both in comparison with other models and applications.

Finally, the conclusion is that the ESC16 potentials are in principle an excellent starting point for calculations and predictions of multistrange systems, but at present need some additional phenomenological potentials in the application to, e.g., $\Xi$-nucleus systems. The nature of these potentials might be two-body as well as three-body. The extension of this work to the $S=-3,4$ systems, i.e., comprising all $\{8\} \otimes\{8\}$ baryon-baryon states, will be the topic of the last paper (IV) in this series.

\section{ACKNOWLEDGMENT}

We thank E. Hiyama, T. Motoba, K. Itonaga, and H.-J. Schulze for many stimulating discussions.

\section{APPENDIX A: CONNECTION PARTICLE AND ISOSPIN BASIS}

The five $S=-2$ channel systems with different charge $q=$ $-2,-1,0,+1,+2$ are listed in Eq. (2.2). The three isospin channels are listed in Eq. (2.3). Using the indices $a, b, c, d$ for

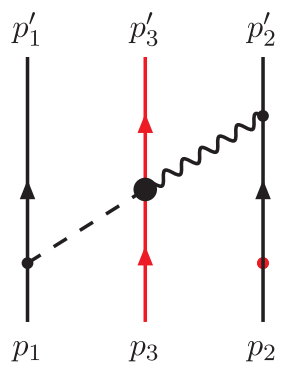

(a)

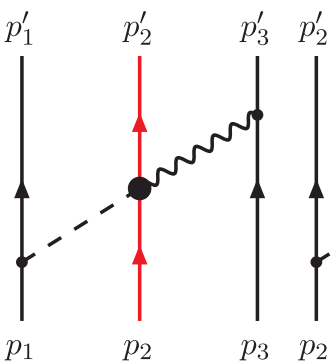

(b)

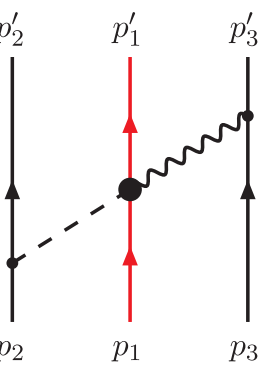

(c)
FIG. 18. Three-body graphs from meson-pair vertices. 
$\Lambda \Lambda, \Xi N, \Sigma \Lambda$, and $\Sigma \Sigma$, respectively, we have [65]

$$
V(q=0)=\left(\begin{array}{cccccc}
V_{a a} & \sqrt{\frac{1}{2}} V_{b a} & -\sqrt{\frac{1}{2}} V_{b a} & 0 & -\sqrt{\frac{1}{3}} V_{a d} & \sqrt{\frac{1}{3}} V_{a d} \\
\cdot & \frac{1}{2}\left[V_{b b}(1)+V_{b b}(0)\right] & \frac{1}{2}\left[V_{b b}(1)-V_{b b}(0)\right] & \sqrt{\frac{1}{2}} V_{b c} & -\sqrt{\frac{1}{6}} V_{b d}(0) & \sqrt{\frac{1}{6}} V_{b d}(0)-\frac{1}{2} V_{b d}(1) \\
\cdot & \frac{1}{2}\left[V_{b b}(1)+V_{b b}(0)\right] & \sqrt{\frac{1}{2}} V_{b c} & \sqrt{\frac{1}{6}} V_{b d}(0) & -\sqrt{\frac{1}{6}} V_{b d}(0)-\frac{1}{2} V_{b d}(1) \\
\cdot & . & V_{c c} & 0 & -\sqrt{\frac{1}{2}} V_{c d} \\
\cdot & . & . & \frac{1}{3}\left[2 V_{d d}(2)+V_{d d}(0)\right] & \frac{1}{3}\left[V_{d d}(2)-V_{d d}(0)\right] \\
\cdot . & . & . & . & \frac{1}{6}\left[V_{d d}(2)+3 V_{d d}(1)+2 V_{d d}(0)\right]
\end{array}\right),
$$

and for $q=+1$ we have

$$
V(q=+1)=\left(\begin{array}{ccc}
V_{b b}(1) & V_{b c} & -\sqrt{\frac{1}{2}} V_{b d} \\
V_{b c} & V_{c c} & -\sqrt{\frac{1}{2}} V_{c d} \\
-\sqrt{\frac{1}{2}} V_{b d}(1) & -\sqrt{\frac{1}{2}} V_{c d} & \frac{1}{2}\left[V_{d d}(1)+V_{d d}(2)\right]
\end{array}\right) .
$$

Here, when necessary an isospin label is added in parentheses.

\section{APPENDIX B: THREE-BODY FORCES AND EFFECTIVE TWO-BODY POTENTIALS}

The included three-body forces in this paper come from (i) meson-pair vertices, (ii) multi-Pomeron vertices, and (iii) decuplet resonances. This is illustrated in Fig. 18. The three-body forces are used in the form of so-called "effective two-body potentials". which are obtained from the full three-body potentials by the application of the LNR approximation [66], which leads to a substantial reduction of the number of contributions from the two-meson-pair vertices. For example, the $(\pi \pi)_{1}$ and $(\pi \rho)_{1}$ potentials vanish completely. In this Appendix we give the reduced form of these MPE potentials after applying the Tr operation: $\operatorname{Tr} \tau_{3}=\operatorname{Tr} \sigma_{3}=0$. In the LNR-procedure the third particle in Fig. 18 is integrated over.

Below the configuration-space effective $N N$ potentials are listed, including only the dominating terms, i.e., we neglect the terms proportional to $1 / M^{2}$.

\section{Meson-pair effective two-body potentials}

$$
\begin{gathered}
J^{P C}=0^{++}: V_{(\pi \pi)_{0}}(r)=-\frac{\left(4 \pi \rho_{N M}\right)}{m_{\pi}^{3}} \frac{g_{(\pi \pi)_{0}}}{4 \pi} \frac{f^{2}}{4 \pi}\left(\boldsymbol{\tau}_{1} \boldsymbol{\tau}_{2}\right)\left(\frac{m_{\pi}}{m_{\pi^{+}}}\right)^{2} m_{\pi}\left[\frac{1}{3} \psi_{C}^{1}\left(m_{\pi}, \frac{\Lambda_{\pi}}{\sqrt{2}}, r\right)\left(\sigma_{1} \boldsymbol{\sigma}_{2}\right)+\psi_{T}^{0}\left(m_{\pi}, \frac{\Lambda_{\pi}}{\sqrt{2}}, r\right) S_{12}\right], \\
J^{P C}=1^{++}: V_{(\pi \sigma)_{1}}^{(1)}(r)=-2 \frac{\left(4 \pi \rho_{N M}\right)}{m_{\pi}^{3}} \frac{g_{(\pi \sigma)_{1}}}{4 \pi} \frac{f_{P} g_{S}}{4 \pi}\left(\boldsymbol{\tau}_{1} \boldsymbol{\tau}_{2}\right)\left(\frac{m_{\pi}^{3}}{m_{\sigma}^{2}}\right)\left[\frac{1}{3} \phi_{C}^{1}\left(m_{\pi}, \Lambda_{\pi}, r\right)\left(\sigma_{1} \boldsymbol{\sigma}_{2}\right)+\phi_{T}^{0}\left(m_{\pi}, \Lambda_{\pi}, r\right) S_{12}\right], \\
V_{(\pi \sigma)_{1}}^{(2)}(r)=-\frac{1}{2} \frac{\left(4 \pi \rho_{N M}\right)}{m_{\pi}^{3}} \frac{g_{(\pi \sigma)_{1}}}{4 \pi} \frac{f_{P} g_{S}}{4 \pi} \frac{m_{\pi}^{2}}{M^{2}}\left(\boldsymbol{\tau}_{1} \boldsymbol{\tau}_{2}\right)\left(\frac{m_{\pi}^{3}}{m_{\sigma}^{2}}\right)\left[\frac{1}{3} \phi_{C}^{2}\left(m_{\pi}, \Lambda_{\pi}, r\right)\left(\sigma_{1} \sigma_{2}\right)+\phi_{T}^{1}\left(m_{\pi}, \Lambda_{\pi}, r\right) S_{12}\right],(\mathrm{B} 1 \mathrm{~B}) \\
J^{P C}=1^{+-}: V_{(\pi \omega)_{1}}^{(2)}(r)=+\frac{\left(4 \pi \rho_{N M}\right)}{m_{\pi}^{3}} \frac{g_{(\pi \omega)_{1}}}{4 \pi} \frac{f_{P} g_{V} \sin \left(\theta_{V}\right)}{4 \pi}\left(\boldsymbol{\tau}_{1} \boldsymbol{\tau}_{2}\right)\left(\frac{m_{\pi}^{3}}{m_{\omega}^{2}}\right)^{-1}\left[\frac{1}{3} \phi_{C}^{1}\left(m_{\pi}, \Lambda_{\pi}, r\right)\left(\sigma_{1} \sigma_{2}\right)+\phi_{T}^{0}\left(m_{\pi}, \Lambda_{\pi}, r\right) S_{12}\right\}, \\
J^{P C}=0^{++}: V_{(\sigma \sigma)}^{(\mathrm{eff})}=-2 \frac{\left(4 \pi \rho_{N M}\right)}{m_{\pi}^{3}} \frac{g_{(\sigma \sigma)}}{4 \pi} \frac{g_{S}^{2}}{4 \pi}\left(\frac{m_{\pi}^{2}}{m_{\sigma}}\right)\left[\phi_{C}^{0}\left(m_{\sigma}, \Lambda_{\sigma}, r\right)-\frac{m_{\sigma}^{2}}{\Lambda_{\sigma}^{2}} \psi_{C}^{0}\left(m_{\sigma}, \Lambda_{\sigma} / \sqrt{2}, r\right)\right] .
\end{gathered}
$$

Here, the function $\psi_{C}^{0}(r, m, \Lambda)=\left(4 \pi / 2 m^{2}\right)(d / d m) I_{2}(m, \Lambda, r), \psi_{C}^{1}=[3 / 2+m(d / d m)] \phi_{C}^{1}(m, \Lambda, r)$, and similarly for $\psi_{T}^{0}$, where $\phi_{C}^{0}(r, m, \Lambda)$, etc., are defined in Ref. [67]. We notice that the pairs of the type $(\pi \pi)_{1}$ and $(\pi \rho)_{1}$ do not contribute to the effective two-body potentials in NN. The SU(3) generalization is similar to the two-body meson-pair potentials described in paper II. 
TABLE XXVI. SU(3) contents of the various potentials on the isospin basis.

\begin{tabular}{|c|c|c|}
\hline \multicolumn{3}{|c|}{ Space-spin antisymmetric states ${ }^{1} S_{0},{ }^{3} P,{ }^{1} D_{2}, \ldots$} \\
\hline$\Lambda \Lambda \rightarrow \Lambda \Lambda$ & $I=0$ & $V_{\Lambda \Lambda, \Lambda \Lambda}=\frac{1}{40}\left(27 V_{27}+8 V_{8_{s}}+5 V_{1}\right)$ \\
\hline$\Lambda \Lambda \rightarrow \Xi N$ & $"$ & $V_{\Lambda \Lambda, \Xi N}=\frac{-1}{40}\left(18 V_{27}-8 V_{8_{s}}-10 V_{1}\right)$ \\
\hline$\Lambda \Lambda \rightarrow \Sigma \Sigma$ & 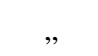 & $V_{\Lambda \Lambda, \Sigma \Sigma}=\frac{\sqrt{3}}{40}\left(-3 V_{27}+8 V_{8_{s}}-5 V_{1}\right)$ \\
\hline$\Xi N \rightarrow \Xi N$ & , & $V_{\Xi N, \Xi N}=\frac{1}{40}\left(12 V_{27}+8 V_{8_{s}}+20 V_{1}\right)$ \\
\hline$\Xi N \rightarrow \Sigma \Sigma$ & " & $V_{\Xi N, \Sigma \Sigma}=\frac{\sqrt{3}}{40}\left(2 V_{27}+8 V_{8_{s}}-10 V_{1}\right)$ \\
\hline$\Sigma \Sigma \rightarrow \Sigma \Sigma$ & , & $V_{\Sigma \Sigma, \Sigma \Sigma}=\frac{1}{40}\left(V_{27}+24 V_{8_{s}}+15 V_{1}\right)$ \\
\hline$\Xi N \rightarrow \Xi N$ & $I=1$ & $V_{\Xi N, \Xi N}=\frac{1}{5}\left(2 V_{27}+3 V_{8_{s}}\right)$ \\
\hline$\Xi N \rightarrow \Lambda \Sigma$ & , & $V_{\Xi N, \Lambda \Sigma}=\frac{\sqrt{6}}{5}\left(V_{27}-V_{8_{s}}\right)$ \\
\hline$\Sigma \Lambda \rightarrow \Sigma \Lambda$ & $"$ & $V_{\Lambda \Sigma, \Lambda \Sigma}=\frac{1}{5}\left(3 V_{27}+2 V_{8_{s}}\right)$ \\
\hline$\Sigma \Sigma \rightarrow \Sigma \Sigma$ & $I=2$ & $V_{\Sigma \Sigma, \Sigma \Sigma}=V_{27}$ \\
\hline
\end{tabular}

\section{Multi-Pomeron effective two-body potentials:}

The "universal", i.e., the same for all elastic BB channels and vanishing for inelastic BB channels, multi-Pomeron (MPP) two-body effective potentials from the triple- and quartic-Pomeron vertices are

$$
\begin{aligned}
& \text { a. } G_{3 P} \text {-vertex : } V_{\mathrm{eff}}(r)=8 g_{3 P} g_{P}^{3} \frac{\rho_{N M}}{\mathcal{M}^{5}} \frac{1}{4 \pi} \frac{4}{\sqrt{\pi}}\left(\frac{m_{P}}{\sqrt{2}}\right)^{3} \exp \left(-\frac{1}{2} m_{P}^{2} r_{12}^{2}\right), \\
& \text { b. } G_{4 P} \text {-vertex : } V_{\mathrm{eff}}(r)=8 g_{4 P} g_{P}^{4} \frac{\rho_{N M}^{2}}{\mathcal{M}^{8}} \frac{4}{\sqrt{\pi}}\left(\frac{m_{P}}{\sqrt{2}}\right)^{3} \exp \left(-\frac{1}{2} m_{P}^{2} r_{12}^{2}\right) .
\end{aligned}
$$

Here, $g_{P}=G_{P} / \sqrt{4 \pi}, g_{3 P}=G_{3 P} /(4 \pi)^{3 / 2}, g_{4 P}=G_{4 P} /(4 \pi)^{2}$, i.e., the rationalized two-, three-, and four-point couplings.

\section{Miyazawa-Fujita effective two-body potentials}

The Hamiltonian for the Fujita-Miyazawa pion-nucleon pair-interaction reads [68]

$$
\mathcal{H}_{F M}=-\bar{\psi}\left[\left\{\left((A+B) \nabla_{\mathbf{1}} \nabla_{\mathbf{2}}+D\right) \delta_{i j}-(A-B) \sigma \nabla_{1} \times \nabla_{2} \epsilon_{i j k} \tau_{k}\right\} \pi_{1, i}(x) \pi_{2, j}(x)\right] \psi .
$$

Here, the spatial derivatives operate on the pion fields, and the constants are

$$
A=\frac{5}{18 \pi} \int \frac{\sigma_{33}}{\omega_{p}^{2}} d p, \quad B=\frac{3}{5} A, \quad D=\frac{2 \pi}{3}\left(a_{1}+2 a_{3}\right)
$$

TABLE XXVII. SU(3) contents of the various potentials on the isospin basis.

\begin{tabular}{lcc}
\hline \hline & Space-spin symmetric states ${ }^{3} S_{1},{ }^{1} P_{1},{ }^{3} D, \ldots$ & \\
\hline$\Xi N \rightarrow \Xi N$ & $I=1$ & $V_{\Xi N, \Xi N}=\frac{1}{3}\left(V_{10}+V_{10^{*}}+V_{8_{a}}\right)$ \\
$\Xi N \rightarrow \Sigma \Lambda$ &, & $V_{\Xi N, \Sigma \Lambda}=\frac{\sqrt{6}}{6}\left(V_{10}-V_{10^{*}}\right)$ \\
$\Xi N \rightarrow \Sigma \Sigma$ &,, & $V_{\Xi N, \Sigma \Sigma}=\frac{\sqrt{2}}{6}\left(V_{10}+V_{10^{*}}-2 V_{8_{a}}\right)$ \\
$\Sigma \Lambda \rightarrow \Sigma \Lambda$ &,, & $V_{\Sigma \Lambda, \Sigma \Lambda}=\frac{1}{2}\left(V_{10}+V_{10^{*}}\right)$ \\
$\Sigma \Lambda \rightarrow \Sigma \Sigma$ &,, & $V_{\Sigma \Lambda, \Sigma \Sigma}=\frac{\sqrt{3}}{6}\left(V_{10}-V_{10^{*}}\right)$ \\
$\Sigma \Sigma \rightarrow \Sigma \Sigma$ &,, & $V_{\Sigma \Sigma, \Sigma \Sigma}=\frac{1}{6}\left(V_{10}+V_{10^{*}}+4 V_{8_{a}}\right)$ \\
$\Xi N \rightarrow \Xi N$ & $I=0$ & $V_{\Xi N, \Xi N}=V_{8_{a}}$ \\
\hline \hline
\end{tabular}


TABLE XXVIII. Pair coupling constants for model ESC16, divided by $\sqrt{4 \pi} . I(M)$ refers to the isospin of the pair $M$ with quantum numbers $J^{P C}$.

\begin{tabular}{|c|c|c|c|c|c|c|c|c|c|c|c|c|}
\hline Pair & $J^{P C}$ & Type & $I(M)$ & $N N M$ & $\Sigma \Sigma M$ & $\Sigma \Lambda M$ & $\Xi \Xi M$ & $I(M)$ & $\Lambda N M$ & $\Lambda \Xi M$ & $\Sigma N M$ & $\Sigma \Xi M$ \\
\hline \multirow[t]{2}{*}{$\pi \eta$} & $0^{++}$ & $g$ & 1 & -0.6881 & -1.3763 & 0.0000 & -0.6881 & $1 / 2$ & 1.1919 & -1.1919 & 0.6881 & 0.6881 \\
\hline & & & 0 & -1.1919 & 0.0000 & 0.0000 & 1.1919 & & & & & \\
\hline & & & 0 & 0.4354 & 0.0000 & 0.0000 & -0.4354 & & & & & \\
\hline \multirow[t]{2}{*}{$\pi \pi$} & $1^{--}$ & $f$ & 1 & -1.7729 & -1.4183 & -1.2283 & 0.3546 & $1 / 2$ & 1.8424 & -0.6141 & -0.3546 & 1.7729 \\
\hline & & & 0 & -0.6141 & 1.2283 & -1.2283 & 1.8424 & & & & & \\
\hline & & & 0 & 1.9715 & -3.9430 & 3.9430 & -5.9145 & & & & & \\
\hline \multirow[t]{2}{*}{$\pi \sigma$} & $1^{++}$ & $g$ & 1 & -0.3892 & -0.3114 & -0.2697 & 0.0778 & $1 / 2$ & 0.4045 & -0.1348 & -0.0778 & 0.3892 \\
\hline & & & 0 & -0.1348 & 0.2697 & -0.2697 & 0.4045 & & & & & \\
\hline \multirow[t]{2}{*}{$\pi \omega$} & $1^{+-}$ & $g$ & 1 & -0.3280 & -0.2624 & -0.2273 & 0.0656 & $1 / 2$ & 0.3409 & -0.1136 & -0.0656 & 0.3280 \\
\hline & & & 0 & -0.1136 & 0.2273 & -0.2273 & 0.3409 & & & & & \\
\hline
\end{tabular}

with the numerical values $\int \sigma_{33} / \omega_{p}^{2} \cdot d p=3.7 m_{\pi}^{-3}$, and $a_{1}+2 a_{3}=-0.06 m_{\pi}^{-1}$. The effective two-body FM-pair $(\pi \pi)_{33^{-}}$ exchange potential is

$$
\begin{aligned}
V_{F M}^{(\text {eff })}= & +2 \rho_{N M}\left(\frac{f_{P}^{2}}{4 \pi}\right) m_{\pi}\left(\boldsymbol{\tau}_{1} \boldsymbol{\tau}_{2}\right)\left[(A+B)\left\{\frac{1}{3} \phi_{C}^{1}\left(m_{\pi}, \Lambda / \sqrt{2}, r_{12}\right)\left(\sigma_{1} \sigma_{2}\right)+\phi_{T}^{0}\left(m_{\pi}, \Lambda / \sqrt{2}, r_{12}\right) S_{12}\right\}\right. \\
& \left.+\frac{2\left((A+B) m_{\pi}^{2}-D\right)}{\Lambda^{2}} \cdot\left\{\frac{1}{3} \psi_{C}^{1}\left(m_{\pi}, \Lambda / \sqrt{2}, r_{12}\right)\left(\sigma_{1} \sigma_{2}\right)+\psi_{T}^{0}\left(m_{\pi}, \Lambda / \sqrt{2}, r_{12}\right) S_{12}\right\}\right] .
\end{aligned}
$$

Since the $D$ term comes from the subtraction constant in the dispersion relation this term is omitted, $D=0$.

The SU(3) generalization of the FM interaction shows that the effects of the decuplet resonances are exchanges in the $t$ channel irreps $\{27\},\left\{8_{s}\right\}$, and $\{1\}$.

The details of the three-body meson pair, multi-Pomeron, and FM potentials can be found in Refs. [69-71], respectively.

\section{APPENDIX C: BARYON-BARYON CHANNELS AND SU(3) IRREPS}

In Tables XXVI and XXVII we give the relation between the potentials on the isospin basis and the potentials in the SU(3) irreps.

\section{APPENDIX D: MESON-PAIR COUPLING CONSTANTS}

In Table XXVIII we give the MPE couplings for model ESC16.

\begin{tabular}{|c|c|c|c|c|c|c|c|c|c|}
\hline$p_{\Lambda}$ & $\delta\left({ }^{1} S_{0}\right)$ & $\rho\left({ }^{1} S_{0}\right)$ & $\delta\left({ }^{3} P_{0}\right)$ & $\rho\left({ }^{3} P_{0}\right)$ & $\delta\left({ }^{3} P_{1}\right)$ & $\rho\left({ }^{3} P_{1}\right)$ & $\delta\left({ }^{3} P_{2}\right)$ & $\epsilon_{2}$ & $\delta\left({ }^{3} F_{2}\right)$ \\
\hline 10 & 0.64 & - & 0.00 & - & 0.00 & - & 0.00 & 0.00 & 0.00 \\
\hline 50 & 3.08 & - & 0.13 & - & 0.01 & - & 0.01 & 0.00 & 0.00 \\
\hline 200 & 7.80 & - & 31.72 & - & 0.26 & - & 0.59 & 0.01 & 0.00 \\
\hline 300 & 7.61 & - & 128.51 & - & 0.36 & - & 1.32 & 0.04 & 0.02 \\
\hline 350 & 10.49 & 12.72 & 106.23 & 3.41 & 0.25 & 0.22 & 1.64 & 0.08 & 0.05 \\
\hline 500 & -4.90 & 21.46 & -15.08 & 19.26 & -1.05 & 3.37 & 1.95 & 0.35 & 0.30 \\
\hline 600 & -13.71 & 21.89 & -17.77 & 21.89 & -2.72 & 5.16 & 1.51 & 0.71 & 0.64 \\
\hline 700 & -22.02 & 21.75 & -21.01 & 23.28 & -4.84 & 6.60 & 0.66 & 1.23 & 1.18 \\
\hline 800 & -29.82 & 21.36 & -24.53 & 23.93 & -7.13 & 7.73 & -0.48 & 1.98 & 2.03 \\
\hline 900 & -37.00 & 20.82 & -28.30 & 24.02 & -8.85 & 8.82 & -1.76 & 3.18 & 3.57 \\
\hline 1000 & -43.83 & 20.68 & -32.01 & 23.07 & -11.42 & 15.84 & -3.47 & 4.84 & 4.22 \\
\hline
\end{tabular}

TABLE XXIX. ESC08c $\Lambda \Lambda \rightarrow \Lambda \Lambda \mathrm{BKS}$-phase parameters in degrees as a function of the laboratory momentum $p_{\Lambda}$ in $\mathrm{MeV} / c$. 


\section{APPENDIX E: BKS-PHASE PARAMETERS}

In Tables XXIX-XXXI we display the BKS-phase parameters for model ESC08c.

TABLE XXX. ESC08c ${ }^{1} S_{0},{ }^{1} P_{1}(\Xi N \rightarrow \Xi N, I=0)$ BKS-phase parameters in degrees as a function of the laboratory momentum $p_{\Lambda}$ in $\mathrm{MeV} / c$.

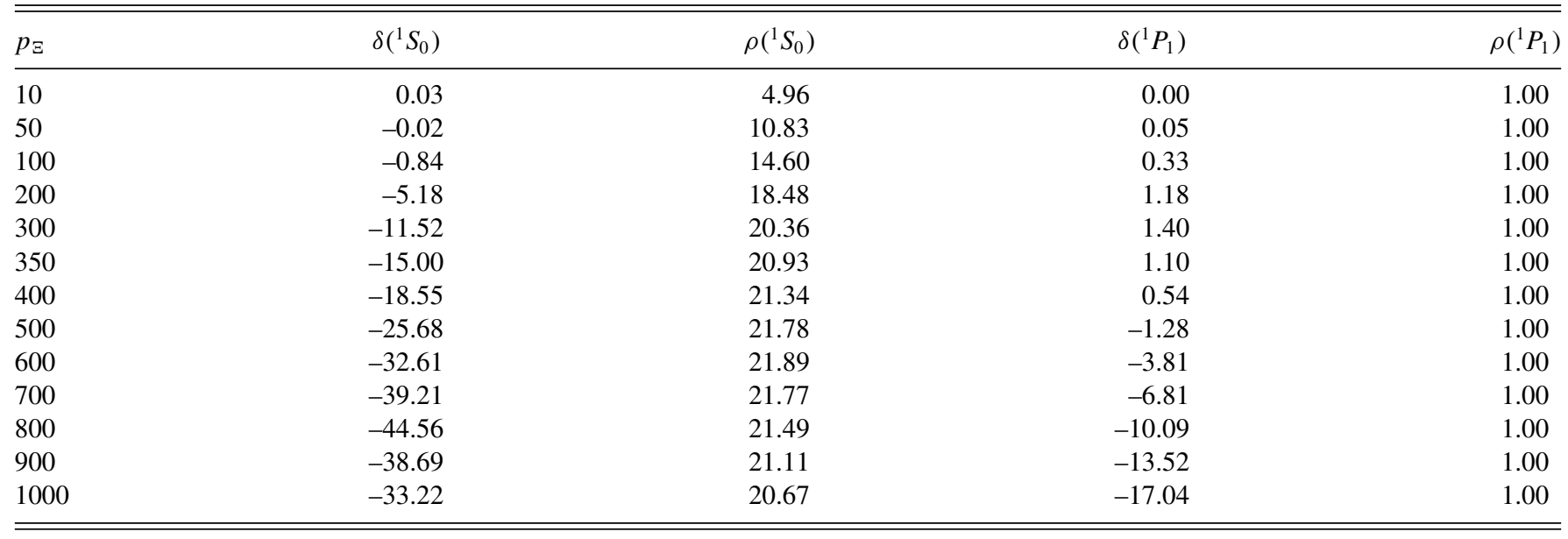

TABLE XXXI. ESC $16{ }^{1} S_{0}(\Xi N \rightarrow \Xi N, I=1)$, etc. BKS-phase parameters in degrees as a function of the laboratory momentum $p_{\Xi}$ in $\mathrm{MeV} / c$.

\begin{tabular}{|c|c|c|c|c|c|c|c|c|c|}
\hline$p_{\Lambda}$ & $\delta\left({ }^{1} S_{0}\right)$ & $\rho\left({ }^{1} S_{0}\right)$ & $\delta\left({ }^{3} P_{0}\right)$ & $\rho\left({ }^{3} P_{0}\right)$ & $\delta\left({ }^{3} P_{1}\right)$ & $\rho\left({ }^{3} P_{1}\right)$ & $\delta\left({ }^{3} P_{2}\right)$ & $\epsilon_{2}$ & $\delta\left({ }^{3} F_{2}\right)$ \\
\hline 50 & -3.32 & - & -0.05 & - & 0.04 & - & 0.00 & 0.00 & -0.00 \\
\hline 200 & -12.14 & - & -1.54 & - & 1.26 & - & -0.03 & 0.12 & -0.01 \\
\hline 300 & -17.15 & - & -3.02 & - & 2.34 & - & -0.28 & 0.35 & -0.05 \\
\hline 350 & -19.41 & - & -3.74 & - & 2.71 & - & -0.55 & 0.48 & -0.08 \\
\hline 600 & -19.07 & 19.18 & -6.47 & 1.02 & 1.96 & 1.06 & -3.56 & 1.07 & -0.21 \\
\hline 700 & -32.61 & 26.30 & -7.20 & 6.55 & 0.67 & 5.56 & -5.44 & 1.21 & -0.21 \\
\hline 800 & -41.84 & 27.11 & -8.64 & 10.29 & -1.45 & 9.43 & -7.57 & 1.28 & -0.16 \\
\hline 900 & -40.63 & 26.93 & -10.79 & 12.79 & -4.16 & 10.28 & -9.87 & 1.29 & -0.04 \\
\hline 1000 & -34.24 & 26.28 & -13.51 & 14.39 & -7.27 & 11.53 & -12.30 & 1.27 & 0.16 \\
\hline
\end{tabular}

TABLE XXXII. ESC16 ${ }^{1} S_{0},{ }^{3} S_{1}-{ }^{3} D_{1}(\Xi N \rightarrow \Xi N, I=0)$ BKS-phase parameters in degrees as a function of the laboratory momentum $p_{\Xi}$ in $\mathrm{MeV} / c$.

\begin{tabular}{|c|c|c|c|c|c|}
\hline$p_{\Xi}$ & $\delta\left({ }^{1} S_{0}\right)$ & $\rho\left({ }^{1} S_{0}\right)$ & $\delta\left({ }^{3} S_{1}\right)$ & $\epsilon_{1}$ & $\delta\left({ }^{3} D_{1}\right)$ \\
\hline 10 & 0.03 & 4.96 & 0.33 & 0.00 & 0.00 \\
\hline 50 & -0.02 & 10.83 & 1.69 & 0.09 & 0.00 \\
\hline 200 & -5.18 & 18.48 & 6.46 & 2.97 & 0.30 \\
\hline 300 & -11.52 & 20.36 & 7.48 & 5.79 & 0.85 \\
\hline 400 & -18.55 & 21.34 & 6.42 & 8.44 & 1.61 \\
\hline 600 & -32.61 & 21.89 & 0.52 & 13.11 & 4.12 \\
\hline 700 & -39.21 & 21.77 & -3.45 & 15.15 & 6.08 \\
\hline 800 & -44.56 & 21.49 & -7.78 & 16.96 & 8.50 \\
\hline 900 & -38.69 & 21.11 & -12.30 & 18.44 & 11.24 \\
\hline 1000 & -33.22 & 20.67 & -16.81 & 19.49 & 14.02 \\
\hline
\end{tabular}


TABLE XXXIII. ESC16 ${ }^{1} P_{1},{ }^{3} S_{1}-{ }^{3} D_{1}(\Xi N \rightarrow \Xi N, I=1)$ BKS-phase parameters in degrees as a function of the laboratory momentum $p_{\Xi}$ in $\mathrm{MeV} / c$.

\begin{tabular}{lrrrrrrr}
\hline \hline$p_{\Xi}$ & $\delta\left({ }^{1} P_{1}\right)$ & $\rho\left({ }^{1} P_{1}\right)$ & $\delta\left({ }^{3} S_{1}\right)$ & $\epsilon_{1}$ & $\delta\left({ }^{3} D_{1}\right)$ & $\eta_{11}$ & $\eta_{12}$ \\
\hline 10 & -0.00 & 0.00 & -0.15 & 0.00 & -0.00 & 1.00 & 0.00 \\
50 & -0.02 & 0.00 & -0.80 & 0.02 & -0.00 & 1.00 & 0.00 \\
100 & -0.12 & 0.00 & -1.75 & 0.16 & -0.01 & 1.00 & 0.00 \\
200 & -0.54 & 0.00 & -4.32 & 0.69 & -0.08 & 1.00 & 0.00 \\
300 & -1.11 & 0.00 & -7.66 & 1.24 & -0.23 & 1.00 & 0.00 \\
400 & -1.91 & 0.00 & -11.44 & 1.65 & -0.36 & 1.00 & -0.01 \\
500 & -2.97 & 0.00 & -15.26 & 1.94 & -0.36 & 1.00 & -0.02 \\
600 & -3.97 & 1.47 & -17.58 & 2.66 & -0.02 & 0.95 & -0.03 \\
700 & -5.08 & 8.01 & -24.26 & 2.10 & 0.06 & 0.90 & -0.03 \\
800 & -7.20 & 12.12 & -29.73 & 1.68 & 0.47 & 0.91 & -0.00 \\
900 & -10.12 & 14.83 & -34.61 & 1.45 & 1.41 & 0.92 & -0.03 \\
1000 & -13.58 & 16.48 & -38.96 & 1.45 & 2.28 & 0.93 & -0.93 \\
\hline \hline
\end{tabular}

TABLE XXXIV. ESC $16{ }^{1} S_{0},{ }^{3} S_{1}-{ }^{3} D_{1}(\Sigma \Lambda \rightarrow \Sigma \Lambda, I=1)$ BKS-phase parameters in degrees as a function of the laboratory momentum $p_{\Xi}$ in $\mathrm{MeV} / c$.

\begin{tabular}{lrrrrrrr}
\hline \hline$p_{\Sigma}$ & $\delta\left({ }^{1} S_{0}\right)$ & $\rho\left({ }^{1} S_{0}\right)$ & $\delta\left({ }^{3} S_{1}\right)$ & $\epsilon_{1}$ & $\delta\left({ }^{3} D_{1}\right)$ & $\eta_{11}$ & $\eta_{12}$ \\
\hline 10 & 0.18 & 6.76 & 0.14 & 0.00 & -0.00 & 0.99 & -0.00 \\
50 & 0.70 & 14.60 & 0.61 & 0.08 & -0.00 & 0.97 & -0.00 \\
100 & 0.53 & 19.43 & 0.68 & 0.51 & -0.02 & 0.94 & -0.00 \\
200 & -2.58 & 24.08 & -1.25 & 1.98 & -0.30 & 0.91 & -0.00 \\
300 & -10.90 & 26.10 & -5.28 & 3.39 & -0.84 & 0.89 & -0.01 \\
400 & -14.09 & 26.95 & -10.35 & 4.50 & -1.33 & 0.88 & -0.03 \\
500 & -20.64 & 27.11 & -15.73 & 5.42 & -1.46 & 0.88 & -0.06 \\
600 & -27.17 & 26.78 & -20.93 & 6.57 & -0.27 & 0.88 & -0.09 \\
700 & -33.49 & 26.07 & -27.60 & 6.62 & -2.98 & 0.87 & -0.12 \\
800 & -39.49 & 25.09 & -33.63 & 4.60 & -8.39 & 0.89 & 0.99 \\
900 & -44.90 & 23.90 & -38.87 & 3.64 & -11.41 & 0.90 & -0.12 \\
1000 & -39.67 & 22.58 & -43.72 & 3.10 & -13.65 & 0.91 & -0.06 \\
\hline \hline
\end{tabular}

TABLE XXXV. ESC16 $I=2, L=0, L=1 \Sigma^{ \pm} \Sigma^{ \pm} \rightarrow \Sigma^{ \pm} \Sigma^{ \pm}$BKS-phase parameters in degrees as a function of the laboratory momentum $p_{\Xi}$ in $\mathrm{MeV} / c$. In parentheses the phases without Coulomb are listed.

\begin{tabular}{|c|c|c|c|c|c|c|}
\hline$p_{\Sigma}$ & $\delta\left({ }^{1} S_{0}\right)$ & $\delta\left({ }^{3} P_{0}\right)$ & $\delta\left({ }^{3} P_{1}\right)$ & $\delta\left({ }^{3} P_{2}\right)$ & $\epsilon_{2}$ & $\delta\left({ }^{3} F_{2}\right)$ \\
\hline 10 & $-14.73(-0.29)$ & $-0.20(0.00)$ & $-0.22(-0.00)$ & $-0.21(0.00)$ & 0.00 & $-0.00(0.00)$ \\
\hline 50 & $-19.07(-1.60)$ & $-3.07(0.20)$ & $-3.43(-0.13)$ & $-3.27(0.01)$ & 0.00 & $-0.01(0.00)$ \\
\hline 200 & $-19.28(-11.40)$ & $0.14(5.33)$ & $-8.97(-3.55)$ & $-4.70(0.75)$ & 0.44 & $-3.25(0.05)$ \\
\hline 300 & $-26.49(-20.53)$ & $3.90(8.22)$ & $-11.35(-7.06)$ & $-2.43(1.87)$ & 1.13 & $-2.68(0.22)$ \\
\hline 400 & $-35.22(-30.36)$ & $4.67(8.27)$ & $-14.57(-10.99)$ & $-0.57(3.01)$ & 1.85 & $-2.06(0.47)$ \\
\hline 600 & $-36.13(-39.76)$ & $0.11(2.86)$ & $-22.37(-19.63)$ & $2.03(4.77)$ & 2.81 & $-1.23(0.79)$ \\
\hline 700 & $-26.90(-30.14)$ & $-3.34(-0.86)$ & $-26.53(-24.06)$ & $2.82(5.28)$ & 3.02 & $-1.24(0.60)$ \\
\hline 800 & $-17.93(-20.86)$ & $-6.92(-4.66)$ & $-30.72(-28.46)$ & $3.15(5.40)$ & 3.09 & $-1.65(0.06)$ \\
\hline 900 & $-9.30(-11.99)$ & $-10.41(-8.35)$ & $-34.87(-32.77)$ & $2.93(5.01)$ & 3.11 & $-2.45(-0.87)$ \\
\hline 1000 & $-1.07(-3.57)$ & $-13.86(-11.89)$ & $-38.96(-36.99)$ & $2.14(4.09)$ & 3.10 & $-3.63(-2.13)$ \\
\hline
\end{tabular}


[1] M. M. Nagels, Th. A. Rijken, and Y. Yamamoto, Phys. Rev. C 99, 044002 (2019).

[2] M. M. Nagels, Th. A. Rijken, and Y. Yamamoto, Phys. Rev. C 99, 044003 (2019).

[3] Th. A. Rijken, Phys. Rev. C 73, 044007 (2006).

[4] Th. A. Rijken and Y. Yamamoto, Phys. Rev. C 73, 044008 (2006).

[5] Th. A. Rijken and Y. Yamamoto, arXiv:nucl-th/060807.

[6] Th. A. Rijken, M. M. Nagels, and Y. Yamamoto, Prog. Theor. Phys. Suppl. 185, 14 (2010).

[7] V. G. J. Stoks and Th. A. Rijken, Phys. Rev. C 59, 3009 (1999).

[8] H. Takahashi et al., Phys. Rev. Lett. 87, 212502 (2001).

[9] K. Nakazawa et al., Nucl. Phys. A 835, 207 (2010).

[10] K. Nakazawa et al., Prog. Theor. Exp. Phys. 2015, 033 D02 (2015).

[11] M. Danysz et al., Nucl. Phys. 49, 121 (1963).

[12] D. J. Prowse, Phys. Rev. Lett. 17, 782 (1966).

[13] K. W. Li, T. Hyodo, and L. S. Geng, Phys. Rev. C 98, 065203 (2018).

[14] J. Haidenbauer and U.-G. Meissner, Eur. Phys. J. A 55, 23 (2019).

[15] K. Sasaki (HAL-QCD Collaboration), Nucl. Phys. A 998, 121737 (2020).

[16] L. Micu, Nucl. Phys. B 10, 521 (1969); A. Le Yaouanc, L. Oliver, O. P'ene, and J. C. Raynal, Phys. Rev. D 8, 2223 (1973); 9, 1415 (1974).

[17] Th. A. Rijken, Baryon-baryon Couplings in the ${ }^{3} P_{0}$ and ${ }^{3} S_{1}$ QPC-models, Notes University of Nijmegen, The Netherlands, nn-online, THEF 12.01.

[18] N. Isgur and J. Paton, Phys. Rev. D 31, 2910 (1985); R. Kokoski and N. Isgur, ibid. 35, 907 (1987); T. J. Burns and F. E. Close, ibid. 74, 034003 (2006).

[19] T. Fukuda et al., Phys. Rev. C 58, 1306 (1998); P. Khaustov et al., ibid. 61, 054603 (2000).

[20] Y. Yamamoto and H. Bandō, Prog. Theor. Phys. 83, 254 (1990).

[21] Y. Yamamoto, T. Motoba, and Th. A. Rijken, Prog. Theor. Phys. Suppl. 185, 72 (2010).

[22] M. M. Nagels, Th. A. Rijken, and Y. Yamamoto, arXiv:nuclth/1504.02634.

[23] Th. A. Rijken, V. G. J. Stoks, and Y. Yamamoto, Phys. Rev. C 59, 21 (1999).

[24] P. M. M. Maessen, Th. A. Rijken, and J. J. de Swart, Phys. Rev. C 40, 2226 (1989).

[25] R. A. Bryan, Phys. Rev. C 24, 2659 (1981); 30, 305 (1984).

[26] S. Klarsfeld, Phys. Lett. B 126, 148 (1983).

[27] D. W. L. Sprung, Phys. Rev. C 32, 699 (1985).

[28] A. Kabir and M. W. Kermode, J. Phys. G: Nucl. Phys. 13, 501 (1987).

[29] J. K. Ahn et al. (KEK-224 Collaboration), Phys. Lett. B 633, 214 (2006).

[30] J. K. Ahn et al. (KEK-224 Collaboration), Nucl. Phys. A 625, 231 (1997).

[31] J. K. Ahn et al., arXiv:0502010v1 [nucl-ex].

[32] D'Agostini et al. (Rome-Saclay-Vanderbilt Collaboration), Nucl. Phys. B 209, 1 (1982).

[33] M. M. Nagels, T. A. Rijken, and J. J. de Swart, Ann. Phys. (NY) 79, 338 (1973).

[34] J. J. de Swart, Rev. Mod. Phys. 35, 916 (1963); 37, 326(E) (1965).

[35] J. J. de Swart, Proceedings of the 1966 CERN SCHOOL OF PHYSICS, CERN 66-29, Vol. II, pp. 1-84, Geneva 1966.
[36] R. H. Thompson, Phys. Rev. D 1, 110 (1970).

[37] Th. A. Rijken, Ann. Phys. (NY) 208, 253 (1991).

[38] Th. A. Rijken and V. G. J. Stoks, Phys. Rev. C 46, 73 (1992); 46, 102 (1992).

[39] Th. A. Rijken and V. G. J. Stoks, Phys. Rev. C 54, 2851 (1996).

[40] Th. A. Rijken and V. G. J. Stoks, Phys. Rev. C 54, 2869 (1996).

[41] S. Mandelstam, Phys. Rev. 112, 1344 (1958).

[42] J. J. de Swart, M. M. Nagels, T. A. Rijken, and P. A. Verhoeven, Springer Tracts Mod. Phys. 60, 138 (1971).

[43] J. J. de Swart and C. K. Iddings, Phys. Rev. 128, 2810 (1962); 130, 319 (1963).

[44] Y. C. Tang and B. C. Herndon, Phys. Rev. 138, B637 (1965).

[45] A. R. Bodmer and S. Ali, Phys. Rev. 138, B644 (1965).

[46] R. H. Dalitz, D. H. Davis, P. H. Fowler, A. Montwill, J. Pniewski, and J. A. Zakrewski, Proc. R. Soc. London A 426, 1 (1989)

[47] A. Ohnishi, K. Morita, K. Miyahara, and T. Hyodo, Nucl. Phys. A 954, 294 (2016).

[48] H. P. Stapp, T. Ypsilantis, and N. Metropolis, Phys. Rev. 105, 302 (1957).

[49] M. Gell-Mann, Phys. Rev. 125, 1067 (1962); S. Okubo, Prog. Theor. Phys. 27, 949 (1962); M. Gell-Mann, ibid. 28, 24 (1962).

[50] T. Inoue, N. Ishii, S. Aoki, T. Doi, T. Hatsuda, Y. Ikeda, K. Murano, H. Nemura, and K. Sasaki, Phys. Rev. Lett. 106, 162002 (2011).

[51] T. Inoue et al., Nucl. Phys. A 881, 28 (2012).

[52] R. L. Jaffe, Phys. Rev. Lett. 38, 195 (1977); 38, 617(E) (1977).

[53] B. H. Kim et al., Phys. Rev. Lett. 110, 222002 (2013).

[54] T. Nagae, Proceedings of APPC14, Kuching, Malaysia, 17-22 November 2019.

[55] K. Nakazawa, private communication.

[56] M. Kohno, Phys. Rev. C 100, 024313 (2019).

[57] Y. Yamamoto, T. Motoba, T. Fukuda, M. Takahashi, and K. Ikeda, Prog. Theor. Phys. Suppl. 117, 281 (1994).

[58] S. Aoki et al., Prog. Theor. Phys. 89, 493 (1993).

[59] S. Aoki et al., Phys. Lett. B 355, 45 (1995).

[60] T. Gogami et al., Phys. Rev. C 93, 034314 (2016).

[61] E. Hiyama, M. Kamimura, Y. Yamamoto, T. Motoba, and Th. A. Rijken, Suppl. Progr. Theor. Phys. 185, 106 (2010); 185, 152 (2010)

[62] L. Fabietti et al. (ALICE Collaboration), Phys. Lett. B 805, 135419 (2020).

[63] J. J. De Swart, Nuovo Cimento 31, 420 (1964).

[64] M. Gell-Mann, California Institute of Technology Laboratory, Report CTSL-20 (1961), reprinted in The Eightfold Way (Benjamin, Inc., Reading, MA, 1964)

[65] P. M. M. Maessen, private communication.

[66] B. A. Loiseau, Y. Nogami, and C. K. Ross, Nucl. Phys. A 165, 601 (1971).

[67] MM. M. Nagels, T. A. Rijken, and J. J. de Swart, Phys. Rev. D 17, 768 (1978); Nucl. Phys. A 285, 429 (1977).

[68] J.-I. Fujita and H. Miyazawa, Prog. Theor. Phys. 17, 360 (1957).

[69] Th. A. Rijken, GESC18: Two-Meson-Pair Exchange YNN Potentials, $\Lambda N, \Sigma N, \Xi N$ G-matrix Application, NN-Online facility: http://nn-online.org

[70] Th. A. Rijken, Multi-pomeron Exchange and the Universal Repulsion in Nuclear/Hyperonic Matter, NN-Online facility: http://nn-online.org

[71] Th. A. Rijken, Derivative Scalar- and Miyazawa-Fujita-Pair Exchange BB-potentials, NN-Online facility: http://nn-online.org 